Supporting Information

\title{
Regiodivergent Photocyclization of Dearomatized
}

\section{Acylphloroglucinols: Asymmetric Syntheses of (-)-Nemorosone and (-)-6-epi-Garcimultiflorone A}

Saishuai Wen, ${ }^{1}$ Jonathan H. Boyce,${ }^{1}$ Sunil K. Kandappa,${ }^{2}$ Jayaraman Sivaguru, ${ }^{2, *}$ and John A. Porco, Jr. ${ }^{1, *}$

${ }^{1}$ Department of Chemistry, Center for Molecular Discovery (BU-CMD), Boston University, 590 Commonwealth Avenue, Boston MA 02215

${ }^{2}$ Center for Photocheimcal Sciences and Department of Chemistry, Bowling Green State University, Bowling Green, OH 43403-0001

*porco@bu.edu andsivagj@bgsu.edu 


\section{TABLE OF CONTENTS}

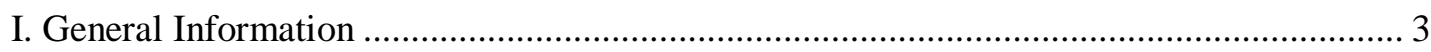

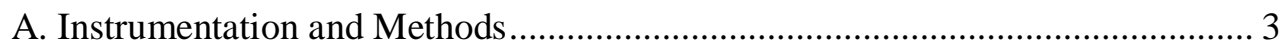

B. Reagents and Solvents ............................................................................ 4

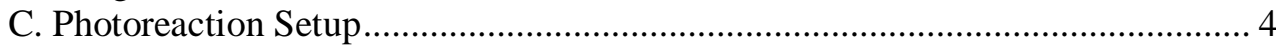

II. Experimental Procedures and Compound Characterization ......................................... 7

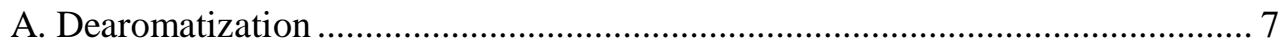

B. Photocyclization of Dearomatized Substrate and Condition Optimization......... 10

C. Confirmation of the Type A Scaffolds ............................................................. 15

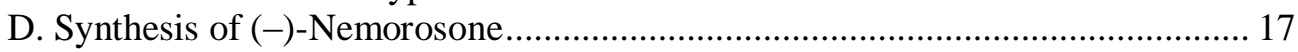

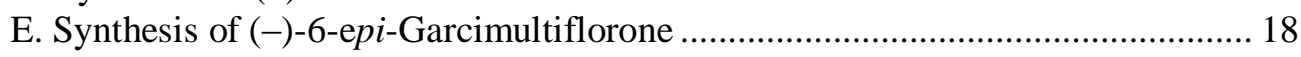

F. Derivatization of Dearomatized Compound 22 ............................................ 20

G. Argon/Oxygen-Bubbling Experiments ……................................................. 21

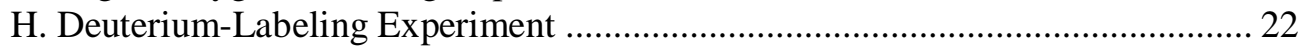

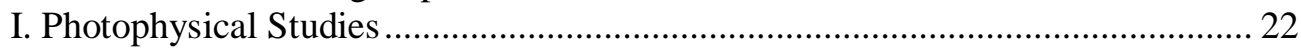

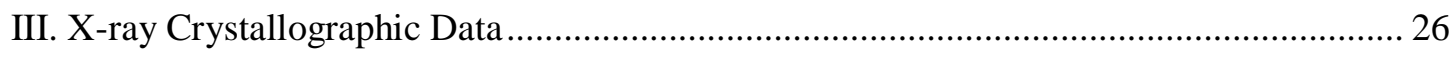

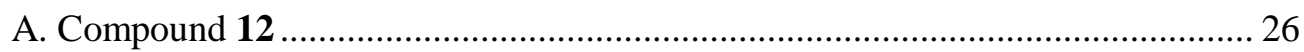

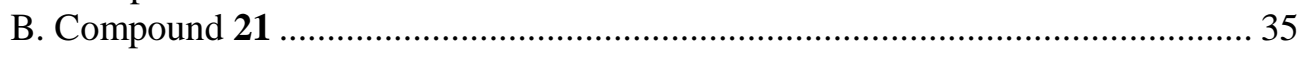

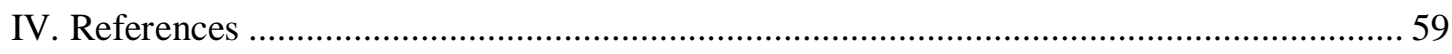

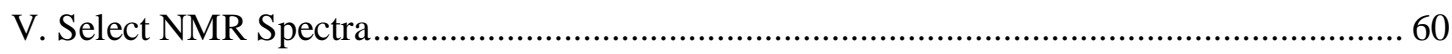




\section{General Information}

\section{A. Instrumentation and Methods}

All NMR spectra were obtained in chloroform- $d\left(\mathrm{CDCl}_{3}\right)$ or methanol- $d_{4}\left(\mathrm{CD}_{3} \mathrm{OD}\right)$ from Cambridge Isotope Laboratories, Inc. at ambient temperatures using a Varian $400 \mathrm{MHz}$ or 500 $\mathrm{MHz}$ spectrometer. Chemical shifts are reported in parts per million (ppm) relative to the internal solvent peak (Chloroform- $d: \delta 7.26$ for ${ }^{1} \mathrm{H} ; \delta 77.16$ for ${ }^{13} \mathrm{C}$. Methanol- $d_{4}: \delta 3.31$ for ${ }^{1} \mathrm{H} ; \delta 49.00$ for ${ }^{13} \mathrm{C}$ ). Data for ${ }^{1} \mathrm{H}$ NMR are reported as follows: chemical shift, multiplicity $(b r=$ broad, $\mathrm{s}=$ singlet, $\mathrm{d}=$ doublet, $\mathrm{t}=$ triplet, $\mathrm{q}=$ quartet, $\mathrm{m}=$ multiplet ), coupling constants, and integration. All ${ }^{13} \mathrm{C}$ NMR spectra were recorded with complete proton decoupling. Infrared spectra were recorded on a Bruker ALPHA P FT-IR spectrometer equipped with a diamond ATR module. Highresolution mass spectra were obtained at the Boston University Chemical Instrumentation Center using a Waters Q-TOF mass spectrometer. Melting points were recorded on a Mel-temp apparatus (Laboratory Devices) and are uncorrected. Analytical LCMS was performed on a Waters Acquity UPLC (Ultra Performance Liquid Chromatography (Waters MassLynx Version 4.1) with a Binary solvent manager, SQ mass spectrometer, Waters 2996 PDA (PhotoDiode Array) detector, and ELSD (Evaporative Light Scattering Detector). An Acquity UPLC BEH C18 $1.7 \mu \mathrm{m}$ column was used for analytical UPLC-MS. Analytical thin layer chromatography (TLC) was performed using $0.25 \mathrm{~mm}$ silica gel 60-F plates (Merck KGaA). Preparative HPLC was performed on a Gilson PLC2020 using a Waters SunFire ${ }^{\text {TM }}$ Prep C18 OBD ${ }^{\text {TM }} 5 \mu \mathrm{m} 19 \times 50$ mm column. Flash chromatography was performed using ZEOprep 60 Eco 40-63 $\mu \mathrm{m}$ silica gel (Zeochem AG). Preparative TLC was conducted with glass-backed $250 \mu \mathrm{m}$ silica gel 60-F plates (Merck KGaA). Yields refer to chromatographically and spectroscopically pure compounds, unless otherwise stated. Optical rotations were recorded on an AUTOPUL III digital polarimeter at $589 \mathrm{~nm}$ and are recorded as $[\alpha]_{\mathrm{D}}{ }^{\text {temp. }}$ (concentration in grams $/ 100 \mathrm{~mL}$ solvent). Chiral HPLC analysis of enantioenriched compounds was performed using a Waters 1525 Binary HPLC Pump with a Waters 2487 diode array detector. A CHIRAL ${ }^{\circledR}$ OD-H [Chiral Technologies Inc., $150 \times 4.60 \mathrm{~mm}$ 
$(\mathrm{L} \times$ I.D.) ] column was used for enantiomeric excess determination. All reactions were carried out in oven-dried glassware under an argon atmosphere unless otherwise noted. The Scilligence ELN Reaction Planner (Scilligence Corp.) was used for experimental procedure planning.

\section{B. Reagents and Solvents}

HPLC grade methylene chloride and acetonitrile were purchased from Fisher and methylene chloride was purified and dried by passing through a PURE SOLV ${ }^{\circledR}$ solvent purification system (Innovative Technology, Inc.). Anhydrous acetonitrile for photoreactions was purchased from Sigma Aldrich and was used as received. Spectrophotometric grade solvents for photophysical experiments were purchased from Sigma Aldrich and was used as received, including acetonitrile, methylcyclohexane, and ethanol. All other reagents were purchased and used as received from Alfa Aesar, Oakwood Chemical, Sigma Aldrich, Strem, and TCI America.

\section{Photoreaction Setup}

\section{Rayonet Recirculating Flow Reactor:}

The flow system is described in detail in our literature reference. ${ }^{\mathrm{S} 1}$

\section{Plain Flow Photoreactor I:}

An LED flow reactor was constructed using PTFE tubing (Cole-Parmer Instrument Company, 1/32"ID x 1/16"OD) twined into a plate shape on an aluminum support (Figure S1A). Available tubing volume was measured to be $2.7 \mathrm{~mL}$. The reactor was placed in a flammable cabinet equipped with a purple LED lamp $\left(\operatorname{Kessil}^{\circledR}, 40 \mathrm{~W}, \lambda_{\mathrm{em}}=370-420 \mathrm{~nm}, \lambda_{\text {peak }}=390 \mathrm{~nm}\right)$ and a fan (Figure S1B). The inlet of tubing was connected to a syringe pump (KD Scientific, LEGATO 100 Syringe Pump). Connecting parts were all purchased from IDEX Health \& Science. 


\section{Temperature-Controlled Flow Photoreactor II:}

A temperature-controlled flow reactor was constructed using PTFE tubing twined onto a beaker (Figures S2A and B). The beaker was placed in a slightly larger plastic container and the top was sealed with hot glue. The available tubing volume was measured to be $6.0 \mathrm{~mL}$. The interlayer is connected to a circular chiller with glycol controlling the reactor temperature. A small convex mirror was placed at the bottom of the reactor in order to improve light focusing efficiency. The reactor was placed in a flammable cabinet equipped with a purple LED lamp (Figure S2C) on top. The tubing inlet was finally connected to a syringe pump.

\section{Batch Reactor III:}

A batch photochemical reactor was built in a flammable cabinet with a stirring plate and a purple LED lamp (Figure S3). A fan was placed above the lamp to prevent overheating. Meanwhile, a large piece of aluminum foil was placed behind the test tubes to enhance light focusing. Reaction Pyrex ${ }^{\circledR}$ tubes were placed on the stirring plate with a tube rack; the distance between the tubes and lamp was kept within $5 \mathrm{~cm}$.

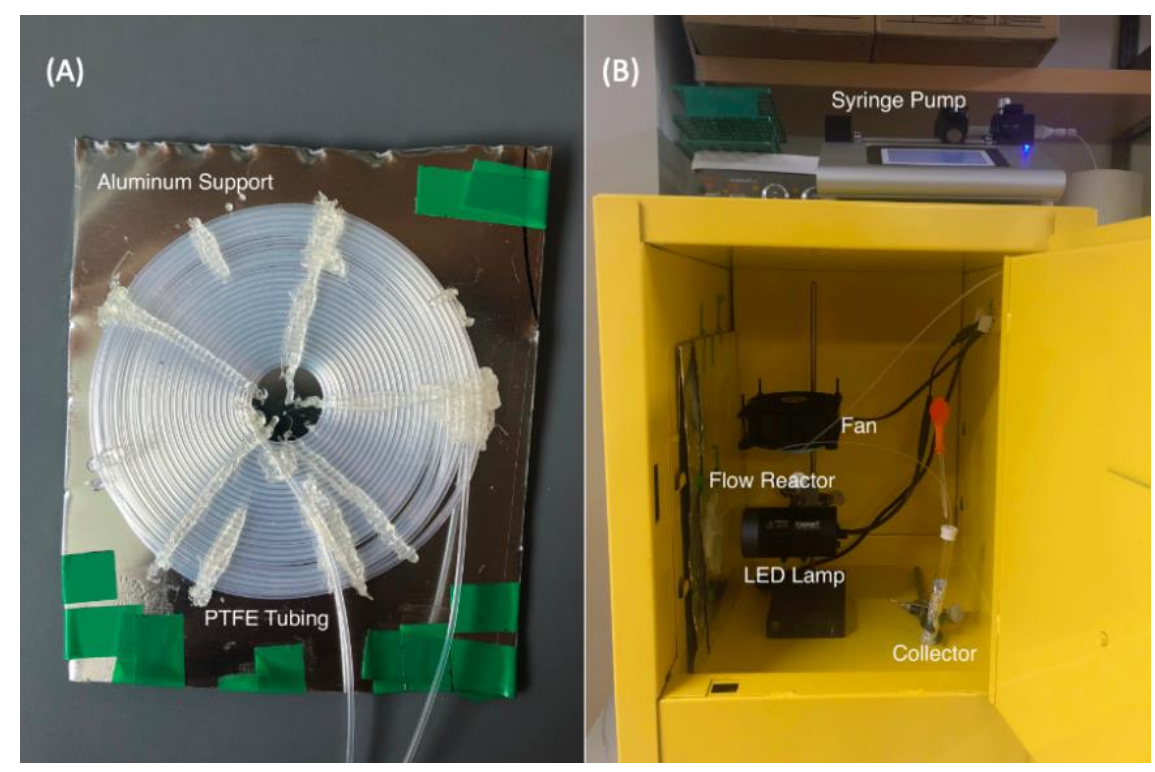

Figure S1. Plain flow photoreactor I. 

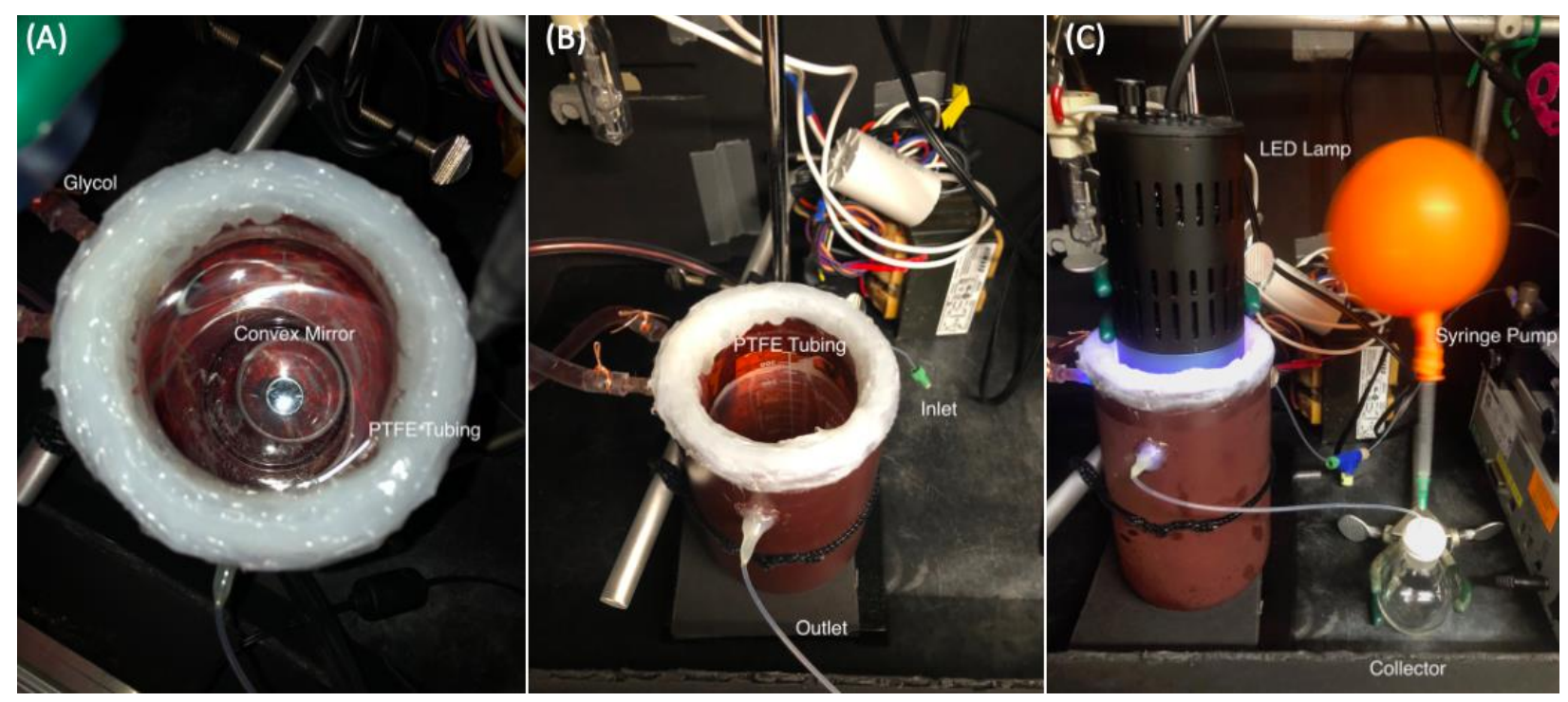

Figure S2. Temperature-controlled flow photoreactor II.

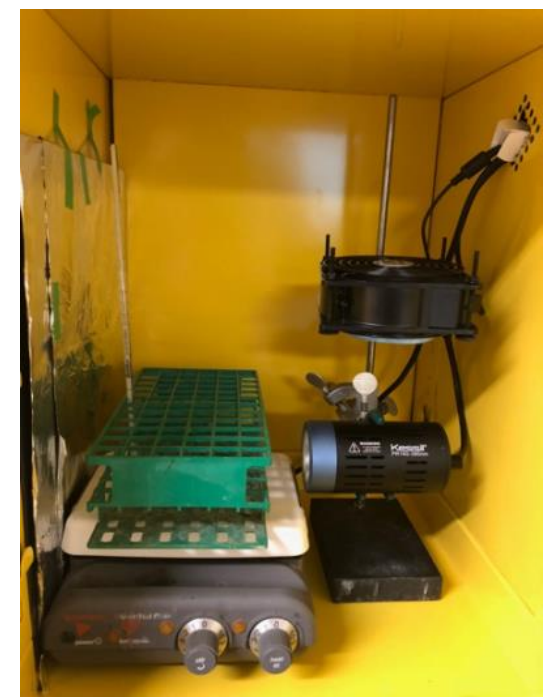

Figure S3. Batch reactor III. 


\section{Experimental Procedures and Compound Characterization}

\section{A. Dearomatization}

Experimental Procedure: The procedure was followed and modified according to literature references. ${ }^{\mathrm{S} 2-4}$

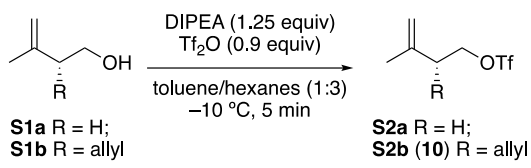

General Procedure for the Synthesis of Alkyl Triflates: ${ }^{\mathrm{S} 2,3}$ To a flame-dried flask containing alcohol S1 (1.0 equiv) was added toluene and hexane (1:3, $0.4 \mathrm{M})$ and diisopropylethylamine (DIPEA, 1.25 equiv) under argon. The solution was cooled to $-10{ }^{\circ} \mathrm{C}$, and trifluoromethanesulfonic anhydride ( $\mathrm{Tf}_{2} \mathrm{O}, 0.9$ equiv) was then added in one portion. The reaction mixture was allowed to stir for $5 \mathrm{~min}$ before it was quickly filtered through a cotton plug. The clear solution of primary alkyl triflate $\mathbf{S 2}$ was then used immediately without further purification.
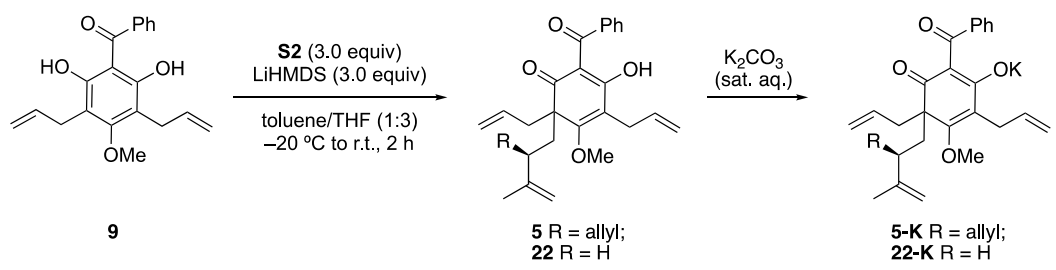

General Dearomatization Procedure A: ${ }^{\mathrm{S} 2}$ To a flame-dried 50-mL round-bottom flask with benzoylated phloroglucinol derivative $9^{\mathrm{S} 2}(162 \mathrm{mg}, 0.5 \mathrm{mmol})$ was added a mixture of toluene and THF $(8 \mathrm{~mL}, 1: 3,62.5 \mathrm{mM})$ under argon, and the mixture was then cooled to $-20^{\circ} \mathrm{C}$. A solution of LiHMDS (1.0 M in THF, $1.47 \mathrm{~mL}, 3.0$ equiv) was added over the course of 2 min leading to a homogeneous dark-red solution. After the mixture was stirred for $5 \mathrm{~min}$, the solution of triflate $\mathbf{S 2}$ (3.0 equiv) was added dropwise at a steady rate. The reaction was allowed to warm to r.t. and was stirred for $2 \mathrm{~h}$. After completion, the reaction was quenched with $1 \mathrm{~N} \mathrm{HCl}$ solution, poured into water, and extracted with $\mathrm{CH}_{2} \mathrm{Cl}_{2}(50 \mathrm{~mL} \times 3)$. The combined organic layers were washed with water and brine, dried over anhydrous $\mathrm{Na}_{2} \mathrm{SO}_{4}$, filtered, and concentrated in vacuo. The dearomatized compound was then dissolved in $n$-pentane and was washed with saturated $\mathrm{K}_{2} \mathrm{CO}_{3}$ 
aqueous solution to afford its potassium salt as a yellow precipitate. This potassium salt was used for characterization purposes.

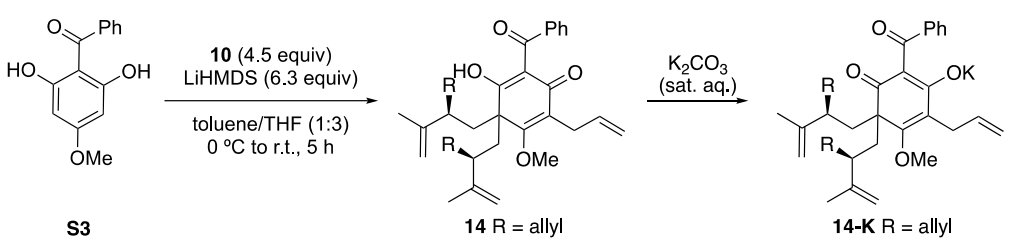

General Dearomatization Procedure B: ${ }^{\mathrm{S} 3}$ To a flame-dried, argon-charged $50-\mathrm{mL}$ round-bottom flask with phloroglucinol derivative $\mathbf{S 3}^{\mathrm{S} 2}$ (244 $\mathrm{mg}, 1.0 \mathrm{mmol}$ ) was added a mixture of toluene and THF (16 mL, 1:3, $62.5 \mathrm{mM})$ under argon, and the mixture was then cooled to $0{ }^{\circ} \mathrm{C}$. A solution of LiHMDS (1.0 M in THF, $6.3 \mathrm{~mL}, 6.3$ equiv) was added over the course of $5 \mathrm{~min}$. To the mixture was then added triflate $\mathbf{1 0}$ (4.5 equiv) in two portions at a steady rate. The mixture was allowed to warm to room temperature after the completion of addition and stirred for $5 \mathrm{~h}$. The reaction was quenched with $1 \mathrm{~N} \mathrm{HCl}$ solution $(50 \mathrm{~mL})$, diluted with water $(100 \mathrm{~mL})$, and was extracted with diethyl ether $(50 \mathrm{~mL} \times 3)$. The combined organic layers were washed with $1 \mathrm{~N} \mathrm{HCl}$ solution, water, and brine. The organic solution was then dried over anhydrous $\mathrm{Na}_{2} \mathrm{SO}_{4}$, filtered, and was concentrated in vacuo. Purification by silica flash chromatography (EtOAc/hexanes) provided the desired dearomatized compound. The dearomatized compound was then dissolved in $n$-pentane and washed with saturated $\mathrm{K}_{2} \mathrm{CO}_{3}$ aqueous solution to afford its potassium salt as a yellow precipitate which was used for characterization purposes.

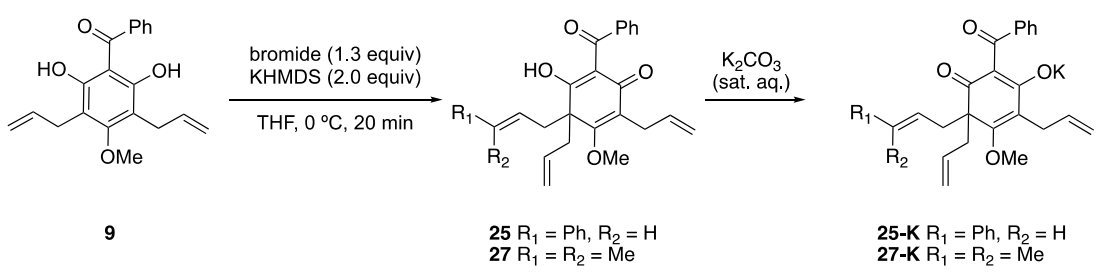

General Dearomatization Procedure C: ${ }^{\mathrm{S} 4}$ To a solution of 9 (150 mg, $0.46 \mathrm{mmol}, 1.0$ equiv) in THF ( $5 \mathrm{~mL})$ was added KHMDS (1 M, $0.92 \mathrm{~mL}, 2.0$ equiv) at $0{ }^{\circ} \mathrm{C}$ under argon. After 2 $\mathrm{min}$, an allylic bromide (1.3 equiv) was added dropwise as a solution in benzene (450 mg/mL). After $20 \mathrm{~min}$, the reaction was poured into saturated aqueous $\mathrm{NH}_{4} \mathrm{Cl}(10 \mathrm{~mL})$ and the mixture was extracted three times with diethyl ether $(10 \mathrm{~mL} \times 3)$. The combined organic layers were washed with brine, dried over anhydrous $\mathrm{Na}_{2} \mathrm{SO}_{4}$, and concentrated in vacuo. The crude residue was purified by silica gel chromatography (EtOAc/hexanes: 5/95 to 25/75) to afford desired product. The dearomatized product was then dissolved in $n$-pentane and washed with saturated $\mathrm{K}_{2} \mathrm{CO}_{3}$ 
aqueous solution to afford its potassium salt as a yellow precipitate. This potassium salt was used for characterization purposes.

Dearomatized Substrate 5: ${ }^{\mathrm{S} 2} 180 \mathrm{mg}, 83 \%$ yield; slightly yellow oil; $\mathrm{R}_{f}=0.32$ (EtOAc/hexanes: 10/90); ${ }^{1} \mathrm{H}$ NMR (potassium enolate form; 1.3:1 mixture of diastereomers as determined by ${ }^{1} H$ NMR analysis, $\left.\mathrm{CD}_{3} \mathrm{OD}, 400 \mathrm{MHz}\right): \delta 7.77-7.73(\mathrm{~m}, 1.8 \mathrm{H}), 7.40-7.37(\mathrm{~m}, 1.0 \mathrm{H})$, 7.31-7.28 (m, 1.9H), 6.00-5.93 (m, 1.1H), 5.78-5.69 (m, 1.1H), 5.65-5.56 (m, 1.1H), 5.10-5.01 $(\mathrm{m}, 2.1 \mathrm{H}), 4.95-4.93(\mathrm{~m}, 2.2 \mathrm{H}), 4.89-4.80(\mathrm{~m}, 2.6 \mathrm{H}), 4.60-4.56(\mathrm{~m}, 1.7 \mathrm{H}), 3.96(\mathrm{~s}, 1.0 \mathrm{H}), 3.91$ (s, 1.3H), 3.22-3.12 (m, 2.0H), 2.62-2.56 (m, 1.2H), 2.50-2.42 (m, 1.2H), 2.24-2.18 (m, 1.8H), 2.07-1.92 (m, 2.8H), 1.84-1.65 (m, 1.2H), 1.60-1.57 (m, 2.5H), 1.29-1.25 (m, 1.2H).

Dearomatized Substrate 14: ${ }^{\mathrm{S} 3} 217 \mathrm{mg}, 46 \%$ yield; beige amorphous solid; $\mathrm{R}_{f}=0.20$ (EtOAc/hexanes: 10/90); ${ }^{1} \mathrm{H}$ NMR (potassium enolate form, $\left.\mathrm{CD}_{3} \mathrm{OD}, 500 \mathrm{MHz}\right): \delta 7.77$ (d, J=7.5 $\mathrm{Hz}, 2 \mathrm{H}), 7.40(\mathrm{t}, J=7.5 \mathrm{~Hz}, 1 \mathrm{H}), 7.31(\mathrm{~d}, J=7.5 \mathrm{~Hz}, 2 \mathrm{H}), 5.64-5.56(\mathrm{~m}, 2 \mathrm{H}), 5.21(\mathrm{~s}, 1 \mathrm{H}), 4.91-$ $4.82(\mathrm{~m}, 4 \mathrm{H}), 4.61(\mathrm{~d}, J=3.6 \mathrm{~Hz}, 2 \mathrm{H}), 3.52$ (s, 3H), 2.24-2.19 (m, 2H), 2.15-2.10 (m, 2H), 2.00$1.94(\mathrm{~m}, 2 \mathrm{H}), 1.88(\mathrm{dd}, J=13.5,7.5 \mathrm{~Hz}, 2 \mathrm{H}), 1.82(\mathrm{dd}, J=13.0,2.0 \mathrm{~Hz}, 2 \mathrm{H}), 1.59(\mathrm{~s}, 6 \mathrm{H})$.

Dearomatized Substrate 22: $133 \mathrm{mg}$, 69\% yield; slightly yellow oil; $\mathrm{R}_{f}=0.45$ (EtOAc/hexanes: 10/90); ${ }^{1} \mathrm{H}$ NMR (potassium enolate form, $\left.\mathrm{CD}_{3} \mathrm{OD}, 400 \mathrm{MHz}\right): \delta 7.79(\mathrm{~d}, J=7.2$ $\mathrm{Hz}, 2 \mathrm{H}), 7.41(\mathrm{t}, J=7.2 \mathrm{~Hz}, 1 \mathrm{H}), 7.32$ (t, $J=7.2 \mathrm{~Hz}, 2 \mathrm{H}), 6.01-5.91(\mathrm{~m}, 1 \mathrm{H}), 5.79-5.69(\mathrm{~m}, 1 \mathrm{H})$, $5.05(\mathrm{td}, J=15.2,2.0 \mathrm{~Hz}, 2 \mathrm{H}), 4.96(\mathrm{td}, J=9.6,1.6 \mathrm{~Hz}, 2 \mathrm{H}), 4.63(\mathrm{~s}, 2 \mathrm{H}), 3.91(\mathrm{~s}, 3 \mathrm{H}), 3.20(\mathrm{~d}, J$ $=5.6 \mathrm{~Hz}, 2 \mathrm{H}), 2.64-2.58(\mathrm{~m}, 1 \mathrm{H}), 2.53-2.48(\mathrm{~m}, 1 \mathrm{H}), 2.04-1.95(\mathrm{~m}, 2 \mathrm{H}), 1.88-1.81(\mathrm{~m}, 2 \mathrm{H}), 1.68$ (s, 3H); ${ }^{13} \mathrm{C}$ NMR $\left(\mathrm{CD}_{3} \mathrm{OD}, 101 \mathrm{MHz}\right): \delta 201.1,192.0,187.5,169.6,147.2,142.5,139.0,135.9$, 132.2, 130.1, 128.7, 123.3, 117.3, 116.9, 114.7, 109.9, 62.7, 57.6, 43.9, 37.5, 34.0, 29.4, 22.8; IR $_{v \max }$ (Diamond ATR): 2930, 1634, 1600, 1516, 1429, 1343, 1225, 1133, 993, 917, 696, $634 \mathrm{~cm}^{-}$ 1; HRMS (ESI): [M + H] $]^{+}$Calcd. For $\mathrm{C}_{25} \mathrm{H}_{29} \mathrm{O}_{4}$ : 393.2066, Found: 393.2055.

Dearomatized Substrate 25: ${ }^{\mathrm{S} 4} 144 \mathrm{mg}, 71 \%$ yield; colorless oil; $\mathrm{R}_{f}=0.44$ (EtOAc/hexanes: 25/75); ${ }^{1} \mathrm{H}$ NMR (potassium enolate form, $\left.\mathrm{CD}_{3} \mathrm{OD}, 500 \mathrm{MHz}\right): \delta 7.70(\mathrm{t}, J=7.5$ $\mathrm{Hz}, 2 \mathrm{H}), 7.31-7.24(\mathrm{~m}, 5 \mathrm{H}), 7.18(\mathrm{t}, J=7.5 \mathrm{~Hz}, 1 \mathrm{H}), 7.07(\mathrm{t}, J=7.5 \mathrm{~Hz}, 2 \mathrm{H}), 6.42(\mathrm{~d}, J=16.0$ $\mathrm{Hz}, 1 \mathrm{H}), 6.17(\mathrm{dt}, J=16.0,7.0 \mathrm{~Hz}, 1 \mathrm{H}), 5.94-5.86(\mathrm{~m}, 1 \mathrm{H}), 5.77-5.69(\mathrm{~m}, 1 \mathrm{H}), 5.04(\mathrm{td}, J=14.0$, 
$1.5 \mathrm{~Hz}, 2 \mathrm{H}), 4.95(\mathrm{~d}, J=10.5 \mathrm{~Hz}, 1 \mathrm{H}), 4.86(\mathrm{dd}, J=9.5,2.0 \mathrm{~Hz}, 1 \mathrm{H}), 3.97(\mathrm{~s}, 3 \mathrm{H}), 3.19$ (ddd, $J=$ $30.5,16,5.5 \mathrm{~Hz}, 2 \mathrm{H}), 2.77-2.66(\mathrm{~m}, 3 \mathrm{H}), 2.55(\mathrm{dd}, J=13.0,8.0 \mathrm{~Hz}, 1 \mathrm{H}) ;{ }^{13} \mathrm{C} \mathrm{NMR}\left(\mathrm{CD}_{3} \mathrm{OD}, 126\right.$ MHz) $\delta 201.4,191.4,187.4,169.5,142.3,139.1,138.9,135.9,133.4,132.1,130.2,129.4,128.7$, $128.0,127.3,127.2,122.8,117.2,117.0,114.7,62.7,58.1,43.3,42.5,29.2$.

Dearomatized Substrate 27: $87 \mathrm{mg}$, 48\% yield; colorless oil; $\mathrm{R}_{f}=0.54$ (EtOAc/hexanes: 25/75); ${ }^{1} \mathrm{H}$ NMR (potassium enolate form, $\mathrm{CD}_{3} \mathrm{OD}, 500 \mathrm{MHz}$ ): $\delta 7.43$ (d, $J=7.5 \mathrm{~Hz}, 2 \mathrm{H}$ ), 7.39 (t, $J=7.0 \mathrm{~Hz}, 1 \mathrm{H}), 7.30(\mathrm{t}, J=7.0 \mathrm{~Hz}, 2 \mathrm{H}), 5.86-5.89(\mathrm{~m}, 1 \mathrm{H}), 5.74-5.65(\mathrm{~m}, 1 \mathrm{H}), 5.09-5.00(\mathrm{~m}$, $3 \mathrm{H}), 4.92(\mathrm{~d}, J=10 \mathrm{~Hz}, 2 \mathrm{H}), 3.23-3.14(\mathrm{~m}, 2 \mathrm{H}), 2.64(\mathrm{dd}, J=12.5,5.5 \mathrm{~Hz}, 1 \mathrm{H}), 2.56-2.45(\mathrm{~m}$, $3 \mathrm{H}), 1.66$ (s, 3H), 1.59 (s, 3H); ${ }^{13} \mathrm{C}$ NMR (CD $\left.{ }_{3} \mathrm{OD}, 126 \mathrm{MHz}\right): \delta 201.0,192.2,187.7,169.9,142.6$, 139.0, 136.1, 133.7, 132.0, 130.2, 128.6, 122.8, 121.7, 117.1, 116.8, 114.5, 62.5, 57.6, 43.3, 37.9, 29.3, 26.1, 18.3; IR $v_{\max }$ (Diamond ATR): 2917, 1640, 1516, 1428, 1228, 1134, 993, 918, $696 \mathrm{~cm}^{-}$ ${ }^{1}$; HRMS (ESI): [M + H] ${ }^{+}$Calcd. For $\mathrm{C}_{25} \mathrm{H}_{29} \mathrm{O}_{4}$ : 393.2066, Found: 393.2051.

\section{B. Photocyclization of Dearomatized Substrate and Condition Optimization}

General Experimental Procedure A using Flow Photoreactor I: Dearomatized substrate (20 mg) and additive were dissolved in anhydrous solvent and the mixture was degassed using sonication for $30 \mathrm{~min}$ before being taken up into a syringe. The reaction mixture was then injected in the flow photoreactor I with a syringe pump at a constant rate which was followed by a flush of the corresponding solvent at the same rate until all of the reaction solution was eluted from the flow reactor and collected. The solvent was then removed in vacuo and the resulting residue was purified by silica flash chromatography or preparative thin-layer chromatography (EtOAc/hexanes). Unless otherwise noted, all the photoreactions were conducted using these general conditions.

General Experimental Procedure B using Flow Reactor II: Dearomatized substrate (20 $\mathrm{mg}$ ) was dissolved in anhydrous acetonitrile $(5 \mathrm{mM})$ and the mixture was degassed using sonication for $30 \mathrm{~min}$ before taken into a syringe. The reaction mixture was then injected in the flow photoreactor II with a syringe pump at a constant rate $\left(16.67 \mu \mathrm{L} / \mathrm{min}, t_{\mathrm{R}}=6 \mathrm{~h}\right)$ which was 
followed by a flush with acetonitrile at the same rate until all of the reaction solution was eluted from the flow reactor and collected. The chiller was able to keep the reaction temperature at $10{ }^{\circ} \mathrm{C}$. The solvent was then removed in vacuo and the resulting residue was purified by silica flash chromatography or preparative thin-layer chromatography (EtOAc/hexanes).

\section{Table S1. Condition screening for the photocyclization of 5.}

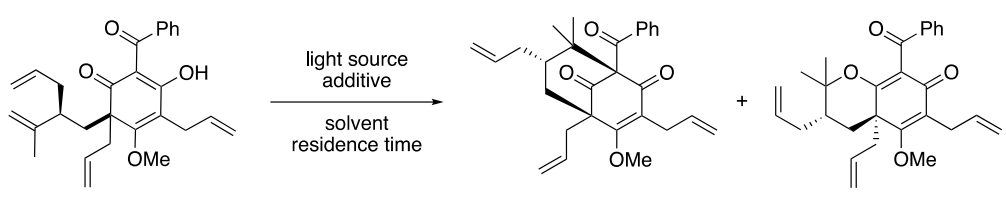

5

$\begin{array}{lll}(-)-8 & 11\end{array}$

\begin{tabular}{|c|c|c|c|c|c|}
\hline Entry & Light Source $\lambda_{\max }(\mathrm{nm})$ & Solvent (mM) & Additive (mol\%) & Residence Time $t_{\mathrm{R}}(\mathrm{h})$ & Yield $^{\mathrm{a}}(\%)$ \\
\hline $1^{\mathrm{b}}$ & 350 & $\mathrm{CH}_{3} \mathrm{CN}(0.5)$ & $\mathrm{n} / \mathrm{a}$ & 12 & $(-)-8: 16,11: 12$ \\
\hline 2 & 390 & $\mathrm{CH}_{3} \mathrm{CN}(5)$ & $\mathrm{n} / \mathrm{a}$ & 2 & $(-)-8: 22.5,11: 7$ \\
\hline 3 & 427 & $\mathrm{CH}_{3} \mathrm{CN}(5)$ & $\mathrm{n} / \mathrm{a}$ & 2 & n.r. ${ }^{\mathrm{c}}$ \\
\hline 4 & metal halide & $\mathrm{CH}_{3} \mathrm{CN}(5)$ & $\mathrm{n} / \mathrm{a}$ & 2 & $(-)-8: 9$ \\
\hline 5 & 390 & toluene (5) & $\mathrm{n} / \mathrm{a}$ & 2 & n.r. \\
\hline 6 & 390 & $\mathrm{CH}_{2} \mathrm{Cl}_{2}(5)$ & $\mathrm{n} / \mathrm{a}$ & 2 & $(-)-8: 5$ \\
\hline 7 & 390 & $\mathrm{CH}_{2} \mathrm{Cl}_{2} / \mathrm{TFE}$ (5) & $\mathrm{n} / \mathrm{a}$ & 2 & trace \\
\hline 8 & 390 & THF (5) & $\mathrm{n} / \mathrm{a}$ & 2 & n.r. \\
\hline 9 & 390 & $\mathrm{CH}_{3} \mathrm{CN}(5)$ & $\mathrm{n} / \mathrm{a}$ & 1 & trace \\
\hline 10 & 390 & $\mathrm{CH}_{3} \mathrm{CN}(5)$ & $\mathrm{n} / \mathrm{a}$ & 4 & $(-)-8: 24,11: 6$ \\
\hline 11 & 390 & $\mathrm{CH}_{3} \mathrm{CN}(5)$ & $\mathrm{n} / \mathrm{a}$ & 8 & decomp. \\
\hline $12^{\mathrm{d}}$ & 370 & $\mathrm{CH}_{3} \mathrm{CN}(5)$ & thioxanthone ${ }^{\mathrm{e}}(5)$ & 0.5 & decomp. \\
\hline $13^{\mathrm{d}}$ & 390 & $\mathrm{CH}_{3} \mathrm{CN}(5)$ & dibromothioxanthone $^{\mathrm{e}}(5)$ & 0.5 & decomp. \\
\hline 14 & 390 & $\mathrm{CH}_{3} \mathrm{CN}(1)$ & $\mathbf{n} / \mathbf{a}$ & 4 & $(-)-8: 29,11: 10$ \\
\hline 15 & 390 & $\mathrm{CH}_{3} \mathrm{CN}(10)$ & $\mathrm{n} / \mathrm{a}$ & 4 & $(-)-\mathbf{8}: 19, \mathbf{1 1}: 9$ \\
\hline $16^{\mathrm{f}}$ & $\mathrm{n} / \mathrm{a}$ & 1,4-dioxane & $\mathrm{n} / \mathrm{a}$ & 36 & n.r. \\
\hline
\end{tabular}

${ }^{a}$ Isolated yield; ${ }^{b}$ Reaction was conducted in Rayonet flow system ( see S1) around $-20{ }^{\circ} \mathrm{C}$ for 12 h; ${ }^{c}$ n.r.: No reaction; ${ }^{d}$ Reaction was conducted as a batch process for $0.5 \mathrm{~h}$ (see general procedure C); ${ }^{e}$ For more details about thioxanthone and its derivatives, see reference S5; ${ }^{f}$ Thermal control conditions.

General Experimental Procedure C in Batch: To a flame-dried test tube was added dearomatized substrate $(20 \mathrm{mg})$ and anhydrous acetonitrile $(5 \mathrm{mM})$ and the mixture was degassed using sonication for $30 \mathrm{~min}$. After degassing, the test tube was placed in front of a Kessil ${ }^{\circledR}$ LED lamp and was allowed to stir for $36 \mathrm{~h}$ at room temperature. After the completion, the solvent was removed in vacuo and the residue was purified by silica flash chromatography or preparative thinlayer chromatography (EtOAc/hexanes). 
Control Experiment under Thermal Conditions (Conia-Ene Conditions): To an ovendried microwave reaction vial was added dearomatized substrate 5 (20 mg) and anhydrous 1,4dioxane $(5 \mathrm{mM})$ and the mixture was degassed using sonication for $10 \mathrm{~min}$. After degassing, the vial was sealed with a polyethylene snap cap was placed in microwave (CEM Discover SP, 300 W) and was heated to $100{ }^{\circ} \mathrm{C} / 300 \mathrm{psi}$ for $1 \mathrm{~h}$. After cooling of the microwave reactor, the solvent was removed in vacuo and the residue was purified by preparative thin-layer chromatography (EtOAc/hexanes).

PPAP Core (-)-8: colorless oil; $\mathrm{R}_{f}=0.30$ (EtOAc/hexanes: 10/90); ${ }^{1} \mathrm{H}$ NMR $\left(\mathrm{CDCl}_{3}, 500\right.$ MHz): $\delta 7.43(\mathrm{dd}, J=8.5,1.0 \mathrm{~Hz}, 2 \mathrm{H}), 7.38(\mathrm{tt}, J=7.5,1.0 \mathrm{~Hz}, 1 \mathrm{H}), 7.24(\mathrm{td}, J=7.5,0.5 \mathrm{~Hz}, 2 \mathrm{H})$, 5.86-5.74 (m, 2H), 5.69-5.61 (m, 1H), $5.17(\mathrm{dd}, J=17.5,2.0 \mathrm{~Hz}, 1 \mathrm{H}), 5.11-5.01(\mathrm{~m}, 5 \mathrm{H}), 4.08$ (s, 3H), 3.37 (ddt, $J=16.0,6.5,1.5 \mathrm{~Hz}, 1 \mathrm{H}), 3.24$ (ddt, $J=16.0,6.0,1.5 \mathrm{~Hz}, 1 \mathrm{H}), 2.71$ (dd, $J=$ 14.0, $6.0 \mathrm{~Hz}, 1 \mathrm{H}), 2.51(\mathrm{dd}, J=13.5,7.5 \mathrm{~Hz}, 1 \mathrm{H}), 2.38-2.34(\mathrm{~m}, 1 \mathrm{H}), 2.06(\mathrm{dd}, J=14.0,4.0 \mathrm{~Hz}$, 1H), 1.77-1.07 (m, 2H), 1.54-1.47 (m, 1H), $1.34(\mathrm{~s}, 3 \mathrm{H}), 1.14(\mathrm{~s}, 3 \mathrm{H}) ;{ }^{13} \mathrm{C} \mathrm{NMR}\left(\mathrm{CDCl}_{3}, 126\right.$ MHz): $\delta$ 206.7, 193.7, 192.9, 173.0, 136.7, 136.2, 135.4, 133.5, 132.0, 128.4, 127.9, 123.4, 118.8, 117.0, 115.8, 79.1, 62.4 59.4, 48.7, 42.3, 40.2, 35.1, 32.8, 28.2, 23.3, 15.8; IRvmax (Diamond ATR): 2942, 1724, 1699, 1642, 1597, 1447, 1341, 1240, 917, $735 \mathrm{~cm}^{-1}$; HRMS (ESI): $[\mathrm{M}+\mathrm{H}]^{+}$Calcd. For $\mathrm{C}_{28} \mathrm{H}_{33} \mathrm{O}_{4}: 433.2379$, Found: 433.2384; $[\alpha]_{\mathrm{D}}^{23}=-74.9\left(\mathrm{c}=0.200, \mathrm{CHCl}_{3}\right)$.

$\boldsymbol{O}$-Cyclized Byproduct 11: ${ }^{\mathrm{S} 2}$ colorless oil; $\mathrm{R}_{f}=0.15$ (EtOAc/hexanes: 10/90); ${ }^{1} \mathrm{H}$ NMR $\left(\mathrm{CDCl}_{3}, 400 \mathrm{MHz}\right): \delta 7.87(\mathrm{dd}, J=8.0,1.6 \mathrm{~Hz}, 2 \mathrm{H}), 7.51(\mathrm{tt}, J=7.2,2.0 \mathrm{~Hz}, 1 \mathrm{H}), 7.40(\mathrm{t}, J=8.0$ $\mathrm{Hz}, 2 \mathrm{H}), 5.95-5.85(\mathrm{~m}, 1 \mathrm{H}), 5.75-5.58(\mathrm{~m}, 2 \mathrm{H}), 5.17-4.98(\mathrm{~m}, 6 \mathrm{H}), 3.98(\mathrm{~s}, 3 \mathrm{H}), 3.22(\mathrm{~d}, J=6.4$ $\mathrm{Hz}, 2 \mathrm{H}), 2.76-2.66(\mathrm{~m}, 2 \mathrm{H}), 2.47(\mathrm{dd}, J=14.4,4.0 \mathrm{~Hz}, 1 \mathrm{H}), 2.22-2.16(\mathrm{~m}, 1 \mathrm{H}), 1.81-1.73(\mathrm{~m}$, $1 \mathrm{H}), 1.67-1.59(\mathrm{~m}, 1 \mathrm{H}), 1.55-1.50(\mathrm{~m}, 1 \mathrm{H}), 1.28(\mathrm{~s}, 3 \mathrm{H}), 1.19(\mathrm{~s}, 3 \mathrm{H}) ;{ }^{13} \mathrm{C} \mathrm{NMR}\left(\mathrm{CDCl}_{3}, 101 \mathrm{MHz}\right)$ $\delta 194.6,186.4,171.4,169.9,137.8,136.5,136.0,133.2$, 132.4, 129.5, 128.4, 126.0, 121.0, 119.8, 117.4, $115.2,88.3,62.3,49.8,46.6,42.7,35.6,33.9,28.7,28.4,21.8$.

PPAP Core (-)-15: 10.9 mg, 54\% yield; white solid, m. p.: 133-135 C (EtOAc/hexanes: 20/80); $\mathrm{R}_{f}=0.50$ (EtOAc/hexanes: $\left.25 / 75\right) ;{ }^{1} \mathrm{H} \mathrm{NMR}\left(\mathrm{CDCl}_{3}, 500 \mathrm{MHz}\right): \delta 7.63$ (dd, $J=7.5,1.0$ $\mathrm{Hz}, 2 \mathrm{H}), 7.43(\mathrm{tt}, J=7.0,1.0 \mathrm{~Hz}, 1 \mathrm{H}), 7.31(\mathrm{t}, J=7.5 \mathrm{~Hz}, 2 \mathrm{H}), 5.88(\mathrm{~s}, 1 \mathrm{H}), 5.65-5.56(\mathrm{~m}, 2 \mathrm{H})$, $5.03-4.90(\mathrm{~m}, 4 \mathrm{H}), 4.64(\mathrm{dd}, J=23.0,2.0 \mathrm{~Hz}, 2 \mathrm{H}), 3.74(\mathrm{~s}, 3 \mathrm{H}), 2.39-2.32(\mathrm{~m}, 2 \mathrm{H}), 2.17-1.98(\mathrm{~m}$, 
4H), 1.84-1.78 (m, 2H), 1.74-1.67 (m, 1H), 1.65 (s, 3H), 1.44-1.36 (m, 1H), 1.39 (s, 3H), 1.11 (s, $3 \mathrm{H}) ;{ }^{13} \mathrm{C} \mathrm{NMR}\left(\mathrm{CDCl}_{3}, 126 \mathrm{MHz}\right): \delta 207.5,193.1,177.0,148.4,136.97,136.96,136.4,132.4$, 128.6, 128.1, 117.0, 115.7, 110.8, 105.6, 79.7, 57.7, 55.8, 48.9, 44.7, 42.2, 41.7, 39.5, 33.6, 33.0,

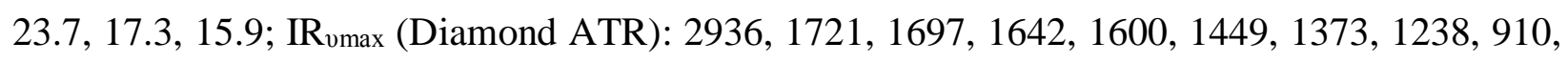
$735 \mathrm{~cm}^{-1}$; HRMS (ESI): $[\mathrm{M}+\mathrm{H}]^{+}$Calcd. For $\mathrm{C}_{30} \mathrm{H}_{37} \mathrm{O}_{4}: 461.2692$, Found: 461.2693; $[\alpha]_{\mathrm{D}}^{24}=-59.3$ $\left(\mathrm{c}=0.100, \mathrm{CHCl}_{3}\right)$.

$\boldsymbol{O}$-Cyclized Byproduct 16: ${ }^{\mathrm{S} 3} 2.2 \mathrm{mg}, 11 \%$ yield; white amorphous solid; $\mathrm{R}_{f}=0.16$ (EtOAc/hexanes: 25/75); ${ }^{1} \mathrm{H}$ NMR $\left(\mathrm{CDCl}_{3}, 500 \mathrm{MHz}\right): \delta 7.89(\mathrm{dd}, J=8.0,1.0 \mathrm{~Hz}, 2 \mathrm{H}), 7.53(\mathrm{tt}, J$ = 7.0, $1.0 \mathrm{~Hz}, 1 \mathrm{H}), 7.42(\mathrm{t}, J=8.0 \mathrm{~Hz}, 2 \mathrm{H}), 5.71-5.59(\mathrm{~m}, 2 \mathrm{H}), 5.49(\mathrm{~s}, 1 \mathrm{H}), 5.07-4.96(\mathrm{~m}, 4 \mathrm{H})$, $4.66(\mathrm{~d}, J=32.0 \mathrm{~Hz}, 2 \mathrm{H}), 3.63(\mathrm{~s}, 3 \mathrm{H}), 2.45(\mathrm{dd}, J=14.0 \mathrm{~Hz}, 1 \mathrm{H}), 2.21-2.14(\mathrm{~m}, 2 \mathrm{H}), 2.09-1.97$ (m, 4H), 1.77-1.71 (m, 1H), 1.67-1.61 (m, 1H), 1.62 (s, 3H), 1.57-1.51 (m, 1H), $1.26(\mathrm{~s}, 3 \mathrm{H})$, $1.10(\mathrm{~s}, 3 \mathrm{H}) ;{ }^{13} \mathrm{C} \mathrm{NMR}\left(\mathrm{CDCl}_{3}, 126 \mathrm{MHz}\right) \delta 194.5,186.1,175.0,169.7,146.9,137.8,136.6,135.9$, 133.3, 129.4, 128.6, 126.5, 117.4, 116.5, 111.7, 103.2, 88.6, 55.5, 47.7, 44.7, 42.6, 42.5, 40.0, 36.2 $35.7,29.0,21.9,19.3$.

de Mayo Product 17: $1.4 \mathrm{mg}, 7 \% ; 4.0 \mathrm{mg}, 80 \%$ yield (from further treatment of $5.0 \mathrm{mg}$ of the $O$-cyclized byproduct 16); white solid, m. p.: 155-158 ${ }^{\circ} \mathrm{C}\left(\right.$ EtOAc/hexanes: 20/80); $\mathrm{R}_{f}=$ 0.27 (EtOAc/hexanes: 25/75); ${ }^{1} \mathrm{H}$ NMR $\left(\mathrm{CDCl}_{3}, 500 \mathrm{MHz}\right): \delta 7.87$ (d, $\left.J=7.5 \mathrm{~Hz}, 2 \mathrm{H}\right), 7.39$ (t, $J$ $=7.5 \mathrm{~Hz}, 1 \mathrm{H}), 7.30(\mathrm{t}, J=7.5 \mathrm{~Hz}, 2 \mathrm{H}), 5.79-5.70(\mathrm{~m}, 2 \mathrm{H}), 5.62(\mathrm{~s}, 1 \mathrm{H}), 5.10-4.96(\mathrm{~m}, 4 \mathrm{H}), 3.85$ (s, 3H), $2.86(\mathrm{~d}, J=12.5 \mathrm{~Hz}, 1 \mathrm{H}), 2.59(\mathrm{dd}, J=14.5,2.5 \mathrm{~Hz}, 1 \mathrm{H}), 2.23-2.18(\mathrm{~m}, 1 \mathrm{H}), 2.14-2.10$ (m, 2H), 1.94-1.88 (m, 1H), 1.82-1.76 (m, 1H), 1.62-1.54 (m, 3H), 1.32-1.25 (m, 1H), 1.21 (s, $3 \mathrm{H}), 1.15$ (t, $J=13.5 \mathrm{~Hz}, 1 \mathrm{H}), 0.92$ (s, 3H), 0.70 (s, 3H); $\left.{ }^{13} \mathrm{C} \mathrm{NMR} \mathrm{(CDCl} 3,126 \mathrm{MHz}\right): \delta 196.4$, 182.4, 138.2, 136.8, 136.5, 131.7, 129.4, 127.3, 116.4, 115.8, 101.7, 87.2, 75.0, 61.6, 56.7, 52.3,

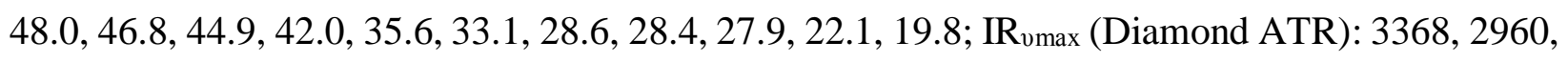
1712, 1655, 1608, 1361, 1221, 1205, 913, 809, $720 \mathrm{~cm}^{-1}$; HRMS (ESI): $[\mathrm{M}+\mathrm{Na}]^{+}$Calcd. For $\mathrm{C}_{30} \mathrm{H}_{36} \mathrm{O}_{4} \mathrm{Na}: 483.2511$, Found: 483.2493; $[\alpha]_{\mathrm{D}}^{23}=130.2\left(\mathrm{c}=0.100, \mathrm{CHCl}_{3}\right)$.

PPAP Core 23: $5.4 \mathrm{mg}, 27 \%$ yield; colorless oil; $\mathrm{R}_{f}=0.40$ (EtOAc/hexanes: 10/90); ${ }^{1} \mathrm{H}$ NMR ( $\left.\mathrm{CDCl}_{3}, 400 \mathrm{MHz}\right): \delta 7.43(\mathrm{dd}, J=7.2,1.2 \mathrm{~Hz}, 2 \mathrm{H}), 7.39(\mathrm{t}, J=7.2 \mathrm{~Hz}, 1 \mathrm{H}), 7.25(\mathrm{t}, J=7.6$ 
$\mathrm{Hz}, 2 \mathrm{H}), 5.90-5.74(\mathrm{~m}, 2 \mathrm{H}), 5.19-5.04(\mathrm{~m}, 4 \mathrm{H}), 4.12(\mathrm{~s}, 3 \mathrm{H}), 3.40(\mathrm{dd}, J=16.0,6.4 \mathrm{~Hz}, 1 \mathrm{H}), 3.26$ $(\mathrm{dd}, J=16.0,6.0 \mathrm{~Hz}, 1 \mathrm{H}), 2.74(\mathrm{dd}, J=14.0,6.4 \mathrm{~Hz}, 1 \mathrm{H}), 2.53(\mathrm{dd}, J=14.0,7.6 \mathrm{~Hz}, 1 \mathrm{H}), 1.93-$ $1.78(\mathrm{~m}, 3 \mathrm{H}), 1.35$ (d, $J=13.2 \mathrm{~Hz}, 1 \mathrm{H}), 1.27$ (s, 3H), 1.26 (s, 3H); ${ }^{13} \mathrm{C} \mathrm{NMR}\left(\mathrm{CDCl}_{3}, 101 \mathrm{MHz}\right)$ : $\delta$ 206.7, 193.6, 192.9, 172.2, 136.0, 135.6, 133.6, 132.0, 128.5, 127.9, 123.1, 118.7, 115.8, 78.9, 62.4, 59.4, 45.4, 36.7, 35.2, 34.6, 28.3, 25.8, 22.0; IRvmax (Diamond ATR): 2927, 1721, 1696, 1642, 1596, 1238, 1225, 917, $688 \mathrm{~cm}^{-1}$; HRMS (ESI): [M + H] ${ }^{+}$Calcd. For $\mathrm{C}_{25} \mathrm{H}_{29} \mathrm{O}_{4}: 393.2066$, Found: 393.2068 .

de Mayo Product 26: ${ }^{\mathrm{S}} 4.8 \mathrm{mg}$, 24\% yield; colorless oil; $\mathrm{R}_{f}=0.45$ (EtOAc/hexanes: 25/75); ${ }^{1} \mathrm{H}$ NMR (CDCl $\left.3,500 \mathrm{MHz}\right): \delta 7.46-7.41(\mathrm{~m}, 4 \mathrm{H}), 7.38-7.26(\mathrm{~m}, 6 \mathrm{H}), 5.93-5.79(\mathrm{~m}, 2 \mathrm{H})$, $5.64(\mathrm{~s}, 1 \mathrm{H}), 5.14(\mathrm{dd}, J=17.0,1.5 \mathrm{~Hz}, 1 \mathrm{H}), 5.09(\mathrm{~d}, J=10.0 \mathrm{~Hz}, 2 \mathrm{H}), 4.98(\mathrm{dd}, J=17.5,1.5 \mathrm{~Hz}$, $1 \mathrm{H}), 4.03(\mathrm{~s}, 3 \mathrm{H}), 3.61(\mathrm{~d}, J=9.0 \mathrm{~Hz}, 1 \mathrm{H}), 3.50(\mathrm{td}, J=9.5,4.5 \mathrm{~Hz}, 1 \mathrm{H}), 3.22$ (ddd, $J=31.516 .5$, $5.5 \mathrm{~Hz}, 2 \mathrm{H}), 2.64(\mathrm{dd}, J=14.5,7.0 \mathrm{~Hz}, 1 \mathrm{H}), 2.55(\mathrm{dd}, J=15.0,7.0 \mathrm{~Hz}, 1 \mathrm{H}), 2.31(\mathrm{dd}, J=13.5$, $4.5 \mathrm{~Hz}, 1 \mathrm{H}), 2.05(\mathrm{dd}, J=13.5,4.5 \mathrm{~Hz}, 1 \mathrm{H})$.

de Mayo Product 28: $2.6 \mathrm{mg}, 13 \%$ yield; beige amorphous solid; $\mathrm{R}_{f}=0.47$ (EtOAc/hexanes: 25/75); ${ }^{1} \mathrm{H}$ NMR (CDCl $\left.3,500 \mathrm{MHz}\right): \delta 7.40$ (d, $\left.J=7.0 \mathrm{~Hz}, 2 \mathrm{H}\right), 7.33$ (t, $J=7.0$ $\mathrm{Hz}, 2 \mathrm{H}), 7.27(\mathrm{t}, J=7.5 \mathrm{~Hz}, 1 \mathrm{H}), 5.93-5.78(\mathrm{~m}, 2 \mathrm{H}), 5.17-5.06(\mathrm{~m}, 4 \mathrm{H}), 4.98(\mathrm{dd}, J=17.0,1.5$ $\mathrm{Hz}, 1 \mathrm{H}$ ), 4.01 (s, 3H), 3.18 (ddd, $J=38.5,16.0,5.0 \mathrm{~Hz}, 2 \mathrm{H}), 3.04-3.01(\mathrm{~m}, 1 \mathrm{H}), 2.60$ (ddd, $J=$ 40.5, 14.5, $6.0 \mathrm{~Hz}, 2 \mathrm{H}), 2.07(\mathrm{dd}, J=13.5,5.0 \mathrm{~Hz}, 1 \mathrm{H}), 1.95(\mathrm{dd}, J=13.5,10.0 \mathrm{~Hz}, 1 \mathrm{H}), 1.41(\mathrm{~s}$, $3 \mathrm{H}), 0.81$ (s, 3H); ${ }^{13} \mathrm{C} \mathrm{NMR}\left(\mathrm{CDCl}_{3}, 126 \mathrm{MHz}\right): \delta 201.7,197.7,178.4,140.6,135.6,133.5,127.7$, 127.6, 127.5, 120.1, 118.9, 115.4, 83.3, 69.8, 63.8, 62.1, 48.7, 45.0, 32.7, 27.6, 27.2, 23.5, 21.3; IRvmax (Diamond ATR): 2917, 1760, 1639, 1582, 1447, 1333, 1234, 988, 920, $700 \mathrm{~cm}^{-1}$; HRMS (ESI): $[\mathrm{M}+\mathrm{H}]^{+}$Calcd. For $\mathrm{C}_{25} \mathrm{H}_{29} \mathrm{O}_{4}: 393.2066$, Found: 393.2067.

Rearomatized Product 29: $3.4 \mathrm{mg}, 17 \%$ yield; yellow oil; $\mathrm{R}_{f}=0.75$ (EtOAc/hexanes: 25/75); ${ }^{1} \mathrm{H}$ NMR (CDCl $\left.3,500 \mathrm{MHz}\right): \delta 11.09$ (s, $\left.1 \mathrm{H}\right), 7.73$ (dd, $\left.J=8.5,1.5 \mathrm{~Hz}, 2 \mathrm{H}\right), 7.53(\mathrm{tt}, J=$ 7.0, $1.5 \mathrm{~Hz}, 1 \mathrm{H}), 7.42$ (td, $J=8.0,0.5 \mathrm{~Hz}, 2 \mathrm{H}), 6.11-5.96$ (m, 2H), 5.09-4.94 (m, 4H), 4.59 (td, $J$ $=7.0,1.0 \mathrm{~Hz}, 1 \mathrm{H}), 3.93(\mathrm{~d}, J=7.5 \mathrm{~Hz}, 2 \mathrm{H}), 3.80(\mathrm{~s}, 3 \mathrm{H}), 3.46(\mathrm{dt}, J=6.0,1.5 \mathrm{~Hz}, 2 \mathrm{H}), 3.36(\mathrm{dt}$,

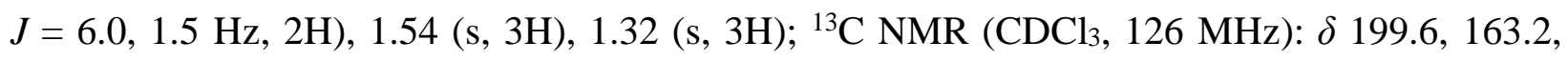
159.6, 157.3, 139.4, 138.1, 137.7, 136.6, 132.3, 129.6, 127.7, 119.2, 118.5, 117.3, 114.8, 114.5, 


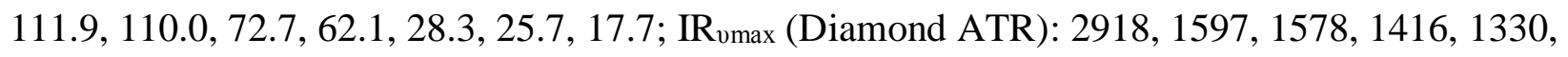
1282, 1118, $910 \mathrm{~cm}^{-1}$; HRMS (ESI): $[\mathrm{M}+\mathrm{Na}]^{+}$Calcd. For $\mathrm{C}_{25} \mathrm{H}_{28} \mathrm{O}_{4} \mathrm{Na}$ : 415.1885, Found: 415.1895 .

\section{Confirmation of the Type A Scaffolds}

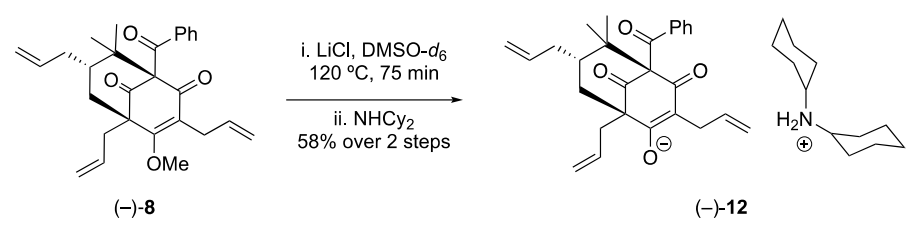

To a flame-dried 1-dram vial charged with argon and compound (-)-8 (5 mg, $11.6 \mu \mathrm{mol})$ was added deuterated DMSO (DMSO- $d_{6}, 0.2 \mathrm{~mL}$ ) and $\mathrm{LiCl}(10 \mathrm{mg}, 0.24 \mathrm{mmol}, 20$ equiv). The reaction was then heated to $120^{\circ} \mathrm{C}$ for $75 \mathrm{~min}$. After completion, the reaction was cooled to room temperature and to the mixture was added water $(5 \mathrm{~mL})$, followed by a mixture of diethyl ether and hexanes $(1: 1,10 \mathrm{~mL})$ and $1 \mathrm{~N} \mathrm{HCl}(5 \mathrm{~mL})$. The resulting mixture was extracted by diethyl ether and hexanes (1:1) $(10 \mathrm{~mL} \times 3)$, washed with water and brine, and dried over anhydrous $\mathrm{Na}_{2} \mathrm{SO}_{4}$. Solvent was removed in vacuo and the residue was purified by preparative plate chromatography (silica gel, EtOAc/hexanes/AcOH: 20:80:3) to afford the demethylated compound. To the demethylated compound was added diethyl ether $(2 \mathrm{~mL})$ and dicyclohexylamine (NHCy2, $3 \mu \mathrm{L}, 14 \mu \mathrm{mol}, 1.2$ equiv). The solution immediately turned bright yellow after the addition of the amine. The solvent was removed in vacuo and diethyl ether $(2 \mathrm{~mL})$ was added and removed in vасио. The resulting salt was recrystallized from chloroform and isooctane to afford a yellow solid; $4.1 \mathrm{mg}$, $58 \%$ yield; m. p.: $175-176^{\circ} \mathrm{C}$ (diethyl ether); $\mathrm{R}_{f}=0.44$ (EtOAc/hexanes: 50/50); ${ }^{1} \mathrm{H}$ NMR (CD $\left.3 \mathrm{OD}, 400 \mathrm{MHz}\right): \delta 7.76(\mathrm{dd}, J=8.4,1.2 \mathrm{~Hz}, 2 \mathrm{H}), 7.35(\mathrm{td}, J=8.0,1.2 \mathrm{~Hz}$, $1 \mathrm{H}), 7.20(\mathrm{td}, J=8.4,1.2 \mathrm{~Hz}, 2 \mathrm{H}), 5.89-5.73(\mathrm{~m}, 2 \mathrm{H}), 5.72-5.63(\mathrm{~m}, 1 \mathrm{H}), 5.04-4.90(\mathrm{~m}, 5 \mathrm{H}), 4.78$ (d, $J=10.0 \mathrm{~Hz}, 1 \mathrm{H}), 3.19-3.03(\mathrm{~m}, 4 \mathrm{H}), 2.54(\mathrm{~d}, J=13.6,6.4 \mathrm{~Hz}, 1 \mathrm{H}), 2.42(\mathrm{~d}, J=12.0,7.2 \mathrm{~Hz}$, 1H), 2.34-2.92 (m, 1H), 2.10-1.95 (m, 6H), 1.90-1.85 (m, 4H), 1.74-1.64 (m, 3H), 1.43-1.15 (m, $11 \mathrm{H}), 1.36(\mathrm{~s}, 3 \mathrm{H}), 1.05$ (s, 3H); ${ }^{13} \mathrm{C}$ NMR $\left(\mathrm{CD}_{3} \mathrm{OD}, 101 \mathrm{MHz}\right): \delta 212.3,196.3,187.9,185.6$, 138.1, 137.6, 125.4, 131.0, 128.3, 127.0, 116.0, 115.0, 114.8, 111.8, 78.1, 61.8, 53.1, 45.8, 41.6,

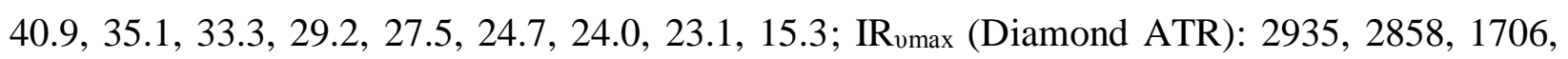


1690, 1488, 1386, 1223, 910, $689 \mathrm{~cm}^{-1}$; HRMS (ESI): $\left[\mathrm{M}^{-}+2 \mathrm{H}\right]^{+}$Calcd. For Anion $\mathrm{C}_{27} \mathrm{H}_{31} \mathrm{O}_{4}$ : 419.2222, Found: 419.2219; $[\alpha]_{\mathrm{D}}^{23}=-150.9\left(\mathrm{c}=0.100, \mathrm{CHCl}_{3}\right)$.
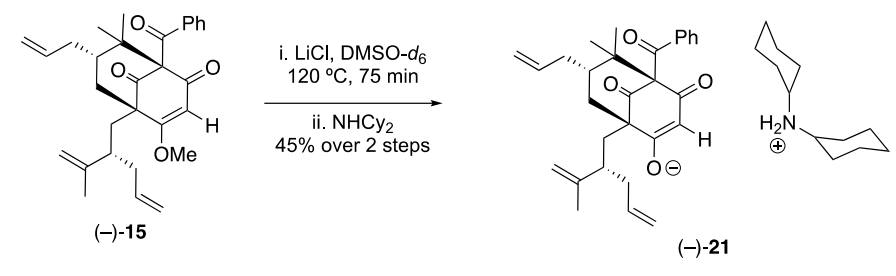

To a flame-dried 1-dram vial charged with argon and compound (-)-15 (11.7 mg, 25.4 $\mu \mathrm{mol})$ was added DMSO- $d_{6}(0.18 \mathrm{~mL})$ and $\mathrm{LiCl}(20 \mathrm{mg}, 0.47 \mathrm{mmol}, 18$ equiv). The reaction was then heated to $120^{\circ} \mathrm{C}$ for $75 \mathrm{~min}$. After completion, the reaction was cooled to room temperature and the mixture was added water $(10 \mathrm{~mL})$, diethyl ether and hexanes $(1: 1,20 \mathrm{~mL})$ and $1 \mathrm{~N} \mathrm{HCl}$ $(10 \mathrm{~mL})$. The resulting mixture was extracted by diethyl ether and hexanes $(1: 1)(10 \mathrm{~mL} \times 3)$, washed with water and brine, and dried over anhydrous $\mathrm{Na}_{2} \mathrm{SO}_{4}$. Solvent was removed in vacuo and the residue was purified by preparative plate chromatography (silica gel, EtOAc/hexanes/AcOH: 20:80:3) to afford the demethylated compound. To the demethylated compound was added diethyl ether $(2 \mathrm{~mL})$ and $\mathrm{NHCy}_{2}(6 \mu \mathrm{L}, 0.30 \mathrm{mmol}, 1.2$ equiv). The solvent was removed in vacuo and diethyl ether $(2 \mathrm{~mL})$ was added and removed in vacuo again. The resulting salt was recrystallized from chloroform and isooctane to afford a white solid; $7.2 \mathrm{mg}$, $45 \%$ yield; m. p.: $187-189^{\circ} \mathrm{C}$ (diethyl ether); $\mathrm{R}_{f}=0.10$ (EtOAc/hexanes: 50/50); ${ }^{1} \mathrm{H}$ NMR $\left(\mathrm{CDCl}_{3}\right.$, $500 \mathrm{MHz}): \delta 9.95(b r, 2 \mathrm{H}), 7.92(\mathrm{~d}, J=7.5 \mathrm{~Hz}, 2 \mathrm{H}), 7.38(\mathrm{tt}, J=7.0,1.0 \mathrm{~Hz}, 1 \mathrm{H}), 7.27(\mathrm{t}, J=8.0$ $\mathrm{Hz}, 2 \mathrm{H}), 5.64-5.52(\mathrm{~m}, 2 \mathrm{H}), 5.49(\mathrm{~s}, 1 \mathrm{H}), 4.98-4.84(\mathrm{~m}, 4 \mathrm{H}), 4.59(\mathrm{~d}, J=46.0 \mathrm{~Hz}, 2 \mathrm{H}), 2.60(b r$, 2H), 2.55-2.50 (m, 1H), 2.32-2.28 (m, 1H), 2.14-2.09 (m, 1H), 2.05-1.89 (m, 4H), 1,75-1.57 (m, $12 \mathrm{H}), 1.63(\mathrm{~s}, 3 \mathrm{H}), 1.39(\mathrm{~s}, 3 \mathrm{H}), 1.25-1.20(\mathrm{~m}, 5 \mathrm{H}), 1.12-0.99(\mathrm{~m}, 6 \mathrm{H}), 1.07$ (s, 3H); ${ }^{13} \mathrm{C} \mathrm{NMR}$ $\left(\mathrm{CDCl}_{3}, 126 \mathrm{MHz}\right): \delta 212.2,195.8,191.3,186.9,149.2,138.2,138.0,137.8,131.3,128.5,127.5$, 115.9, 114.5, 110.4, 104.3, 78.2, 61.2, 52.2, 46.6, 44.3, 43.3, 41.6, 38.9, 34.07, 34.01, 28.6, 28.3,

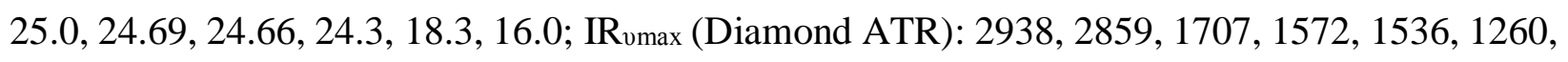
1053, $802 \mathrm{~cm}^{-1}$; HRMS (ESI): $\left[\mathrm{M}^{-}+2 \mathrm{H}\right]^{+}$Calcd. For Anion $\mathrm{C}_{29} \mathrm{H}_{35} \mathrm{O}_{4}: 447.2535$, Found: $447.2523 ;[\alpha]_{\mathrm{D}}^{25}=-188.2\left(\mathrm{c}=0.200, \mathrm{CHCl}_{3}\right)$. 


\section{Synthesis of (-)-Nemorosone}

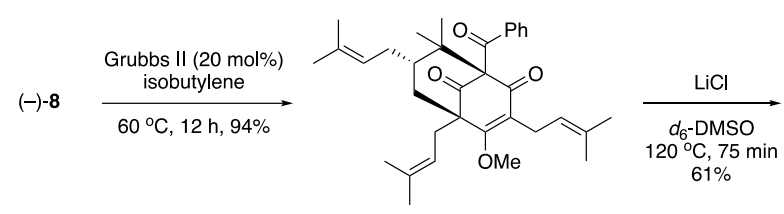

$(-)-13$

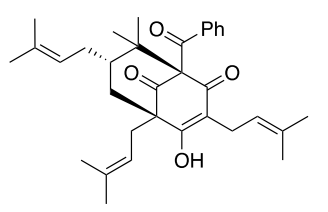

$2(-)$-nemorosone

$\boldsymbol{O}$-Methyl Nemorosone (-)-13: To a 15-mL glass pressure tube charged with argon was added compound $\mathbf{8}(18.8 \mathrm{mg}, 43.5 \mu \mathrm{mol})$ and Grubbs $2^{\text {nd }}$ generation catalyst $(7.2 \mathrm{mg}, 8.4 \mu \mathrm{mol})$. The tube was then cooled to $-78^{\circ} \mathrm{C}$ and isobutylene $(5.0 \mathrm{~mL})$ was added along the side of the tube. The tube was sealed and heated to $60^{\circ} \mathrm{C}$ for $12 \mathrm{~h}$. The reaction was then cooled back to $-78^{\circ} \mathrm{C}$ for $5 \mathrm{~min}$ and was opened to air for removal of isobutylene. The crude product was dissolved in $\mathrm{CH}_{2} \mathrm{Cl}_{2}(2.0 \mathrm{~mL})$ and purified by preparative plate chromatography (silica gel, EtOAc/hexanes: 10/90) to obtain 13 as a colorless oil. Spectroscopic data for $\mathbf{1 3}$ were found to be identical with those reported in the literature: ${ }^{\mathrm{S} 6} 21.2 \mathrm{mg}$, 94\% yield; $\mathrm{R}_{f}=0.51$ (EtOAc/hexanes: 20/80); ${ }^{1} \mathrm{H} \mathrm{NMR}$ $\left(\mathrm{CDCl}_{3}, 500 \mathrm{MHz}\right): \delta 7.44(\mathrm{dd}, J=7.5,1.0 \mathrm{~Hz}, 2 \mathrm{H}), 7.37(\mathrm{t}, J=7.5 \mathrm{~Hz}, 1 \mathrm{H}), 7.22(\mathrm{t}, J=7.5 \mathrm{~Hz}$, 2H), 5.04-4.99 (m, 3H), 4.00 (s, 3H), $3.25(\mathrm{dd}, J=15.0,7.0 \mathrm{~Hz}, 1 \mathrm{H}), 3.16(\mathrm{dd}, J=15.5,7.0 \mathrm{~Hz}$, $1 \mathrm{H}), 2.59(\mathrm{dd}, J=14.5,6.5 \mathrm{~Hz}, 1 \mathrm{H}), 2.43(\mathrm{dd}, J=14.0,6.0 \mathrm{~Hz}, 1 \mathrm{H}), 2.16(\mathrm{dd}, J=12.0,6.5 \mathrm{~Hz}$, $1 \mathrm{H}), 2.02(\mathrm{dd}, J=12.5,4.0 \mathrm{~Hz}, 1 \mathrm{H}), 1.76-1.67(\mathrm{~m}, 2 \mathrm{H}), 1.69$ (s, 3H), 1.68 (s, 3H), $1.65(\mathrm{~s}, 6 \mathrm{H})$, $1.63(\mathrm{~s}, 3 \mathrm{H}), 1.58(\mathrm{~s}, 3 \mathrm{H}), 1.47$ (dd, $J=13.5,12.0 \mathrm{~Hz}, 1 \mathrm{H}, \mathrm{CH} 2), 1.35$ (s, 3H), $1.13(\mathrm{~s}, 3 \mathrm{H}) ;{ }^{13} \mathrm{C}$ NMR ( $\left.\mathrm{CDCl}_{3}, 126 \mathrm{MHz}\right): \delta 207.4,194.2$, 193.2, 173.2, 136.4, 134.5, 133.6, 133.2, 132.0, 128.7, $127.9,126.9,122.7,121.5,119.7,79.4,62.6$, 60.0, 49.0, 43.9, 40.4, 29.9, 26.9, 26.2, 26.0, 25.8,

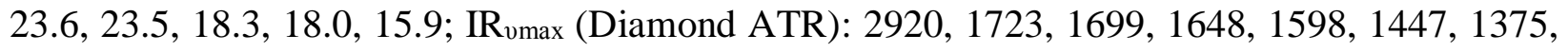
1237, 1066, 850, $806 \mathrm{~cm}^{-1}$; HRMS (ESI): $[\mathrm{M}+\mathrm{H}]^{+}$Calcd. For $\mathrm{C}_{34} \mathrm{H}_{45} \mathrm{O}_{4}$ : 517.3318, Found: 517.3300. $[\alpha]_{\mathrm{D}}^{22}=-50.2\left(\mathrm{c}=0.250, \mathrm{CHCl}_{3}\right)$.

(-)-Nemorosone 2: To a flame-dried 5-mL round-bottom flask charged with argon and 13 (12.3 mg, $21.3 \mu \mathrm{mol})$ was added anhydrous DMSO-d6 $(0.25 \mathrm{~mL}, 85 \mathrm{mM})$ and LiCl $(17 \mathrm{mg}, 0.39$ mmol, 18 equiv). The reaction was then heated to $120^{\circ} \mathrm{C}$ for $75 \mathrm{~min}$. After completion, the reaction was cooled down to room temperature and the mixture was added water $(2 \mathrm{~mL})$, diethyl ether and hexanes mixture $(1: 1,5 \mathrm{~mL})$ and $1 \mathrm{~N} \mathrm{HCl}(2 \mathrm{~mL})$. The resulting mixture was extracted by diethyl ether/hexanes mixture $(1: 1,5 \mathrm{~mL} \times 3)$, washed with water and brine and dried over anhydrous 
$\mathrm{Na}_{2} \mathrm{SO}_{4}$. Solvents were removed in vacuo and the residue was purified by preparative thin-layer chromatography (silica gel, EtOAc/hexanes/AcOH: 20/80/3) to afford natural product nemorosone as a slightly yellow oil. Spectroscopic data for $\mathbf{1 3}$ were found to be identical with those reported in the literature: ${ }^{\mathrm{S} 6} 7.3 \mathrm{mg}, 61 \%$ yield; $\mathrm{R}_{f}=0.47$ (EtOAc/hexanes: 40/60); ${ }^{1} \mathrm{H}$ NMR $\left(\mathrm{CD}_{3} \mathrm{OD}, 500\right.$ MHz): $\delta 7.54(\mathrm{dd}, J=7.0,0.8 \mathrm{~Hz}, 2 \mathrm{H}), 7.43(\mathrm{td}, J=7.5,1.0 \mathrm{~Hz}, 1 \mathrm{H}), 7.25(\mathrm{t}, J=8.0,0.5 \mathrm{~Hz}, 2 \mathrm{H})$, $5.08(\mathrm{tt}, J=7.5,1.0 \mathrm{~Hz}, 1 \mathrm{H}), 5.02-4.99(\mathrm{~m}, 2 \mathrm{H}), 3.10(\mathrm{ddd}, J=26.5,14.5,7.0 \mathrm{~Hz}, 2 \mathrm{H}), 2.50$ (ddd, $J=26.0,14.5,7.0 \mathrm{~Hz}, 2 \mathrm{H}), 2.18-2.13(\mathrm{~m}, 1 \mathrm{H}), 2.01(\mathrm{dd}, J=13.5,7.0 \mathrm{~Hz}, 1 \mathrm{H}), 1.75-1.69(\mathrm{~m}, 2 \mathrm{H})$, $1.69(\mathrm{~s}, 3 \mathrm{H}), 1.66(\mathrm{~s}, 3 \mathrm{H}), 1.65(\mathrm{~s}, 9 \mathrm{H}), 1.59(\mathrm{~s}, 3 \mathrm{H}), 1.44(\mathrm{t}, J=12.5 \mathrm{~Hz}, 1 \mathrm{H}), 1.34(\mathrm{~s}, 3 \mathrm{H}), 1.10$ (s, 3H); ${ }^{13} \mathrm{C}$ NMR (CD $\left.3 \mathrm{OD}, 126 \mathrm{MHz}\right): \delta 209.3$, 194.9, 138.2, 135.2, 134.2, 133.6, 133.1, 129.6, 128.8, 124.0, 122.2, 121.1, 120.8, 44.6, 42.3, 30.4, 28.2, 26.3, 26.04, 26.02, 24.3, 22.3, 18.3, 18.1,

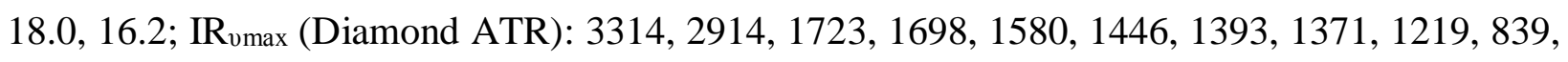
$687 \mathrm{~cm}^{-1}$; HRMS (ESI): $[\mathrm{M}+\mathrm{H}]^{+}$Calcd. For $\mathrm{C}_{33} \mathrm{H}_{43} \mathrm{O}_{4}: 503.3161$, Found: 503.3170; $[\alpha]_{\mathrm{D}}^{23}=-74.3$ $\left(\mathrm{c}=0.250, \mathrm{CHCl}_{3}\right)$.

\section{E. Synthesis of (-)-6-epi-Garcimultiflorone}

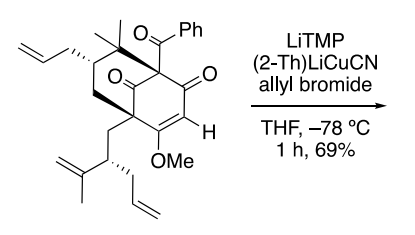

$(-)-15$

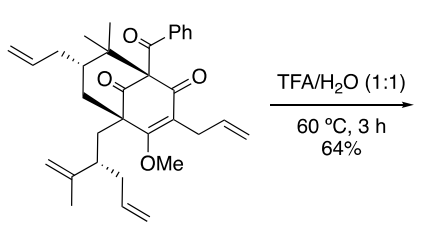

18

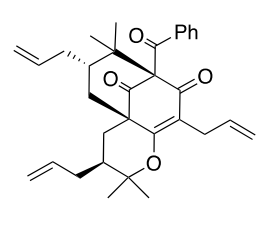

19

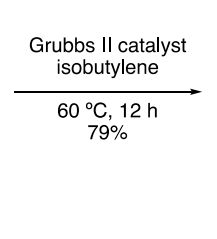

(-)-6-epi-garcimultiflorone A 20

Allyl derivative (-)-18 (lithium tetramethylpiperidine [LiTMP] preparation $^{\mathrm{S} 3}$ ): To a flamedried 10-mL round-bottom flask under an argon atmosphere containing a solution of 2,2,6,6tetramethylpiperidine $(420 \mu \mathrm{L}, 12.3 \mathrm{mmol})$ in THF $(4.8 \mathrm{~mL})$ at $-78{ }^{\circ} \mathrm{C}$ was added $n$-BuLi $(1 \mathrm{~mL}$, 2.5 $\mathrm{M}$ in hexanes) dropwise. The LiTMP solution was allowed to stir for $15 \mathrm{~min}$ at $-78^{\circ} \mathrm{C}$ prior to use. Then, to a flame-dried 25-mL round-bottom flask containing compound 15 (26.8 $\mathrm{mg}, 58.2$ $\mu \mathrm{mol})$ was added THF $(2.2 \mathrm{~mL})$ under argon. The solution was then cooled to $-78^{\circ} \mathrm{C}$. The freshly made LiTMP solution $(0.58 \mathrm{~mL}, 0.5 \mathrm{M}$ in THF) was added dropwise along the side of the flask which was followed by dropwise addition of lithium 2-thienylcyanocuprate $(1.3 \mathrm{~mL}, 0.25 \mathrm{M}$ in THF). Allyl bromide ( $30 \mu \mathrm{L}, 0.35 \mathrm{mmol}$ ) was then slowly added into the reaction mixture over the 
course of $1 \mathrm{~min}$. The reaction was allowed to stir for $1 \mathrm{~h}$ at $-78^{\circ} \mathrm{C}$. After completion, the mixture was warmed to room temperature and was quenched with $30 \% \mathrm{NH}_{4} \mathrm{OH}$ aqueous solution $(10 \mathrm{~mL})$, diluted with $\mathrm{Et}_{2} \mathrm{O}(10 \mathrm{~mL})$, and vigorously stirred for $5 \mathrm{~min}$. The mixture was poured into a separatory funnel and further extracted into $\mathrm{Et}_{2} \mathrm{O}(4 \mathrm{~mL} \times 3)$. The combined organic extracts were washed with water $(20 \mathrm{~mL})$ and brine $(20 \mathrm{~mL})$, dried over $\mathrm{Na}_{2} \mathrm{SO}_{4}$, filtered, and concentrated in vacuo. Purification by silica flash chromatography (EtOAc/hexanes: 0/100 to 10/90) provided the vinylic allylation product 18 as a slightly yellow oil. $20 \mathrm{mg}, 69 \%$ yield; $\mathrm{R}_{f}=0.29$ (EtOAc/hexanes: 10/90); ${ }^{1} \mathrm{H} \mathrm{NMR}\left(\mathrm{CDCl}_{3}, 500 \mathrm{MHz}\right): \delta 7.52$ (d, $\left.J=7.5 \mathrm{~Hz}, 2 \mathrm{H}\right), 7.40$ (t, $\left.J=7.5 \mathrm{~Hz}, 1 \mathrm{H}\right), 7.27$ (t, $J=8.0 \mathrm{~Hz}, 2 \mathrm{H}), 5.89-5.81(\mathrm{~m}, 1 \mathrm{H}), 5.67-5.55(\mathrm{~m}, 2 \mathrm{H}), 5.08-5.00(\mathrm{~m}, 4 \mathrm{H}), 4.92-4.89(\mathrm{~m}, 2 \mathrm{H})$, $4.67(\mathrm{~d}, J=33.5 \mathrm{~Hz}, 2 \mathrm{H}), 4.06(\mathrm{~s}, 3 \mathrm{H}), 3.35-3.24(\mathrm{~m}, 2 \mathrm{H}), 2.35-2.33(\mathrm{~m}, 2 \mathrm{H}), 2.15-2.12(\mathrm{~m}, 1 \mathrm{H})$, 2.05-2.02 (m, 2H), 2.00-1.95 (m, 1H), 1.87-1.84 (m, 1H), 1.79-1.70 (m, 2H), $1.66(\mathrm{~s}, 3 \mathrm{H}), 1.40$ (t, $J=12.0 \mathrm{~Hz}, 1 \mathrm{H}), 1.34(\mathrm{~s}, 3 \mathrm{H}), 1.11(\mathrm{~s}, 3 \mathrm{H}) ;{ }^{13} \mathrm{C} \mathrm{NMR}\left(\mathrm{CDCl}_{3}, 126 \mathrm{MHz}\right): \delta 207.7,193.4,192.8$, 173.4, 148.2, 137.0, 136.8, 136.1, 136.0, 132.0, 128.6, 127.9, 120.3, 116.9, 115.9, 115.3, 111.1, 79.3, 61.2, 59.4, 48.9, 44.7, 42.2, 41.9, 39.2, 34.6, 32.8, 28.6, 23.4, 17.5, 15.6; IR vmax (Diamond ATR): 2932, 1720, 1700, 1642, 1590, 1448, 1241, 1222, 994, $913 \mathrm{~cm}^{-1}$; HRMS (ESI): [M + H] $]^{+}$ Calcd. For $\mathrm{C}_{33} \mathrm{H}_{41} \mathrm{O}_{4}: 501.3005$, Found: 501.3008; $[\alpha]_{\mathrm{D}}^{24}=-138.2\left(\mathrm{c}=0.250, \mathrm{CHCl}_{3}\right)$.

$\boldsymbol{O}$-Cyclized Compound 19: To a scintillation vial containing 18 (9.0 mg, $0.018 \mathrm{mmol})$ was added a 1:1 mixture of water and TFA $(5.0 \mathrm{~mL})$. The resulting mixture was allowed to stir for $17 \mathrm{~h}$ before being concentrating in vacuo. The residue was purified by preparative thin-layer chromatography (EtOAc/hexanes: 10/90) to provide $O$-cyclized product 19 as a colorless oil. 5.6 mg, 64\% yield; $\mathrm{R}_{f}=0.28$ (EtOAc/hexanes: 10/90); ${ }^{1} \mathrm{H} \mathrm{NMR}\left(\mathrm{CDCl}_{3}, 500 \mathrm{MHz}\right): \delta 7.47$ (dd, $J=$ $8.5,1.0 \mathrm{~Hz}, 2 \mathrm{H}), 7.38(\mathrm{tt}, J=7.5,1.0 \mathrm{~Hz}, 1 \mathrm{H}), 7.24(\mathrm{td}, J=8.0,0.5 \mathrm{~Hz}, 2 \mathrm{H}), 5.78-5.60(\mathrm{~m}, 3 \mathrm{H})$, 5.12-4.99 (m, 5H), $4.92(\mathrm{dd}, J=10.0,2.0 \mathrm{~Hz}, 1 \mathrm{H}), 3.19-3.10(\mathrm{~m}, 2 \mathrm{H}), 2.87(\mathrm{dd}, J=14.5,3.5 \mathrm{~Hz}$, $1 \mathrm{H}), 2.41-2.37(\mathrm{~m}, 1 \mathrm{H}), 2.27-2.22(\mathrm{~m}, 1 \mathrm{H}), 2.07(\mathrm{dd}, J=13.5,4.5 \mathrm{~Hz}, 1 \mathrm{H}), 1.92-1.86(\mathrm{~m}, 1 \mathrm{H})$, $1.82-1.76(\mathrm{~m}, 1 \mathrm{H}), 1.75-1.69(\mathrm{~m}, 1 \mathrm{H}), 1.54-1.47(\mathrm{~m}, 2 \mathrm{H}), 1.46(\mathrm{~s}, 3 \mathrm{H}), 1.38(\mathrm{~s}, 3 \mathrm{H}), 1.34(\mathrm{~s}, 3 \mathrm{H})$,

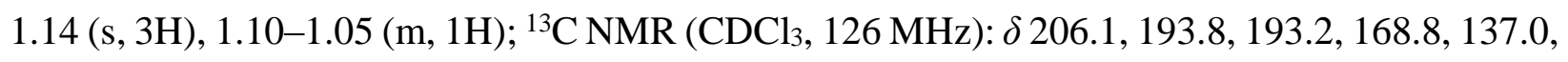
137.0, 135.7, 135.2, 132.1, 128.2, 128.1, 125.3, 117.4, 116.8, 115.8, 86.6, 78.8, 54.9, 47.7, 42.4, 41.9, 40.8, 35.5, 32.8, 29.6, 27.6, 27.4, 22.9, 22.0, 16.5; IR $v_{\max }$ (Diamond ATR): 2925, 1722, 1696, 
1640, 1598, 1371, 1346, 1226, $915 \mathrm{~cm}^{-1}$; HRMS (ESI): $[\mathrm{M}+\mathrm{H}]^{+}$Calcd. For $\mathrm{C}_{32} \mathrm{H}_{39} \mathrm{O}_{4}$ : 487.2848, Found: $487.2860 ;[\alpha]_{\mathrm{D}}^{24}=-219.5\left(\mathrm{c}=0.250, \mathrm{CHCl}_{3}\right)$.

(-)-6-Epi-Garcimultiflorone 20: To a flame-dried 30-mL glass pressure tube containing 19 (3.2 $\mathrm{mg}, 6.58 \mu \mathrm{mol})$ was added Grubbs catalyst $2^{\text {nd }}$ generation $(2.5 \mathrm{mg}, 1.94 \mu \mathrm{mol})$. The pressure tube was then purged with argon, cooled to $-78{ }^{\circ} \mathrm{C}$, and filled with isobutylene $(2 \mathrm{~mL})$. The reaction vessel was sealed and heated to $60^{\circ} \mathrm{C}$ with vigorous stirring. After $12 \mathrm{~h}$, the reaction was then cooled back to $-78^{\circ} \mathrm{C}$ for $5 \mathrm{~min}$ and was opened to the air to remove isobutylene. The residue was purified by preparative thin-layer chromatography (EtOAc/hexanes: 10/90) to provide (-)-6-epi-garcimultiflorone A 20 as a clear oil. $3.0 \mathrm{mg}$, 79\% yield; colorless oil; $\mathrm{R}_{f}=0.44$ (EtOAc/hexanes: 10/90); ${ }^{1} \mathrm{H}$ NMR $\left(\mathrm{CDCl}_{3}, 500 \mathrm{MHz}\right): \delta 7.49(\mathrm{~d}, J=8.0 \mathrm{~Hz}, 2 \mathrm{H}), 7.38(\mathrm{t}, J=7.5$ $\mathrm{Hz}, 1 \mathrm{H}), 7.24(\mathrm{t}, J=8.0 \mathrm{~Hz}, 2 \mathrm{H}), 5.11-5.08(\mathrm{~m}, 1 \mathrm{H}), 5.02-4.95(\mathrm{~m}, 2 \mathrm{H}), 3.13-3.03(\mathrm{~m}, 2 \mathrm{H}), 2.82$ $(\mathrm{dd}, J=14.0,3.5 \mathrm{~Hz}, 1 \mathrm{H}), 2.21-2.17(\mathrm{~m}, 1 \mathrm{H}), 2.10-2.08(\mathrm{~m}, 1 \mathrm{H}), 2.02(\mathrm{dd}, J=13.5,3.5 \mathrm{~Hz}, 1 \mathrm{H})$, 1.91-1.84 (m, 1H), 1.73-1.69 (m, 2H), 1.69 (s, 6H), 1.64 (s, 3H), 1.61 (s, 3H), 1.60 (s, 3H), 1.57 (s, 3H), 1.48-1.43 (m, 2H), $1.45(\mathrm{~s}, 3 \mathrm{H}), 1.39(\mathrm{~s}, 3 \mathrm{H}), 1.34$ (s, 3H), 1.15 (s, 3H), 1.04 (t, J = 13.0 $\mathrm{Hz}, 1 \mathrm{H}) ;{ }^{13} \mathrm{C} \mathrm{NMR}\left(\mathrm{CDCl}_{3}, 126 \mathrm{MHz}\right): \delta 206.4,194.0,193.5,168.2,137.1,133.8,133.3,132.2$, 132.0, 128.3, 128.0, 126.7, 122.7, 121.7, 121.4, 86.4, 78.9, 55.0, 47.9, 43.3, 42.2, 42.0, 29.7, 29.3, 27.7, 26.7, 25.9, 23.0, 22.1, 18.1, 18.0, 16.5; IR $\operatorname{vmax}$ (Diamond ATR): 2927, 1723, 1696, 1644, 1598, 1447, 1371, 1343, $1123 \mathrm{~cm}^{-1}$; HRMS (ESI): $[\mathrm{M}+\mathrm{H}]^{+}$Calcd. For $\mathrm{C}_{38} \mathrm{H}_{51} \mathrm{O}_{4}: 571.3787$, Found: 571.3806; $[\alpha]_{\mathrm{D}}^{24}=-190.6\left(\mathrm{c}=0.200, \mathrm{CHCl}_{3}\right)$.

\section{F. Derivatization of Dearomatized Compound 22}

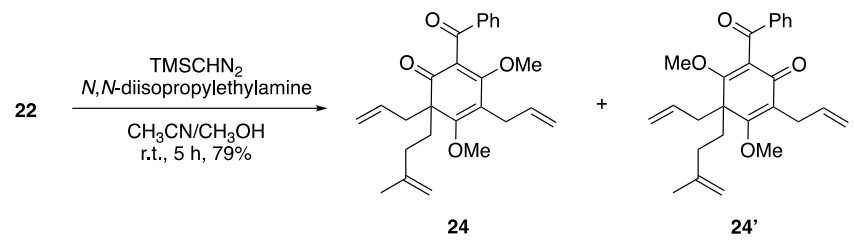

Methylation of 22: To a solution of dearomatized compound 22 (26 $\mathrm{mg}, 55 \mu \mathrm{mol})$ in acetonitrile and methanol $(9: 1,1 \mathrm{~mL})$ under argon was added $N, N$-diisopropylethylamine $(14 \mu \mathrm{L}$, $80 \mu \mathrm{mol})$ and trimethylsilyldiazomethane (TMSCHN $2,2 \mathrm{M}$ in hexanes, $0.12 \mathrm{mmol})$. The resulting 
mixture was stirred at room temperature for $5 \mathrm{~h}$ and was then quenched with $3 \mathrm{~N} \mathrm{HCl}$ solution and extracted with ethyl acetate $(10 \mathrm{~mL} \times 3)$. The combined organic layers were washed with brine, dried over anhydrous $\mathrm{Na}_{2} \mathrm{SO}_{4}$, filtered, and concentrated in vacuo. The residue was further purified by silica flash chromatography (EtOAc/hexanes: 5/95 to 10/90) to afford a pair of regioisomers (ratio $=1: 2)$ as a slightly yellow oil. $17.6 \mathrm{mg}, 79 \%$ yield; $\mathrm{R}_{f}=0.19\left(\right.$ EtOAc/hexanes: 10/90); ${ }^{1} \mathrm{H}$ NMR (2:1 mixture of diastereomers as determined by ${ }^{1} \mathrm{H} N \mathrm{NR}$ analysis, $\left.\mathrm{CDCl}_{3}, 400 \mathrm{MHz}\right): \delta$ $7.92-7.89(\mathrm{~m}, 2 \mathrm{H}), 7.52(\mathrm{t}, J=7.2 \mathrm{~Hz}, 1 \mathrm{H}), 7.43$ (t, $J=8.0 \mathrm{~Hz}, 2 \mathrm{H}), 6.02-5.83$ (m, 1.6H), 5.72$5.58(\mathrm{~m}, 1.4 \mathrm{H}), 5.13-4.98(\mathrm{~m}, 4.8 \mathrm{H}), 4.72-4.62(\mathrm{~m}, 2.6 \mathrm{H}), 3.98(\mathrm{~s}, 2 \mathrm{H}), 3.93(\mathrm{~s}, 1 \mathrm{H}), 3.68(\mathrm{~s}, 1 \mathrm{H})$, $3.64(\mathrm{~s}, 2 \mathrm{H}), 3.23-3.20(\mathrm{~m}, 2.4 \mathrm{H}), 2.74-2.56(\mathrm{~m}, 2.7 \mathrm{H}), 2.11-1.98(\mathrm{~m}, 2.2 \mathrm{H}), 1.96-1.80(\mathrm{~m}, 3 \mathrm{H})$, $1.72(\mathrm{~s}, 2 \mathrm{H}), 1.66(\mathrm{~s}, 1 \mathrm{H}) ;{ }^{13} \mathrm{C} \mathrm{NMR}\left(2: 1\right.$ mixture of regioisomers as determined by ${ }^{1} \mathrm{H} \mathrm{NMR}$ analysis, $\left.\mathrm{CDCl}_{3}, 101 \mathrm{MHz}\right): \delta 199.2,196.5,196.2,187.3,171.4,170.0,169.5,168.1,145.1,144.9$, 138.6, 138.3, 136.6, 136.3, 133.1, 133.02, 132.96, 129.3, 129.2, 128.6, 128.4, 121.3, 118.7, 118.3, 118.2, 117.9, 115.3, 115.0, 114.5, 110.1, 110.0, 63.1, 62.2, 60.7, 59.9, 59.0, 53.6, 44.1, 42.0, 37.3,

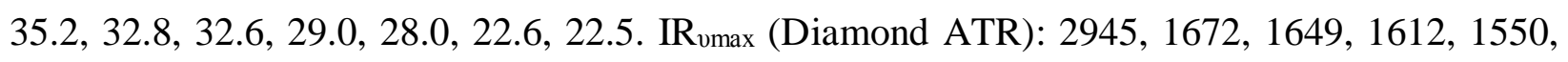
1449, 1362, 1220, 1129, 992, 736, $690632 \mathrm{~cm}^{-1}$; HRMS (ESI): $[\mathrm{M}+\mathrm{H}]^{+}$Calcd. For $\mathrm{C}_{26} \mathrm{H}_{31} \mathrm{O}_{4}$ : 407.2222, Found: 407.2227.

\section{G. Argon/Oxygen-Bubbling Experiments}

To a flame-dried vial was added dearomatized substrate $(20 \mathrm{mg})$ and anhydrous acetonitrile $(5 \mathrm{mM})$ and the mixture was degassed in sonication for $30 \mathrm{~min}$. After degassing, a 4-inch needle connected to a balloon (oxygen or argon) was inserted to the bottom of the vial. The reaction was then placed in front of the Kessil ${ }^{\circledR}$ LED lamp $(390 \mathrm{~nm})$ and another short needle was inserted to allow gas to bubble through the reaction mixture. The reactions were allowed to stir for $12 \mathrm{~h}$ at room temperature. Balloons were recharged if the gas ran out during the period. After reaction completion, the solvent was removed in vacuo and the residue was purified by preparative thinlayer chromatography (EtOAc/hexanes: 10/90). As indicated by the results listed below, for

dearomatized compound $\mathbf{5}$ the reactions showed no observable difference when bubbling with argon or oxygen. Meanwhile, for prenylated compound 27, oxygen completely hindered the reaction in a time period of $12 \mathrm{~h}$. 
(a)

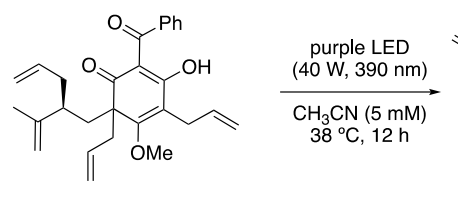

5

(b)

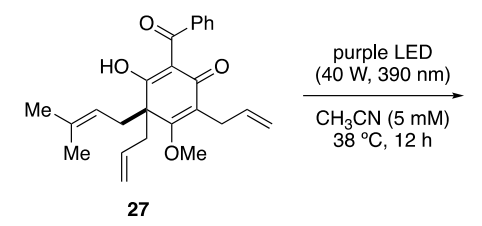

$\operatorname{argon}(24 \mathrm{~h})$ oxygen

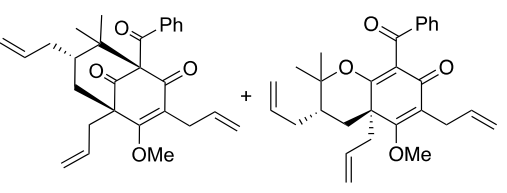

$(-)-8 \quad 11$

$3 \%$
$3 \%$

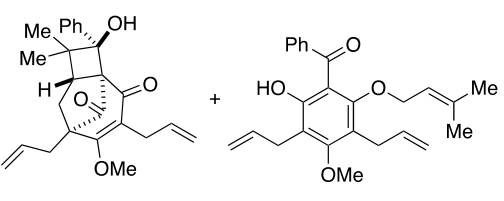

29

$17 \%$

\section{H. Deuterium-Labeling Experiment}

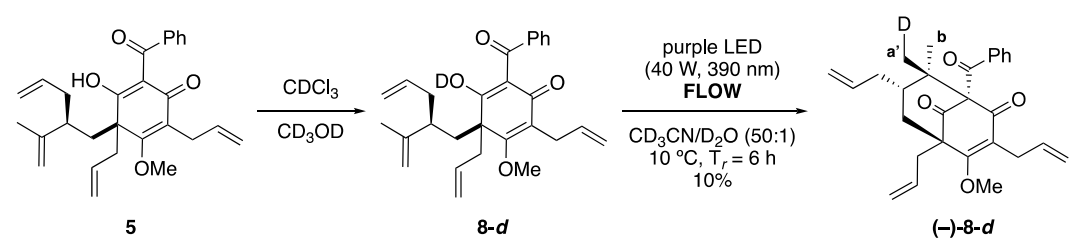

To a solution of $5(20 \mathrm{mg})$ in deuterated chloroform $\left(\mathrm{CDCl}_{3}, 2 \mathrm{~mL}\right)$ was added several drops of deuterated methanol $\left(\mathrm{CD}_{3} \mathrm{OD}\right)$. Place the mixture in sonication for $10 \mathrm{~min}$ and then remove all the solvent in vасио. The photoreaction was conducted with the resulting residue in flow photoreactor II (Procedure B) in a mixture of deuterated acetonitrile and deuterium oxide (CD $\left.{ }_{3} \mathrm{CN} / \mathrm{D}_{2} \mathrm{O}: 50 / 1,5 \mathrm{mM}\right)$. After completion, the solvent was removed in vacuo and the resulting residue was purified by preparative thin-layer chromatography (EtOAc/hexanes: 10/90), affording a mixture of (-)-8- $\boldsymbol{d}$ and (-)-8 (3.3:1, $2.0 \mathrm{mg}, 10 \%$ yield). To test the reversibility of deuteration, the non-deuterated product (-)-8 was treated under the same conditions. However, after work-up, the recovered product (-)-8 did not show any evidence of deuterium incorporation.

\section{Photophysical Studies}

Unless otherwise mentioned, whenever necessary spectrophotometric grade 2-methyl tetrahydrofuran (2-MeTHF) was used for photophysical experiments. UV/VIS absorbances were 
obtained with Agilent Technologies Cary 60 UV/VIS ${ }^{\circledR}$ instrument. Fluorescence emission, luminescence emission, excitation, phosphorescence emission, and lifetime experiments were carried out using Edinburgh instruments FLS 1000 ${ }^{\circledR}$ photoluminescence Spectrometer. A continuous wave Xenon lamp source was used for recording fluorescence, luminescence at $77 \mathrm{~K}$, and excitation spectra. A microsecond-pulsed Xenon lamp source was used to record phosphorescence spectra and phosphorescence lifetimes. Fluorescence, luminescence, phosphorescence and excitation spectra were reported as the average of three scans with a dwell time of $0.2 \mathrm{~s}$.

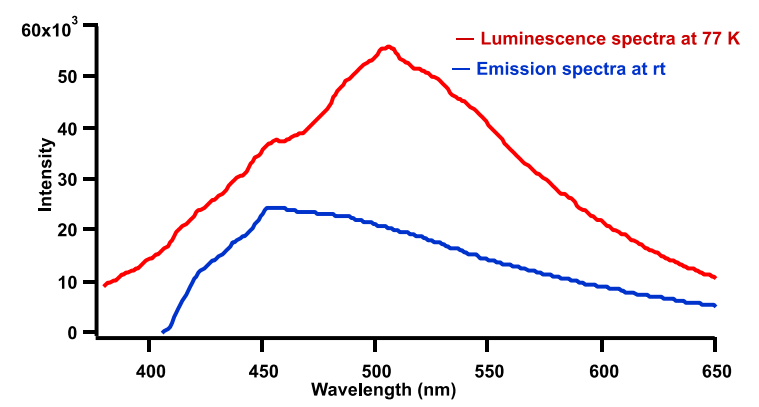

Figure S4. Luminescence of 5 at $77 \mathrm{~K}$ (red) and its room temperature fluorescence (blue) in 2-MeTHF.
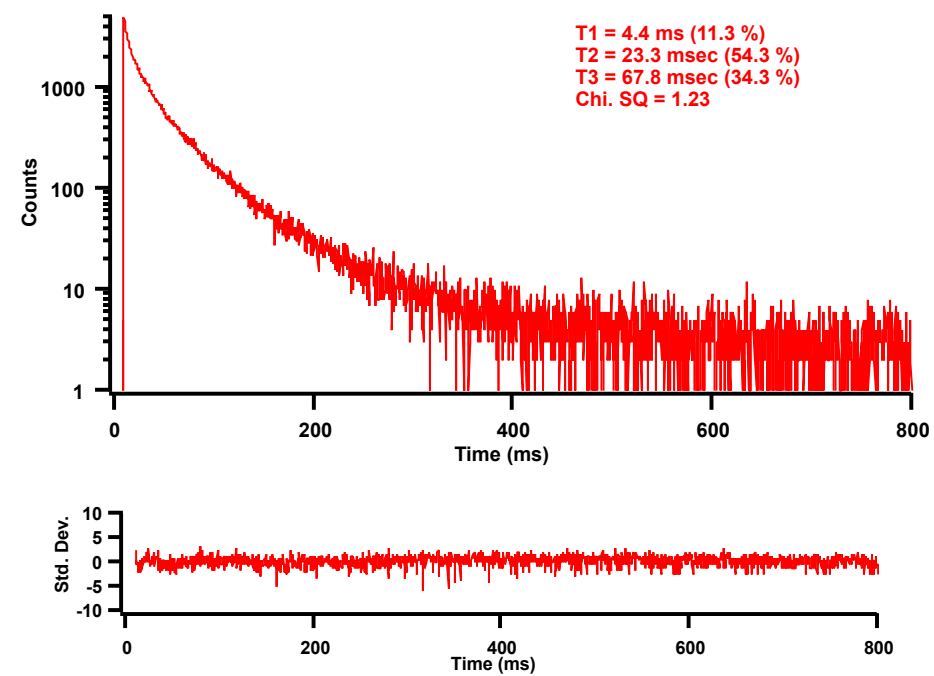

Figure S5. Phosphorescence lifetime decay of compound 5 in 2-MeTHF glass at $77 \mathrm{~K}$. ( $\tau_{1}=4.4 \mathrm{~ms}, \tau_{2}=23.3 \mathrm{~ms}$ and $\left.\tau_{3}=67.8 \mathrm{~ms}\right)$.

Photophysical studies of compounds 5 and 25. For photophysical studies of compounds 5 and 25, $30 \mu \mathrm{M}$ solution in 2-MeTHF was used. Concentration was adjusted to have optical density (O.D) 0.15 at the excitation wavelength. Normalized UV spectra of compound $\mathbf{5}$ matched 
with its normalized excitation spectra (Figure $\mathbf{2}$ for compound $\mathbf{5}$ and Figure $\mathbf{S} \mathbf{4}$ for compound 25) indicating that the compounds are optically pure for photophysical experiments. For compound $\mathbf{5}$, UV/VIS absorption spectra and excitation spectra were recorded from $300 \mathrm{~nm}$ to $415 \mathrm{~nm}$ that showed maxima around $354 \mathrm{~nm}$ (Figure 2). Fluorescence was recorded with excitation and emission arm slit width of $6 \mathrm{~nm}$ by excitation at $350 \mathrm{~nm}$. The fluorescence signal had maximum intensity at $454 \mathrm{~nm}$ (Figure S4). Excitation spectra were monitored at room temperature from the fluorescence signal at $454 \mathrm{~nm}$ with an excitation and emission arm slit width of $6 \mathrm{~nm}$. Phosphorescence spectra were recorded at $77 \mathrm{~K}$ with an excitation and emission arm slit width of $6 \mathrm{~nm}$ and $4 \mathrm{~nm}$, respectively. Phosphorescence spectra had maximum intensity around $510 \mathrm{~nm}$ corresponding to a triplet energy $\sim 56 \mathrm{kcal} / \mathrm{mol}$. Luminescence spectra were recorded at $77 \mathrm{~K}$ using a continuous wave Xenon lamp source without time gating to record the signal from both fluorescence and phosphorescence. Excitation and emission arm slit widths were used to record luminescence spectra at $77 \mathrm{~K}$ and were $1 \mathrm{~nm}$ and $4 \mathrm{~nm}$, respectively.

The phosphorescence emission lifetime for compound $\mathbf{5}$ was determined using a microsecond pulsed Xenon lamp source of $1.3 \mathrm{~Hz}$ with detector gating delay of $9 \mathrm{~ms}$ and gate width of $790 \mathrm{~ms}$ (Figure S5). Tri-exponential decay was observed with lifetimes of $\tau_{1}=4.4 \mathrm{~ms}, \tau_{2}$ $=23.3 \mathrm{~ms}$, and $\tau_{3}=67.8 \mathrm{~ms} . \chi^{2}=1.23$.

For compound 25 (Figure S6), UV/VIS absorption spectra and excitation spectra were monitored from $250 \mathrm{~nm}$ to $420 \mathrm{~nm}$ with a maximum around $352 \mathrm{~nm}$. Fluorescence spectra were recorded with an excitation and emission arm slit width of $5 \mathrm{~nm}$ by excitation at $375 \mathrm{~nm}$. The fluorescence signal had a maximum intensity around $450 \mathrm{~nm}$. Excitation spectra were monitored from the fluorescence signal at room temperature with a slit width of $5 \mathrm{~nm}$ at both the excitation and emission arms. Phosphorescence spectra were recorded at $77 \mathrm{~K}$ with an excitation and emission arm slit width of $5 \mathrm{~nm}$ with a detector gating delay of $0.2 \mathrm{~ms}$. Phosphorescence spectra had maximum intensity around $536 \mathrm{~nm}$ corresponding to the triplet energy of $53.3 \mathrm{kcal} / \mathrm{mol}$. Luminescence spectra were recorded at $77 \mathrm{~K}$ using continuous wave Xenon lamp source without time gating to record the signal from fluorescence and phosphorescence. 


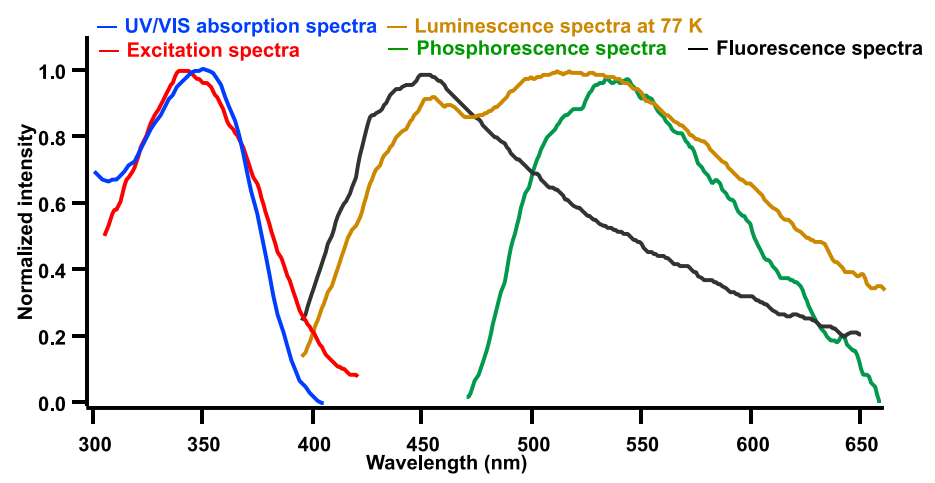

Figure S6. UV/VIS absorption (blue), excitation (red), fluorescence (black), phosphorescence (green) and emission spectra at $77 \mathrm{~K}$ (orange) of compound 25 in 2-MeTHF. UV/VIS absorption, fluorescence and excitation spectra were recorded at room temperature. Phosphorescence spectra and emission spectra were recorded at $77 \mathrm{~K}$ in $2-\mathrm{MeTHF}$ glass.

The phosphorescence emission lifetime for compound 25 was determined using a microsecond pulsed Xenon lamp source of $0.1 \mathrm{~Hz}$ with a detector gating delay of $1 \mathrm{~ms}$ and gate width of $10 \mathrm{~s}$ (Figure S7). Bi-exponential decay was observed with a lifetime of $\tau_{1}=9.4 \mathrm{~ms}$ and $\tau_{2}$ $=40.5 \mathrm{~ms} \cdot \chi^{2}=1.07$.
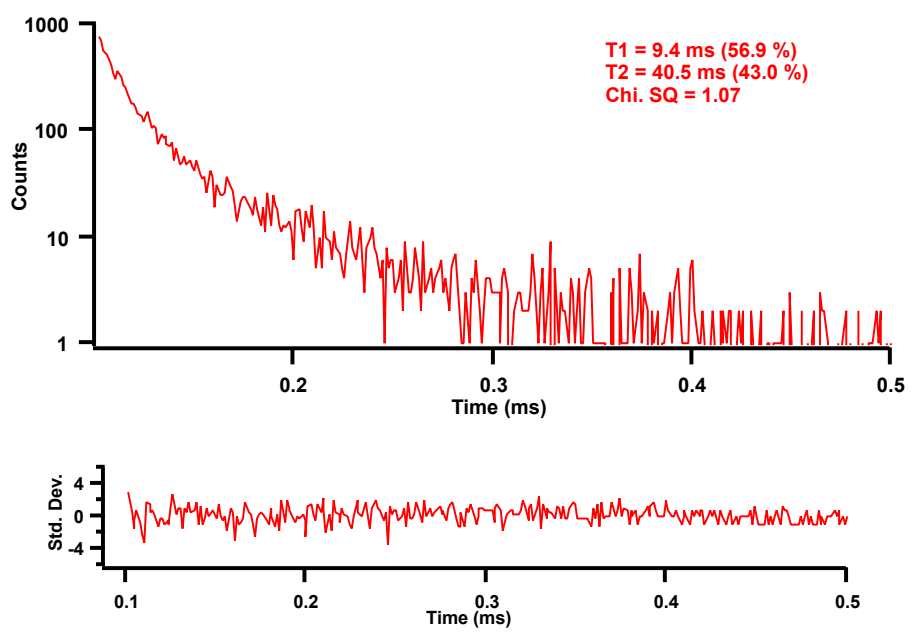

Figure S7. Phosphorescence lifetime decay of compound 25 in 2 -MeTHF glass at 77 K. $\left(\tau_{1}=9.4 \mathrm{~ms}\right.$ and $\tau_{2}=40.5$ $\mathrm{ms})$. 


\section{X-ray Crystallographic Data}

\section{A. Compound 12}

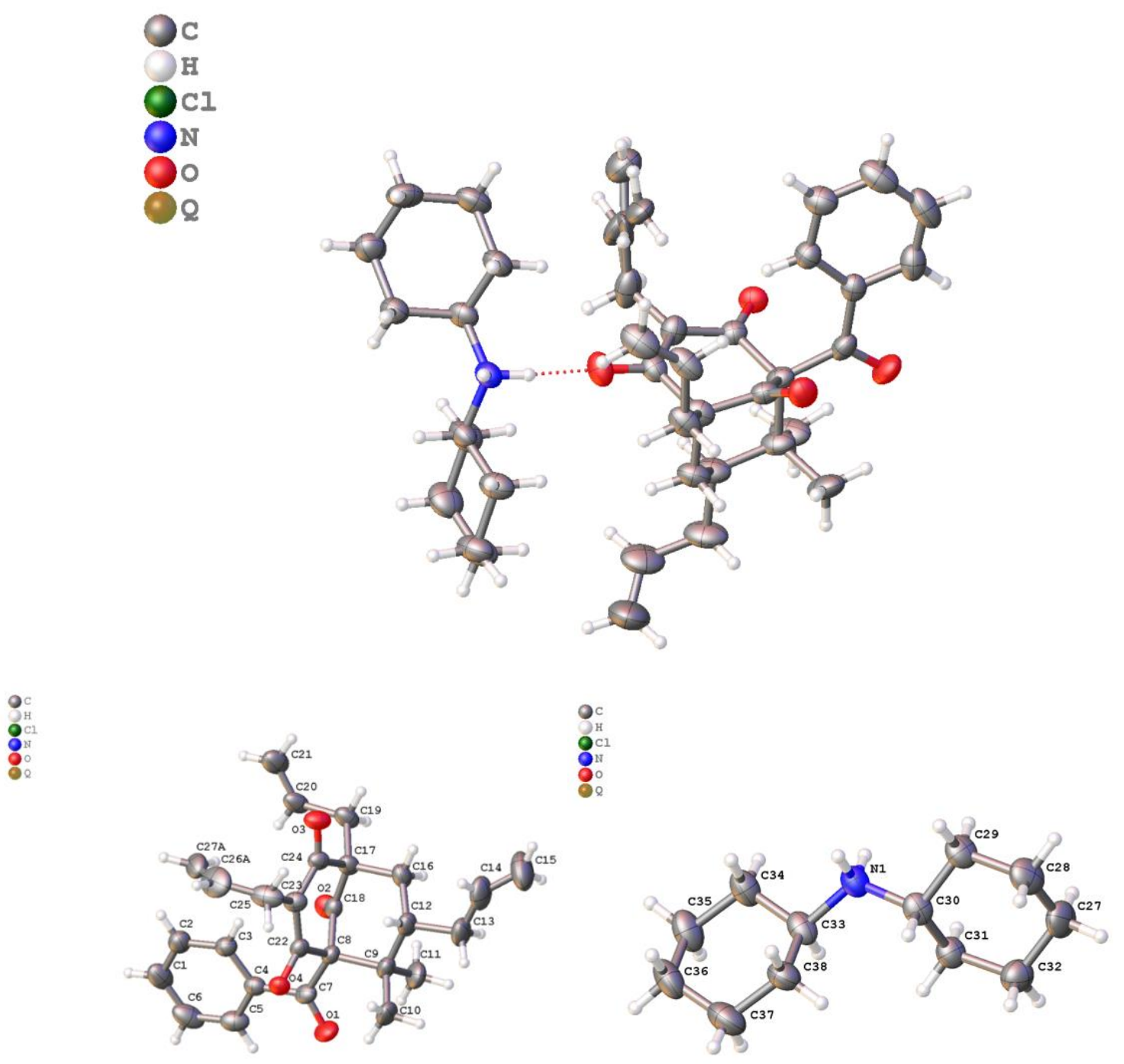

Crystals of compound 12 suitable for X-ray analysis were obtained by layering a $\mathrm{CHCl}_{3}$ solution with isooctane at ambient temperature followed by storage in a sealed vial. Crystallographic data have been deposited with the Cambridge Crystallographic Data Centre (CCDC \# 1913293). Copies of the data can be obtained free of charge through application to the 
CCDC, 12 Union Road, Cambridge CB21EZ, UK (fax: (+44)-1223-336-033; e-mail: deposit@ccdc.cam.ac.uk).

\section{Computing details}

Cell refinement: SAINT V8.38A (Bruker, 2016); data reduction: SAINT V8.38A (Bruker, 2016); program(s) used to solve structure: ShelXT (Sheldrick, 2015); program(s) used to refine structure: SHELXL (Sheldrick, 2015); molecular graphics: Olex2 (Dolomanov et al., 2009); software used to prepare material for publication: Olex2 (Dolomanov et al., 2009).

Table S2. Crystal data.

\begin{tabular}{|l|l|}
\hline $\mathrm{C}_{27} \mathrm{H}_{29} \mathrm{O}_{4} \cdot \mathrm{C}_{12} \mathrm{H}_{24} \mathrm{~N} \cdot 0.87\left(\mathrm{CHCl}_{3}\right)$ & $F(000)=753$ \\
\hline$M_{r}=704.09$ & $D_{\mathrm{x}}=1.208 \mathrm{Mg} \mathrm{m}^{-3}$ \\
\hline Monoclinic, $P 2_{1}$ & Cu $K \alpha$ radiation, $\lambda=1.54178 \AA$ \\
\hline$a=10.3691(5) \AA$ & Cell parameters from 9447 reflections \\
\hline$b=17.1035(8) \AA$ & $\theta=4.1-66.4^{\circ}$ \\
\hline$c=11.0229(5) \AA$ & $\mu=2.21 \mathrm{~mm}^{-1}$ \\
\hline$\beta=97.966(2)^{\circ}$ & $T=296 \mathrm{~K}$ \\
\hline$V=1936.02(16) \AA^{3}$ & Block, colourless \\
\hline$Z=2$ & $0.38 \times 0.12 \times 0.08 \mathrm{~mm}$ \\
\hline
\end{tabular}

Table S3. Data collection.

\begin{tabular}{|l|l|}
\hline Bruker APEX-II CCD diffractometer & 6555 reflections with $I>2 \sigma(I)$ \\
\hline$\phi$ and $\omega$ scans & $R_{\text {int }}=0.051$ \\
\hline $\begin{array}{l}\text { Absorption correction: multi-scan } \\
S A D A B S 2016 / 2 \text { (Bruker,2016/2) was used for }\end{array}$ & $\theta_{\max }=67.2^{\circ}, \theta_{\min }=4.1^{\circ}$ \\
\hline
\end{tabular}




\begin{tabular}{|l|l|}
\hline $\begin{array}{l}\text { absorption correction. wR2(int) was } 0.1286 \\
\text { before and } 0.0812 \text { after correction. The Ratio of } \\
\text { minimum to maximum transmission is } 0.7824 .\end{array}$ \\
The $\lambda / 2$ correction factor is Not present. & \\
\hline$T_{\min }=0.589, T_{\max }=0.753$ & $h=-12 \varnothing 12$ \\
\hline 64642 measured reflections & $k=-20 \varnothing 20$ \\
\hline 6845 independent reflections & $l=-13 \varnothing 12$ \\
\hline
\end{tabular}

\section{Table S4. Refinement parameters.}

\begin{tabular}{|l|l|}
\hline Refinement on $F^{2}$ & $\begin{array}{l}\text { Hydrogen site location: inferred from } \\
\text { neighbouring sites }\end{array}$ \\
\hline Least-squares matrix: full & H-atom parameters constrained \\
\hline$R\left[F^{2}>2 \sigma\left(F^{2}\right)\right]=0.042$ & $w=1 /\left[\sigma^{2}\left(F_{\mathrm{o}}^{2}\right)+(0.077 P)^{2}+0.376 P\right]$ \\
\hline$w R\left(F^{2}\right)=0.119$ & where $P=\left(F_{\mathrm{o}}{ }^{2}+2 F_{\mathrm{c}}^{2}\right) / 3$ \\
\hline$S=1.04$ & $(\Delta / \sigma)_{\max }<0.001$ \\
\hline 6845 reflections & $\Delta\rangle_{\max }=0.37$ e $\AA^{-3}$ \\
\hline 515 parameters & $\Delta\rangle_{\min }=-0.18$ e $\AA^{-3}$ \\
\hline 807 restraints & Absolute structure: Flack x determined using \\
\hline Primary atom site location: dual & 3008 quotients [(I+)-(I-)]/[(I+)+(I-)] (Parsons, \\
& Flack and Wagner, Acta Cryst. B69 (2013) 249- \\
\hline & $259)$. \\
\hline
\end{tabular}




\section{Special details}

Geometry. All esds (except the esd in the dihedral angle between two 1.s. planes) are estimated using the full covariance matrix. The cell esds are taken into account individually in the estimation of esds in distances, angles and torsion angles; correlations between esds in cell parameters are only used when they are defined by crystal symmetry. An approximate (isotropic) treatment of cell esds is used for estimating esds involving l.s. planes.

Table S5. Fractional atomic coordinates and isotropic or equivalent isotropic displacement parameters $\left(\AA^{2}\right)$ for 12.

\begin{tabular}{|l|l|l|l|l|l|}
\hline & $x$ & $y$ & $z$ & $U_{\text {iso }} * U_{\text {eq }}$ & Occ. $(<1)$ \\
\hline O4 & $0.3074(2)$ & $0.21222(11)$ & $0.40127(18)$ & $0.0358(4)$ & \\
\hline O2 & $0.07159(19)$ & $0.43266(12)$ & $0.3732(2)$ & $0.0438(5)$ & \\
\hline O3 & $0.5114(2)$ & $0.45709(13)$ & $0.4206(2)$ & $0.0458(5)$ & \\
\hline O1 & $-0.0094(2)$ & $0.23884(15)$ & $0.2827(2)$ & $0.0522(6)$ & \\
\hline N1 & $0.7017(2)$ & $0.56622(14)$ & $0.4807(2)$ & $0.0375(5)$ & \\
\hline H1A & 0.6880 & 0.6121 & 0.5149 & $0.045^{*}$ & \\
\hline H1B & 0.6250 & 0.5420 & 0.4665 & $0.045^{*}$ & \\
\hline C22 & $0.3100(3)$ & $0.28452(16)$ & $0.3822(2)$ & $0.0317(6)$ & \\
\hline C24 & $0.4141(3)$ & $0.41435(16)$ & $0.3954(3)$ & $0.0347(6)$ & \\
\hline C4 & $0.0514(3)$ & $0.27323(16)$ & $0.4877(3)$ & $0.0344(6)$ & \\
\hline C18 & $0.1715(3)$ & $0.40534(16)$ & $0.3460(2)$ & $0.0338(6)$ & \\
\hline C3 & $0.1380(3)$ & $0.30661(17)$ & $0.5811(3)$ & $0.0373(6)$ & \\
\hline H3 & 0.2124 & 0.3316 & 0.5622 & $0.045^{*}$ & \\
\hline
\end{tabular}




\begin{tabular}{|c|c|c|c|c|}
\hline C 30 & 0.7878 & $0.51884(16)$ & 0.5735 (3) & $0.0391(6)$ \\
\hline H30 & 0.7982 & 0.4666 & 0.5396 & $0.047 *$ \\
\hline $\mathrm{C} 7$ & 0.0687 (3) & $0.27395(17)$ & $0.3550(3)$ & $0.0377(6)$ \\
\hline $\mathrm{C} 17$ & $0.2893(3)$ & $0.45266(16)$ & $0.3313(3)$ & $0.0369(6)$ \\
\hline $\mathrm{C} 23$ & $0.4171(3)$ & $0.33331(17)$ & $0.4186(3)$ & $0.0381(6)$ \\
\hline $\mathrm{C} 5$ & $-0.0584(3)$ & $0.23531(19)$ & $0.5206(3)$ & 0.0479 (7) \\
\hline H5 & -0.1176 & 0.2120 & 0.4602 & $0.057^{*}$ \\
\hline C31 & $0.9213(3)$ & $0.55567(18)$ & $0.6044(3)$ & $0.0416(7)$ \\
\hline $\mathrm{H} 31 \mathrm{~A}$ & 0.9119 & 0.6093 & 0.6306 & $0.050^{*}$ \\
\hline $\mathrm{H} 31 \mathrm{~B}$ & 0.9645 & 0.5565 & 0.5318 & $0.050 *$ \\
\hline $\mathrm{C} 20$ & $0.2744(4)$ & $0.54438(17)$ & 0.5108 (3) & $0.0481(8)$ \\
\hline $\mathrm{H} 20$ & 0.2090 & 0.5177 & 0.5433 & $0.058 *$ \\
\hline C33 & $0.7456(3)$ & $0.58264(19)$ & 0.3579 (3) & $0.0422(7)$ \\
\hline H33 & 0.8250 & 0.6144 & 0.3713 & $0.051 *$ \\
\hline C9 & $0.1851(4)$ & $0.31796(18)$ & 0.1677 (3) & $0.0450(7)$ \\
\hline C38 & $0.7753(3)$ & $0.5076(2)$ & $0.2944(3)$ & $0.0480(7)$ \\
\hline H38A & 0.7002 & 0.4732 & 0.2874 & $0.058^{*}$ \\
\hline H38B & 0.8479 & 0.4810 & 0.3423 & $0.058 *$ \\
\hline $\mathrm{C} 8$ & 0.1834 (3) & $0.31967(15)$ & 0.3135 (2) & $0.0340(6)$ \\
\hline $\mathrm{C} 2$ & $0.1150(3)$ & $0.3032(2)$ & $0.7010(3)$ & $0.0480(7)$ \\
\hline $\mathrm{H} 2$ & 0.1738 & 0.3263 & 0.7620 & $0.058^{*}$ \\
\hline C19 & 0.2764 (3) & $0.53743(17)$ & 0.3755 (3) & $0.0443(7)$ \\
\hline
\end{tabular}




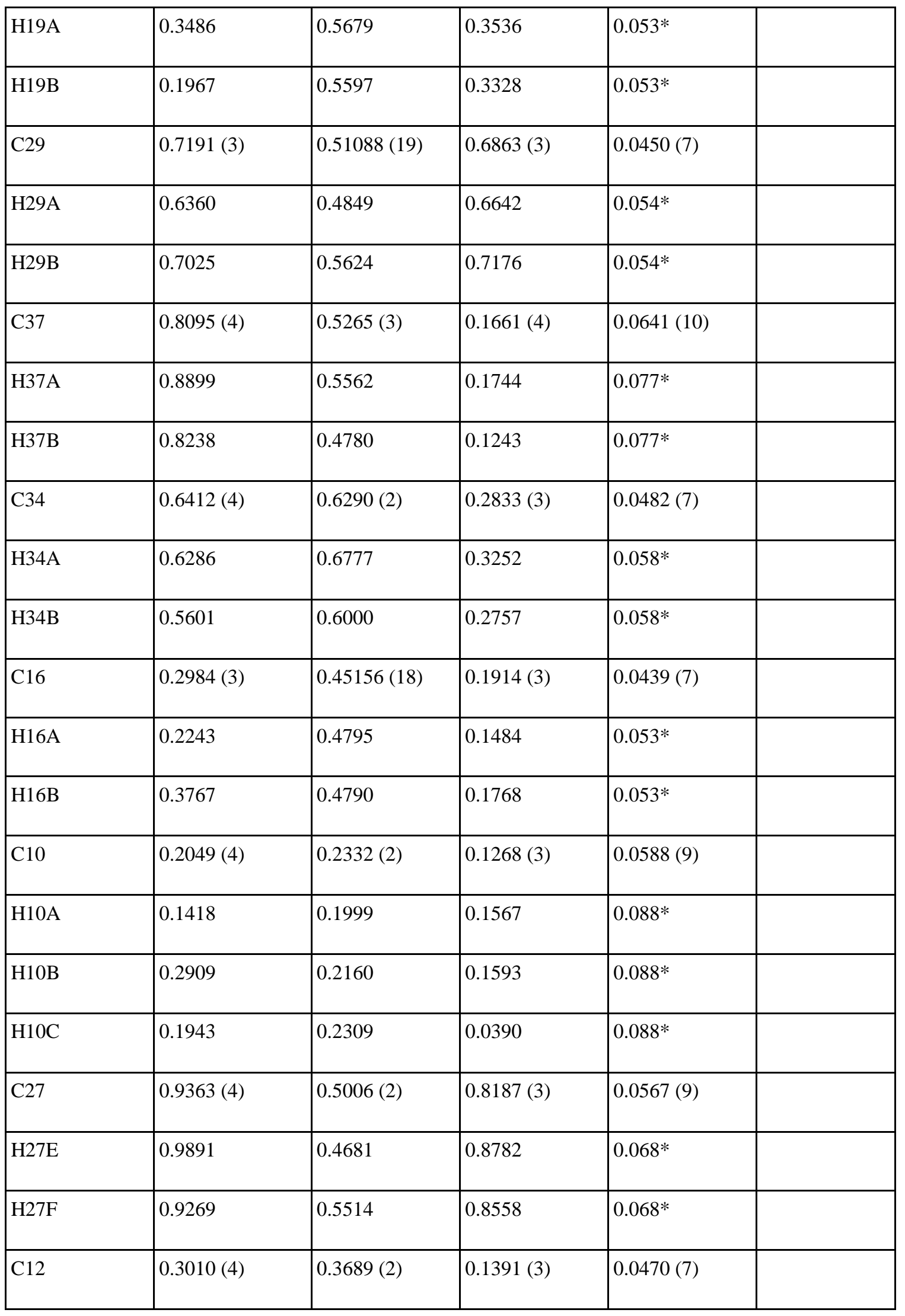




\begin{tabular}{|c|c|c|c|c|c|}
\hline H12 & 0.3811 & 0.3438 & 0.1781 & $0.056^{*}$ & \\
\hline $\mathrm{C} 32$ & $1.0046(3)$ & $0.5103(2)$ & 0.7058 & $0.0522(8)$ & \\
\hline $\mathrm{H} 32 \mathrm{~A}$ & 1.0242 & 0.4591 & 0.6752 & $0.063 *$ & \\
\hline H32B & 1.0863 & 0.5377 & 0.7285 & $0.063^{*}$ & \\
\hline $\mathrm{C} 1$ & 0.0068 (4) & $0.2663(2)$ & $0.7320(4)$ & $0.0568(9)$ & \\
\hline H1 & -0.0080 & 0.2643 & 0.8132 & $0.068^{*}$ & \\
\hline $\mathrm{C} 25$ & 0.5417 (3) & $0.2976(2)$ & 0.4794 (4) & 0.0584 (9) & \\
\hline $\mathrm{H} 25 \mathrm{~A}$ & 0.5450 & 0.2440 & 0.4511 & $0.070 *$ & $0.755(13)$ \\
\hline $\mathrm{H} 25 \mathrm{~B}$ & 0.6134 & 0.3254 & 0.4511 & $0.070 *$ & $0.755(13)$ \\
\hline $\mathrm{H} 25 \mathrm{C}$ & 0.5454 & 0.2430 & 0.4564 & $0.070 *$ & $0.245(13)$ \\
\hline $\mathrm{H} 25 \mathrm{D}$ & 0.6153 & 0.3243 & 0.4526 & $0.070 *$ & $0.245(13)$ \\
\hline C6 & $-0.0801(4)$ & $0.2321(2)$ & $0.6404(4)$ & $0.0608(10)$ & \\
\hline H6 & $\mid-0.1536$ & 0.2068 & 0.6604 & $0.073^{*}$ & \\
\hline $\mathrm{C} 11$ & $0.0570(4)$ & $0.3515(2)$ & 0.1019 (3) & $0.0616(10)$ & \\
\hline H11A & 0.0491 & 0.4052 & 0.1249 & $0.092 *$ & \\
\hline H11B & -0.0148 & 0.3222 & 0.1247 & $0.092 *$ & \\
\hline $\mathrm{H} 11 \mathrm{C}$ & 0.0566 & 0.3481 & 0.0149 & $0.092 *$ & \\
\hline C35 & $0.6748(4)$ & $0.6470(2)$ & $0.1552(3)$ & $0.0578(9)$ & \\
\hline H35A & 0.6020 & 0.6735 & 0.1075 & $0.069^{*}$ & \\
\hline H35B & 0.7496 & 0.6816 & 0.1619 & $0.069 *$ & \\
\hline $\mathrm{C} 28$ & $0.8029(4)$ & $0.4636(2)$ & $0.7860(3)$ & $0.0552(9)$ & \\
\hline $\mathrm{H} 28 \mathrm{~A}$ & 0.7597 & 0.4611 & 0.8585 & $0.066^{*}$ & \\
\hline
\end{tabular}




\begin{tabular}{|c|c|c|c|c|c|}
\hline $\mathrm{H} 28 \mathrm{~B}$ & 0.8128 & 0.4107 & 0.7572 & $0.066^{*}$ & \\
\hline C36 & $0.7051(4)$ & $0.5720(2)$ & 0.0904 (3) & $0.0580(9)$ & \\
\hline H36A & 0.6270 & 0.5403 & 0.0747 & $0.070 *$ & \\
\hline H36B & 0.7328 & 0.5847 & 0.0123 & $0.070 *$ & \\
\hline $\mathrm{C} 13$ & $0.3092(5)$ & 0.3737 (3) & $-0.0001(3)$ & $0.0646(10)$ & \\
\hline H13A & 0.3110 & 0.3211 & -0.0327 & $0.077 *$ & \\
\hline H13B & 0.2315 & 0.3993 & -0.0406 & $0.077^{*}$ & \\
\hline $\mathrm{C} 21$ & $0.3560(4)$ & $0.5845(2)$ & $0.5866(4)$ & $0.0593(9)$ & \\
\hline $\mathrm{H} 21 \mathrm{~A}$ & 0.4230 & 0.6121 & 0.5580 & $0.071 *$ & \\
\hline $\mathrm{H} 21 \mathrm{~B}$ & 0.3475 & 0.5855 & 0.6695 & $0.071 *$ & \\
\hline C14 & $0.4250(5)$ & 0.4168 (3) & $-0.0289(3)$ & $0.0713(11)$ & \\
\hline H14 & 0.5053 & 0.4042 & 0.0154 & $0.086 *$ & \\
\hline C27A & $0.5001(5)$ & 0.3354 (3) & $0.6809(5)$ & $0.0544(16)$ & $0.755(13)$ \\
\hline $\mathrm{H} 27 \mathrm{~A}$ & 0.4325 & 0.3675 & 0.6463 & $0.065^{*}$ & $0.755(13)$ \\
\hline $\mathrm{H} 27 \mathrm{~B}$ & 0.5202 & 0.3319 & 0.7656 & $0.065^{*}$ & $0.755(13)$ \\
\hline $\mathrm{C} 26 \mathrm{~A}$ & $0.5642(14)$ & $0.2968(9)$ & $0.6133(8)$ & $0.076(3)$ & $0.755(13)$ \\
\hline $\mathrm{H} 26 \mathrm{~A}$ & 0.6311 & 0.2653 & 0.6512 & $0.091 *$ & $0.755(13)$ \\
\hline $\mathrm{C} 15$ & $0.4222(6)$ & 0.4713 (4) & $-0.1123(4)$ & $0.0968(18)$ & \\
\hline H15A & 0.3436 & 0.4853 & -0.1582 & $0.116^{*}$ & \\
\hline H15B & 0.4989 & 0.4960 & -0.1257 & $0.116^{*}$ & \\
\hline $\mathrm{Cl} 2 \mathrm{~B}$ & $0.725(2)$ & $0.2912(18)$ & $-0.040(2)$ & $0.067(2)$ & $0.292(7)$ \\
\hline $\mathrm{Cl} 3 \mathrm{~A}$ & 0.6037 (5) & $0.2166(5)$ & $0.1336(6)$ & $0.095(2)$ & $0.395(7)$ \\
\hline
\end{tabular}




\begin{tabular}{|c|c|c|c|c|c|}
\hline Cl1B & $0.6463(7)$ & 0.2599 (4) & $0.1795(5)$ & $0.078(2)$ & $0.292(7)$ \\
\hline $\mathrm{Cl} 3 \mathrm{~B}$ & $0.6956(7)$ & 0.1241 (3) & $0.0456(5)$ & $0.0750(19)$ & $0.292(7)$ \\
\hline $\mathrm{Cl} 2 \mathrm{~A}$ & $0.7838(9)$ & 0.1355 (3) & $0.0000(6)$ & $0.137(3)$ & $0.395(7)$ \\
\hline C1SB & $0.7446(6)$ & 0.2217 (3) & $0.0764(5)$ & $0.0551(12)$ & $0.292(7)$ \\
\hline H1SB & 0.8355 & 0.2221 & 0.1151 & $0.066^{*}$ & $0.292(7)$ \\
\hline C1SA & $0.7446(6)$ & 0.2217 (3) & $0.0764(5)$ & $0.0551(12)$ & $0.395(7)$ \\
\hline H1SA & 0.8154 & 0.2347 & 0.1416 & $0.066^{*}$ & $0.395(7)$ \\
\hline Cl1A & 0.7488 (14) & $0.2853(13)$ & $-0.0501(16)$ & 0.067 (2) & $0.395(7)$ \\
\hline $\mathrm{Cl} 3 \mathrm{C}$ & $0.7739(9)$ & $0.1852(6)$ & $0.0273(8)$ & $0.0551(12)$ & $0.110(2)$ \\
\hline $\mathrm{Cl1C}$ & $0.5442(10)$ & $0.1970(5)$ & $0.1415(10)$ & $0.052(2)$ & $0.110(2)$ \\
\hline $\mathrm{Cl} 2 \mathrm{C}$ & $0.7120(9)$ & $0.3240(5)$ & $0.1564(12)$ & 0.077 (3) & $0.110(2)$ \\
\hline $\mathrm{C} 1 \mathrm{SC}$ & $0.702(2)$ & $0.2230(14)$ & $0.150(3)$ & $0.0551(12)$ & $0.110(2)$ \\
\hline H1SC & 0.7489 & 0.2019 & 0.2260 & $0.066^{*}$ & $0.110(2)$ \\
\hline C27B & $0.5741(17)$ & $0.2716(12)$ & $0.7310(14)$ & $0.072(6)$ & $0.245(13)$ \\
\hline $\mathrm{H} 27 \mathrm{C}$ & 0.6005 & 0.2197 & 0.7405 & $0.087^{*}$ & $0.245(13)$ \\
\hline H27D & 0.5646 & 0.3017 & 0.7994 & $0.087^{*}$ & $0.245(13)$ \\
\hline $\mathrm{C} 26 \mathrm{~B}$ & 0.549 (4) & $0.304(2)$ & $0.6142(16)$ & $0.070(8)$ & 0.245 (13) \\
\hline H26B & 0.5247 & 0.3555 & 0.6275 & $0.083 *$ & $0.245(13)$ \\
\hline Cl3D & 0.8970 (16) & $0.1341(12)$ & $-0.0529(16)$ & $0.095(2)$ & 0.077 (3) \\
\hline $\mathrm{Cl} 2 \mathrm{D}$ & 0.7449 (19) & $0.2470(10)$ & $-0.0543(15)$ & $0.0750(19)$ & 0.077 (3) \\
\hline C1SD & 0.802 (3) & 0.1674 (19) & $0.046(3)$ & $0.0551(12)$ & 0.077 (3) \\
\hline H1SD & 0.8479 & 0.1831 & 0.1264 & $0.066^{*}$ & 0.077 (3) \\
\hline
\end{tabular}




\begin{tabular}{|l|l|l|l|l|l|}
\hline Cl1D & $0.6871(18)$ & $0.0974(11)$ & $0.051(2)$ & $0.077(3)$ & $0.077(3)$ \\
\hline
\end{tabular}

\section{B. Compound 21}

\section{$8^{\mathrm{C}}$}

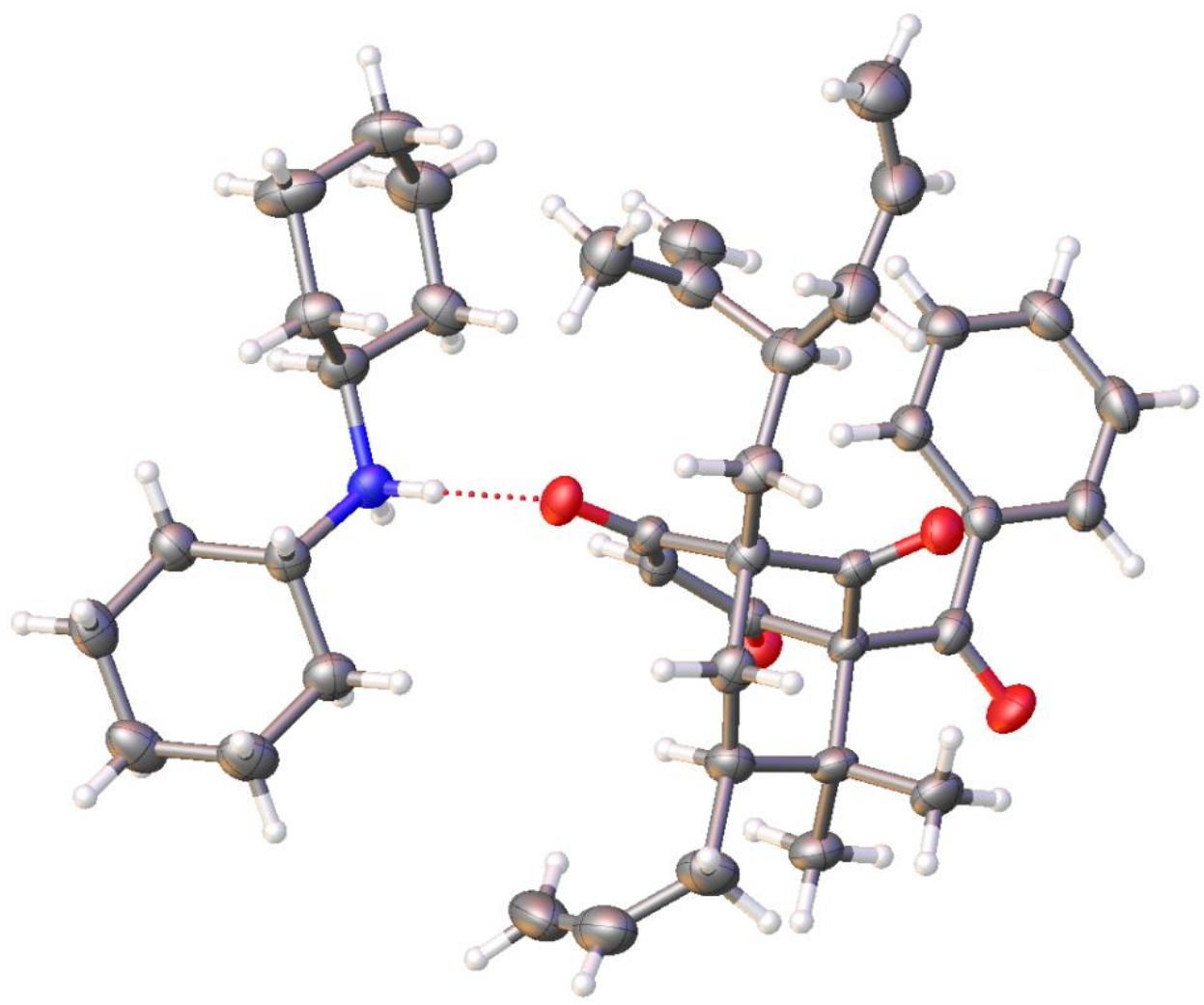

$\stackrel{9}{9}$

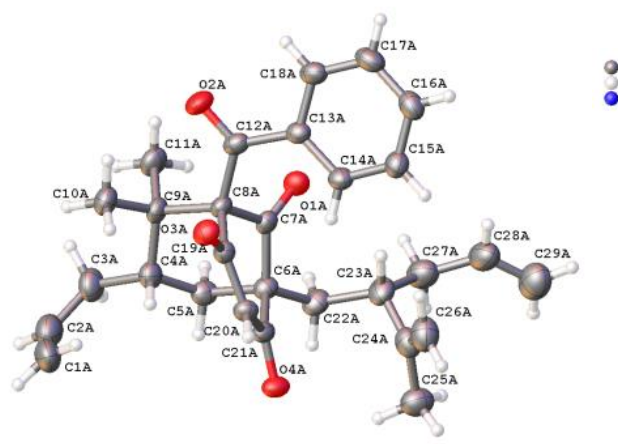

$\stackrel{9}{\rightarrow=}$

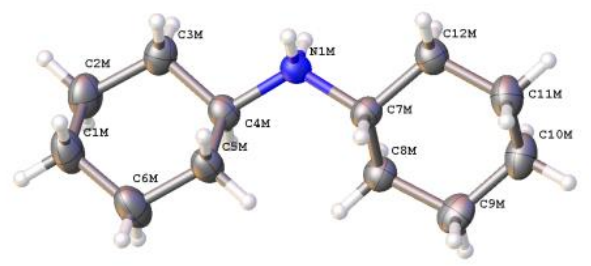



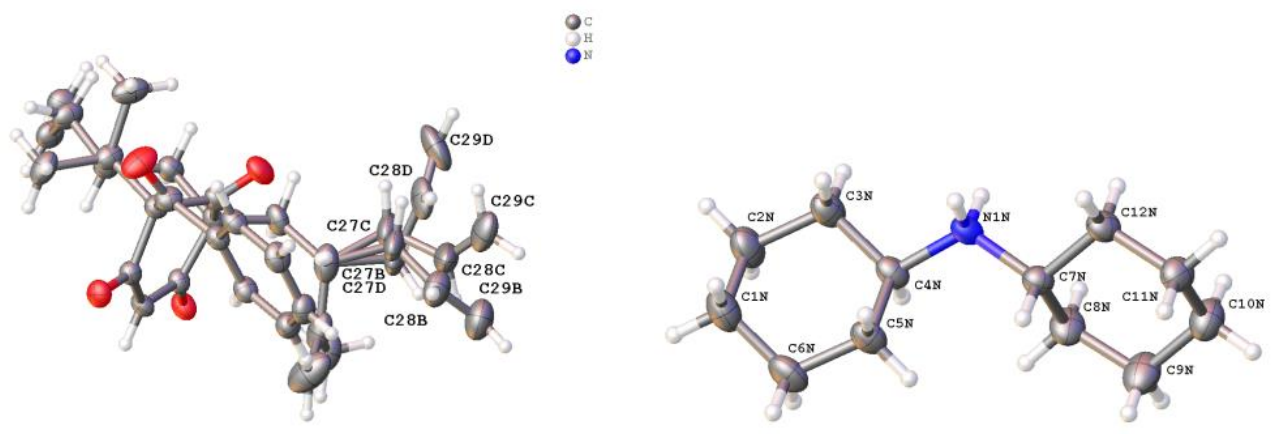

Crystals of compound 21 suitable for X-ray analysis were obtained by layering a $\mathrm{CHCl}_{3}$ solution with isooctane at ambient temperature followed by storage in a sealed vial. Crystallographic data have been deposited with the Cambridge Crystallographic Data Centre (CCDC \# 1913294). Copies of the data can be obtained free of charge through application to the CCDC, 12 Union Road, Cambridge CB21EZ, UK (fax: (+44)-1223-336-033; e-mail: deposit@ccdc.cam.ac.uk).

\section{Computing details}

Data collection: APEX2 (Bruker, 2016); cell refinement: SAINT V8.38A (Bruker, 2016); data reduction: SAINT V8.38A (Bruker, 2016); program(s) used to solve structure: SHELXT 2018/2 (Sheldrick, 2018); program(s) used to refine structure: SHELXL (Sheldrick, 2015); molecular graphics: Olex2 (Dolomanov et al., 2009); software used to prepare material for publication: Olex2 (Dolomanov et al., 2009).

\section{Table S6. Crystal data.}

\begin{tabular}{|l|l|}
\hline $1\left(\mathrm{C}_{29} \mathrm{H}_{33} \mathrm{O}_{4}\right) \cdot \mathrm{C}_{12} \mathrm{H}_{24} \mathrm{~N}$ & $F(000)=2736$ \\
\hline$M_{r}=627.87$ & $D_{\mathrm{x}}=1.142 \mathrm{Mg} \mathrm{m}^{-3}$ \\
\hline Monoclinic, $C 2$ & $\mathrm{Cu} K \alpha$ radiation, $\lambda=1.54178 \AA$ \\
\hline$a=33.1205(10) \AA$ & Cell parameters from 9835 reflections \\
\hline$b=11.3921(3) \AA$ & $\theta=2.3-66.3^{\circ}$ \\
\hline
\end{tabular}




\begin{tabular}{|l|l|}
\hline$c=25.2617(13) \AA$ & $\mu=0.56 \mathrm{~mm}^{-1}$ \\
\hline$\beta=129.951(2)^{\circ}$ & $T=100 \mathrm{~K}$ \\
\hline$V=7306.8(5) \AA^{3}$ & Plate \\
\hline$Z=8$ & $0.12 \times 0.10 \times 0.04 \mathrm{~mm}$ \\
\hline
\end{tabular}

Table S7. Data collection.

\begin{tabular}{|l|l|}
\hline Bruker X8 Proteum-R & 12802 independent reflections \\
diffractometer & 11261 reflections with $I>2 \sigma(I)$ \\
\hline Radiation source: rotating anode & $R_{\mathrm{int}}=0.056$ \\
\hline $\begin{array}{l}\text { Montel monochromator } \\
\text { and } \omega \text { scans }\end{array}$ & $\theta_{\max }=66.8^{\circ}, \theta_{\min }=2.3^{\circ}$ \\
\hline $\begin{array}{l}\text { Absorption correction: multi-scan } \\
\text { absorption correction. } w \mathrm{R} 2 \text { (int) was } 0.0803\end{array}$ & $h=-39 \varnothing 39$ \\
before and 0.0660 after correction. The Ratio of & \\
minimum to maximum transmission is 0.8755. & \\
The $\lambda / 2$ correction factor is Not present. & \\
\hline$T_{\text {min }}=0.659, T_{\max }=0.753$ & $k=-13 \varnothing 13$ \\
\hline 89574 measured reflections & $l=-29 \varnothing 29$ \\
\hline
\end{tabular}

Table S8. Refinement parameters.

\begin{tabular}{|l|l|}
\hline Refinement on $F^{2}$ & $\begin{array}{l}\text { Hydrogen site location: inferred from } \\
\text { neighbouring sites }\end{array}$ \\
\hline Least-squares matrix: full & H-atom parameters constrained \\
\hline
\end{tabular}




\begin{tabular}{|l|l|}
\hline$R\left[F^{2}>2 \sigma\left(F^{2}\right)\right]=0.041$ & $\begin{array}{l}w=1 /\left[\sigma^{2}\left(F_{\mathrm{o}}{ }^{2}\right)+(0.0634 P)^{2}+2.1555 P\right] \\
\text { where } P=\left(F_{\mathrm{o}}{ }^{2}+2 F_{\mathrm{c}}{ }^{2}\right) / 3\end{array}$ \\
\hline$w R\left(F^{2}\right)=0.110$ & $(\Delta / \sigma)_{\max }=0.001$ \\
\hline$S=1.06$ & $\Delta\rangle_{\max }=0.25 \mathrm{e} \AA^{-3}$ \\
\hline 12802 reflections & $\Delta\rangle_{\min }=-0.24 \mathrm{e} \AA^{-3}$ \\
\hline 880 parameters & Absolute structure: Flack x determined using \\
& 4686 quotients [(I+)-(I-)]/[(I+)+(I-)] (Parsons, \\
& Flack and Wagner, Acta Cryst. B69 (2013) 249- \\
& $259)$. \\
\hline 27 restraints & Absolute structure parameter: -0.02 (6) \\
\hline
\end{tabular}

\section{Special details}

Geometry. All esds (except the esd in the dihedral angle between two 1.s. planes) are estimated using the full covariance matrix. The cell esds are taken into account individually in the estimation of esds in distances, angles and torsion angles; correlations between esds in cell parameters are only used when they are defined by crystal symmetry. An approximate (isotropic) treatment of cell esds is used for estimating esds involving l.s. planes.

Table S9. Fractional atomic coordinates and isotropic or equivalent isotropic displacement parameters $\left(\AA^{2}\right)$ for 21.

\begin{tabular}{|l|l|l|l|l|l|}
\hline & $x$ & $y$ & $z$ & $U_{\text {iso }} * / U_{\text {eq }}$ & Occ. $(<1)$ \\
\hline O3B & $0.58378(8)$ & $0.72429(18)$ & $1.10783(10)$ & $0.0316(4)$ & \\
\hline O4B & $0.54578(7)$ & $0.60028(19)$ & $0.90265(10)$ & $0.0319(4)$ & \\
\hline O1A & $0.87535(8)$ & $0.97255(19)$ & $1.59035(11)$ & $0.0348(5)$ & \\
\hline O3A & $1.01190(7)$ & $0.87127(19)$ & $1.59905(10)$ & $0.0350(5)$ & \\
\hline
\end{tabular}




\begin{tabular}{|c|c|c|c|c|}
\hline $\mathrm{O} 4 \mathrm{~A}$ & $0.84063(8)$ & $0.89348(19)$ & $1.38435(10)$ & $0.0347(5)$ \\
\hline O1B & $0.71402(7)$ & $0.7183(2)$ & $1.09701(11)$ & $0.0383(5)$ \\
\hline $\mathrm{O} 2 \mathrm{~A}$ & $1.00607(9)$ & $0.8640(2)$ & $1.72490(11)$ & $0.0447(6)$ \\
\hline $\mathrm{O} 2 \mathrm{~B}$ & $0.69361(10)$ & $0.8749(2)$ & $1.20209(11)$ & $0.0485(6)$ \\
\hline N1N & $0.56069(9)$ & $0.5808(2)$ & $1.17251(12)$ & $0.0277(5)$ \\
\hline H1NA & 0.525984 & 0.582742 & 1.148134 & $0.033^{*}$ \\
\hline $\mathrm{H} 1 \mathrm{NB}$ & 0.567693 & 0.623647 & 1.149809 & $0.033^{*}$ \\
\hline N1M & $0.87884(9)$ & $0.8802(2)$ & $1.31406(12)$ & 0.0309 (5) \\
\hline H1MA & 0.867865 & 0.884960 & 1.338209 & $0.037 *$ \\
\hline H1MB & 0.913664 & 0.871466 & 1.344223 & $0.037 *$ \\
\hline $\mathrm{C} 21 \mathrm{~B}$ & $0.57574(10)$ & $0.6444(2)$ & 0.96207 (14) & $0.0264(6)$ \\
\hline C7B & $0.66650(10)$ & $0.7262(3)$ & $1.06181(14)$ & $0.0284(6)$ \\
\hline C19B & $0.59316(10)$ & $0.7168(3)$ & $1.06689(14)$ & $0.0267(6)$ \\
\hline $\mathrm{C} 20 \mathrm{~B}$ & $0.56338(10)$ & $0.6536(3)$ & $1.00560(14)$ & $0.0268(6)$ \\
\hline H20B & 0.533479 & 0.615023 & 0.992661 & $0.032 *$ \\
\hline C7A & $0.88700(11)$ & $0.9168(2)$ & $1.56077(15)$ & $0.0286(6)$ \\
\hline $\mathrm{C} 20 \mathrm{~A}$ & 0.92727 (11) & 0.8927 (3) & $1.49042(14)$ & $0.0305(6)$ \\
\hline $\mathrm{H} 20 \mathrm{~A}$ & 0.941302 & 0.904739 & 1.468654 & $0.037 *$ \\
\hline C13A & $0.98840(11)$ & $1.0500(3)$ & $1.67422(15)$ & $0.0304(6)$ \\
\hline $\mathrm{C} 18 \mathrm{~B}$ & $0.74783(11)$ & 0.6785 (3) & $1.27784(15)$ & $0.0321(6)$ \\
\hline H18B & 0.760868 & 0.751242 & 1.299154 & $0.038 *$ \\
\hline C13B & $0.70568(10)$ & $0.6720(3)$ & $1.20680(14)$ & $0.0288(6)$ \\
\hline
\end{tabular}




\begin{tabular}{|c|c|c|c|c|}
\hline C14A & $0.96886(12)$ & $1.1197(3)$ & $1.61666(16)$ & 0.0339 (7) \\
\hline H14A & 0.949410 & 1.085511 & 1.573153 & $0.041 *$ \\
\hline $\mathrm{C} 2 \mathrm{~B}$ & $0.53003(13)$ & $1.0096(3)$ & $0.86072(16)$ & $0.0378(7)$ \\
\hline $\mathrm{H} 2 \mathrm{~B}$ & 0.498405 & 0.982134 & 0.847953 & $0.045^{*}$ \\
\hline $\mathrm{C} 6 \mathrm{~B}$ & $0.62764(10)$ & 0.6959 (3) & 0.98541 (14) & $0.0276(6)$ \\
\hline $\mathrm{C} 4 \mathrm{~N}$ & $0.58618(11)$ & $0.6395(3)$ & $1.24070(14)$ & $0.0304(6)$ \\
\hline $\mathrm{H} 4 \mathrm{~N}$ & 0.573636 & 0.601469 & 1.262570 & $0.037 *$ \\
\hline $\mathrm{C} 12 \mathrm{~N}$ & $0.54973(12)$ & $0.4196(3)$ & $1.09964(15)$ & $0.0337(7)$ \\
\hline $\mathrm{H} 12 \mathrm{~A}$ & 0.511797 & 0.426463 & 1.071117 & $0.040 *$ \\
\hline H12B & 0.561032 & 0.471636 & 1.080952 & $0.040 *$ \\
\hline $\mathrm{C} 7 \mathrm{~N}$ & $0.57592(11)$ & $0.4565(3)$ & $1.17360(15)$ & $0.0293(6)$ \\
\hline $\mathrm{H} 7 \mathrm{~N}$ & 0.614196 & 0.453268 & 1.201074 & $0.035^{*}$ \\
\hline C19A & $0.96231(11)$ & $0.8756(2)$ & $1.56189(14)$ & $0.0286(6)$ \\
\hline C9B & $0.62468(12)$ & $0.9136(3)$ & $1.05441(15)$ & $0.0338(7)$ \\
\hline C14B & $0.68770(11)$ & 0.5614 (3) & $1.17638(15)$ & $0.0311(6)$ \\
\hline H14B & 0.660262 & 0.555389 & 1.129040 & $0.037 *$ \\
\hline $\mathrm{C} 21 \mathrm{~A}$ & $0.87238(11)$ & $0.8929(2)$ & $1.44953(14)$ & $0.0289(6)$ \\
\hline $\mathrm{C} 8 \mathrm{~A}$ & $0.94046(11)$ & $0.8596(2)$ & $1.59973(14)$ & $0.0273(6)$ \\
\hline C15A & $0.97836(12)$ & $1.2393(3)$ & $1.62420(17)$ & $0.0378(7)$ \\
\hline H15A & 0.965443 & 1.285121 & 1.585760 & $0.045^{*}$ \\
\hline C22B & $0.65269(12)$ & $0.6199(3)$ & $0.96360(16)$ & $0.0364(7)$ \\
\hline $\mathrm{H} 22 \mathrm{~A}$ & 0.681455 & 0.663726 & 0.972085 & $0.044 *$ \\
\hline
\end{tabular}




\begin{tabular}{|c|c|c|c|c|}
\hline $\mathrm{H} 22 \mathrm{~B}$ & 0.626690 & 0.606721 & 0.914210 & $0.044 *$ \\
\hline C4B & $0.58936(11)$ & $0.9005(3)$ & $0.97385(15)$ & $0.0323(6)$ \\
\hline H4B & 0.556112 & 0.864555 & 0.957075 & $0.039 *$ \\
\hline $\mathrm{C} 5 \mathrm{~N}$ & $0.64595(11)$ & $0.6292(3)$ & $1.28887(15)$ & $0.0361(7)$ \\
\hline H5NA & 0.658476 & 0.657655 & 1.265474 & $0.043 *$ \\
\hline H5NB & 0.655983 & 0.547365 & 1.300540 & $0.043 *$ \\
\hline $\mathrm{C} 12 \mathrm{~B}$ & $0.68175(11)$ & $0.7858(3)$ & $1.16882(15)$ & $0.0322(6)$ \\
\hline $\mathrm{C} 8 \mathrm{~B}$ & $0.64185(10)$ & $0.7857(3)$ & 1.08855 (14) & $0.0273(6)$ \\
\hline C6A & 0.84765 (11) & $0.8855(3)$ & 1.48464 (14) & $0.0303(6)$ \\
\hline C17B & 0.77014 (12) & 0.5767 (3) & $1.31642(16)$ & $0.0371(7)$ \\
\hline H17B & 0.798368 & 0.581758 & 1.363538 & $0.045^{*}$ \\
\hline $\mathrm{C} 11 \mathrm{~N}$ & $0.56406(13)$ & 0.2937 (3) & 1.09767 (17) & $0.0374(7)$ \\
\hline $\mathrm{H} 11 \mathrm{G}$ & 0.601674 & 0.288120 & 1.123111 & $0.045^{*}$ \\
\hline $\mathrm{H} 11 \mathrm{H}$ & 0.546015 & 0.270906 & 1.050239 & $0.045^{*}$ \\
\hline $\mathrm{C} 7 \mathrm{M}$ & $0.85574(12)$ & $0.7715(3)$ & $1.27094(16)$ & 0.0337 (7) \\
\hline $\mathrm{H} 7 \mathrm{M}$ & 0.817421 & 0.773658 & 1.243236 & $0.040^{*}$ \\
\hline $\mathrm{C} 4 \mathrm{M}$ & $0.86712(12)$ & 0.9948 (3) & $1.27786(17)$ & $0.0358(7)$ \\
\hline $\mathrm{H} 4 \mathrm{M}$ & 0.882976 & 0.993876 & 1.255844 & $0.043 *$ \\
\hline C5B & $0.61341(11)$ & $0.8201(3)$ & 0.95235 (16) & $0.0332(7)$ \\
\hline H5BA & 0.645034 & 0.856732 & 0.965152 & $0.040 *$ \\
\hline H5BB & 0.588714 & 0.811962 & 0.902491 & $0.040^{*}$ \\
\hline C16B & $0.75113(12)$ & $0.4682(3)$ & $1.28601(17)$ & $0.0376(7)$ \\
\hline
\end{tabular}




\begin{tabular}{|c|c|c|c|c|}
\hline H16B & 0.765793 & 0.400395 & 1.312653 & $0.045^{*}$ \\
\hline $\mathrm{C} 12 \mathrm{~A}$ & 0.98054 (11) & $0.9195(3)$ & $1.67094(15)$ & $0.0328(7)$ \\
\hline C18A & $1.01702(12)$ & $1.1032(3)$ & $1.73859(16)$ & $0.0377(7)$ \\
\hline $\mathrm{H} 18 \mathrm{~A}$ & 1.030361 & 1.058080 & 1.777404 & $0.045^{*}$ \\
\hline $\mathrm{C} 8 \mathrm{M}$ & 0.86809 (13) & $0.7618(3)$ & $1.22253(17)$ & $0.0382(7)$ \\
\hline H8MA & 0.905828 & 0.767875 & 1.248958 & $0.046^{*}$ \\
\hline H8MB & 0.851365 & 0.825936 & 1.189608 & $0.046^{*}$ \\
\hline $\mathrm{C} 12 \mathrm{M}$ & 0.87759 (13) & $0.6673(3)$ & 1.32019 (18) & $0.0412(7)$ \\
\hline $\mathrm{H} 12 \mathrm{C}$ & 0.915764 & 0.668859 & 1.351136 & $0.049^{*}$ \\
\hline H12D & 0.866829 & 0.673171 & 1.347887 & $0.049 *$ \\
\hline $\mathrm{C} 1 \mathrm{~B}$ & 0.53067 (14) & $1.0363(3)$ & 0.81108 (19) & 0.0492 (9) \\
\hline H1BA & 0.561450 & 1.064034 & 0.821464 & $0.059^{*}$ \\
\hline H1BB & 0.500335 & 1.027565 & 0.765280 & $0.059 *$ \\
\hline C9A & $0.92991(12)$ & $0.7235(3)$ & $1.60328(16)$ & $0.0342(7)$ \\
\hline $\mathrm{C} 3 \mathrm{~N}$ & $0.56837(12)$ & $0.7662(3)$ & $1.22605(16)$ & $0.0373(7)$ \\
\hline H3NA & 0.530479 & 0.769130 & 1.198887 & $0.045^{*}$ \\
\hline $\mathrm{H} 3 \mathrm{NB}$ & 0.576615 & 0.801878 & 1.199133 & $0.045^{*}$ \\
\hline C15B & $0.71017(12)$ & $0.4604(3)$ & $1.21574(16)$ & $0.0349(7)$ \\
\hline H15B & 0.697656 & 0.387224 & 1.194826 & $0.042 *$ \\
\hline $\mathrm{C} 2 \mathrm{~N}$ & $0.59506(13)$ & $0.8362(3)$ & $1.29282(18)$ & $0.0467(8)$ \\
\hline $\mathrm{H} 2 \mathrm{NA}$ & 0.584541 & 0.917866 & 1.281562 & $0.056^{*}$ \\
\hline $\mathrm{H} 2 \mathrm{NB}$ & 0.583533 & 0.806327 & 1.317169 & $0.056^{*}$ \\
\hline
\end{tabular}




\begin{tabular}{|c|c|c|c|c|}
\hline $\mathrm{C} 5 \mathrm{~A}$ & $0.83682(12)$ & $0.7546(3)$ & $1.48643(16)$ & $0.0348(7)$ \\
\hline H5AA & 0.819937 & 0.747104 & 1.506498 & $0.042 *$ \\
\hline $\mathrm{H} 5 \mathrm{AB}$ & 0.812504 & 0.725249 & 1.439467 & $0.042 *$ \\
\hline C17A & $1.02577(13)$ & $1.2231(3)$ & $1.74523(18)$ & $0.0453(8)$ \\
\hline H17A & 1.044563 & 1.258127 & 1.788418 & $0.054 *$ \\
\hline $\mathrm{C} 10 \mathrm{~B}$ & $0.59221(14)$ & $0.9739(3)$ & $1.07041(18)$ & $0.0438(8)$ \\
\hline H10A & 0.585376 & 1.053776 & 1.054606 & $0.066^{*}$ \\
\hline H10B & 0.611488 & 0.972723 & 1.119325 & $0.066^{*}$ \\
\hline $\mathrm{H} 10 \mathrm{C}$ & 0.559430 & 0.933141 & 1.047304 & $0.066^{*}$ \\
\hline $\mathrm{C} 3 \mathrm{~B}$ & 0.57569 (13) & $1.0191(3)$ & $0.93651(16)$ & 0.0408 (7) \\
\hline H3BA & 0.567296 & 1.075551 & 0.956908 & $0.049 *$ \\
\hline H3BB & 0.606184 & 1.047921 & 0.943051 & $0.049^{*}$ \\
\hline $\mathrm{C} 8 \mathrm{~N}$ & 0.56098 (14) & $0.3742(3)$ & 1.20575 (17) & $0.0403(7)$ \\
\hline H8NA & 0.523514 & 0.380361 & 1.181176 & $0.048 *$ \\
\hline H8NB & 0.579719 & 0.396387 & 1.253453 & $0.048 *$ \\
\hline $\mathrm{C} 10 \mathrm{~N}$ & $0.54849(15)$ & $0.2113(3)$ & 1.12931 (19) & $0.0454(8)$ \\
\hline H10G & 0.510535 & 0.212313 & 1.101677 & $0.055^{*}$ \\
\hline $\mathrm{H} 10 \mathrm{H}$ & 0.559016 & 0.131809 & 1.129368 & $0.055^{*}$ \\
\hline $\mathrm{C} 3 \mathrm{M}$ & $0.89298(13)$ & 1.0915 (3) & $1.33147(19)$ & $0.0424(8)$ \\
\hline H3MA & 0.930764 & 1.079061 & 1.364071 & $0.051^{*}$ \\
\hline H3MB & 0.880100 & 1.089149 & 1.356759 & $0.051^{*}$ \\
\hline C5M & $0.80826(12)$ & 1.0139 (3) & $1.22225(17)$ & $0.0415(8)$ \\
\hline
\end{tabular}




\begin{tabular}{|c|c|c|c|c|c|}
\hline H5MA & 0.791495 & 1.011061 & 1.242617 & $0.050 *$ & \\
\hline H5MB & 0.793360 & 0.952021 & 1.188007 & $0.050 *$ & \\
\hline $\mathrm{C} 9 \mathrm{~N}$ & $0.57460(18)$ & $0.2482(3)$ & $1.2027(2)$ & 0.0517 (9) & \\
\hline H9NA & 0.612495 & 0.240721 & 1.231062 & $0.062 *$ & \\
\hline H9NB & 0.563266 & 0.196276 & 1.221364 & $0.062 *$ & \\
\hline $\mathrm{C} 23 \mathrm{~B}$ & $0.67402(14)$ & 0.4999 (3) & 0.99948 (18) & $0.0484(9)$ & \\
\hline H23B & 0.691252 & 0.513770 & 1.048133 & $0.058^{*}$ & $0.433(11)$ \\
\hline $\mathrm{H} 23 \mathrm{~A}$ & 0.692991 & 0.512430 & 1.048761 & $0.058 *$ & $0.407(11)$ \\
\hline $\mathrm{H} 23 \mathrm{C}$ & 0.694758 & 0.510989 & 1.049274 & $0.058 *$ & $0.160(8)$ \\
\hline $\mathrm{C} 23 \mathrm{~A}$ & $0.79941(12)$ & $1.0883(3)$ & 1.44784 (17) & $0.0428(8)$ & \\
\hline $\mathrm{H} 23 \mathrm{D}$ & 0.832941 & 1.110371 & 1.492515 & $0.051^{*}$ & \\
\hline $\mathrm{C} 6 \mathrm{~N}$ & $0.67173(12)$ & $0.7000(3)$ & $1.35501(17)$ & $0.0430(8)$ & \\
\hline H6NA & 0.662311 & 0.665834 & 1.380957 & $0.052 *$ & \\
\hline H6NB & 0.709721 & 0.695820 & 1.383383 & $0.052 *$ & \\
\hline $\mathrm{C} 24 \mathrm{~A}$ & $0.79936(13)$ & $1.1379(3)$ & 1.39347 (19) & $0.0442(8)$ & \\
\hline $\mathrm{C} 4 \mathrm{~A}$ & 0.88648 (13) & $0.6788(3)$ & 1.52765 (17) & $0.0382(7)$ & \\
\hline $\mathrm{H} 4 \mathrm{~A}$ & 0.901305 & 0.684430 & 1.504405 & $0.046^{*}$ & \\
\hline C16A & $1.00686(13)$ & 1.2909 (3) & $1.68837(18)$ & $0.0428(8)$ & \\
\hline H16A & 1.013294 & 1.371291 & 1.693260 & $0.051 *$ & \\
\hline $\mathrm{C} 9 \mathrm{M}$ & $0.84848(14)$ & $0.6452(3)$ & $1.18396(19)$ & $0.0458(8)$ & \\
\hline H9MA & 0.810339 & 0.642749 & 1.153825 & $0.055^{*}$ & \\
\hline H9MB & 0.858502 & 0.638695 & 1.155462 & $0.055^{*}$ & \\
\hline
\end{tabular}




\begin{tabular}{|c|c|c|c|c|}
\hline $\mathrm{C} 1 \mathrm{~N}$ & $0.65448(13)$ & 0.8277 (3) & $1.33921(18)$ & $0.0469(8)$ \\
\hline $\mathrm{H} 1 \mathrm{NC}$ & 0.670328 & 0.869562 & 1.381943 & $0.056^{*}$ \\
\hline H1ND & 0.666405 & 0.863902 & 1.316639 & $0.056^{*}$ \\
\hline $\mathrm{C} 22 \mathrm{~A}$ & $0.79583(11)$ & $0.9533(3)$ & $1.44650(17)$ & $0.0375(7)$ \\
\hline $\mathrm{H} 22 \mathrm{C}$ & 0.779605 & 0.930883 & 1.466105 & $0.045^{*}$ \\
\hline $\mathrm{H} 22 \mathrm{D}$ & 0.772433 & 0.928099 & 1.398647 & $0.045^{*}$ \\
\hline $\mathrm{C} 24 \mathrm{~B}$ & $0.63366(16)$ & $0.4065(3)$ & $0.9754(2)$ & $0.0513(9)$ \\
\hline C11A & $0.91225(14)$ & $0.7094(3)$ & $1.64586(18)$ & $0.0429(8)$ \\
\hline H11D & 0.940325 & 0.732599 & 1.692702 & $0.064 *$ \\
\hline H11E & 0.903336 & 0.628792 & 1.644901 & $0.064 *$ \\
\hline $\mathrm{H} 11 \mathrm{~F}$ & 0.881997 & 0.757921 & 1.626747 & $0.064 *$ \\
\hline $\mathrm{C} 11 \mathrm{~B}$ & $0.67312(14)$ & 0.9877 (3) & $1.08210(17)$ & $0.0475(8)$ \\
\hline H11A & 0.693617 & 0.948804 & 1.072670 & $0.071^{*}$ \\
\hline H11B & 0.694060 & 0.997733 & 1.131044 & $0.071^{*}$ \\
\hline H11C & 0.662103 & 1.063096 & 1.059973 & $0.071 *$ \\
\hline $\mathrm{C} 2 \mathrm{M}$ & $0.88128(14)$ & $1.2110(3)$ & $1.2977(2)$ & $0.0531(9)$ \\
\hline $\mathrm{H} 2 \mathrm{MA}$ & 0.898801 & 1.217292 & 1.278482 & $0.064^{*}$ \\
\hline $\mathrm{H} 2 \mathrm{MB}$ & 0.895387 & 1.271611 & 1.332550 & $0.064 *$ \\
\hline C10A & $0.98024(13)$ & 0.6519 (3) & $1.63639(18)$ & $0.0426(8)$ \\
\hline H10D & 0.992851 & 0.665635 & 1.611719 & $0.064^{*}$ \\
\hline H10E & 0.972715 & 0.569949 & 1.634511 & $0.064 *$ \\
\hline $\mathrm{H} 10 \mathrm{~F}$ & 1.006654 & 0.675460 & 1.683664 & $0.064 *$ \\
\hline
\end{tabular}




\begin{tabular}{|c|c|c|c|c|c|}
\hline $\mathrm{C} 10 \mathrm{M}$ & $0.87133(15)$ & $0.5421(3)$ & $1.2340(2)$ & $0.0498(9)$ & \\
\hline H10I & 0.857361 & 0.469201 & 1.208187 & $0.060 *$ & \\
\hline H10J & 0.909304 & 0.540814 & 1.261727 & $0.060 *$ & \\
\hline $\mathrm{C} 1 \mathrm{M}$ & $0.82230(15)$ & $1.2314(3)$ & $1.2406(2)$ & $0.0554(10)$ & \\
\hline $\mathrm{H} 1 \mathrm{MC}$ & 0.805272 & 1.235739 & 1.260534 & $0.066^{*}$ & \\
\hline H1MD & 0.816733 & 1.305502 & 1.217829 & $0.066^{*}$ & \\
\hline $\mathrm{C} 25 \mathrm{~B}$ & $0.60136(16)$ & $0.3641(4)$ & $0.90246(19)$ & $0.0555(9)$ & \\
\hline $\mathrm{H} 25 \mathrm{~A}$ & 0.582565 & 0.428935 & 0.871403 & $0.083 *$ & \\
\hline $\mathrm{H} 25 \mathrm{~B}$ & 0.576722 & 0.306081 & 0.893818 & $0.083^{*}$ & \\
\hline $\mathrm{H} 25 \mathrm{C}$ & 0.624098 & 0.330056 & 0.895403 & $0.083 *$ & \\
\hline $\mathrm{C} 11 \mathrm{M}$ & $0.85779(15)$ & $0.5519(3)$ & $1.2804(2)$ & $0.0495(9)$ & \\
\hline H11I & 0.873514 & 0.486920 & 1.312754 & $0.059^{*}$ & \\
\hline H11J & 0.819892 & 0.547167 & 1.252845 & $0.059^{*}$ & \\
\hline C6M & $0.79786(15)$ & $1.1325(3)$ & $1.1878(2)$ & $0.0569(10)$ & \\
\hline H6MA & 0.812506 & 1.133066 & 1.164661 & $0.068^{*}$ & \\
\hline H6MB & 0.760176 & 1.145068 & 1.153257 & $0.068 *$ & \\
\hline $\mathrm{C} 26 \mathrm{~A}$ & $0.84075(15)$ & $1.1976(3)$ & $1.4116(2)$ & $0.0543(9)$ & \\
\hline $\mathrm{H} 26 \mathrm{C}$ & 0.869548 & 1.208241 & 1.458130 & $0.065^{*}$ & \\
\hline $\mathrm{H} 26 \mathrm{D}$ & 0.841103 & 1.229020 & 1.377961 & $0.065^{*}$ & \\
\hline C27B & $0.7168(10)$ & $0.4480(15)$ & $0.9991(18)$ & $0.058(3)$ & $0.433(11)$ \\
\hline $\mathrm{H} 27 \mathrm{~A}$ & 0.702274 & 0.433599 & 0.951923 & $0.070 *$ & $0.433(11)$ \\
\hline H27B & 0.745758 & 0.502859 & 1.020005 & $0.070 *$ & $0.433(11)$ \\
\hline
\end{tabular}




\begin{tabular}{|c|c|c|c|c|}
\hline $\mathrm{C} 28 \mathrm{~A}$ & $0.75919(17)$ & $1.2727(4)$ & $1.4529(2)$ & $0.0602(10)$ \\
\hline $\mathrm{H} 28 \mathrm{~A}$ & 0.790754 & 1.301969 & 1.492656 & $0.072 *$ \\
\hline C3A & $0.87098(16)$ & $0.5493(3)$ & $1.5231(2)$ & $0.0526(9)$ \\
\hline H3AA & 0.889222 & 0.520930 & 1.569644 & $0.063 *$ \\
\hline $\mathrm{H} 3 \mathrm{AB}$ & 0.833544 & 0.546412 & 1.499082 & $0.063^{*}$ \\
\hline C27A & $0.75436(15)$ & $1.1445(4)$ & $1.4442(2)$ & $0.0536(9)$ \\
\hline $\mathrm{H} 27 \mathrm{G}$ & 0.720610 & 1.125691 & 1.399880 & $0.064 *$ \\
\hline $\mathrm{H} 27 \mathrm{H}$ & 0.755087 & 1.110177 & 1.479902 & $0.064 *$ \\
\hline $\mathrm{C} 26 \mathrm{~B}$ & $0.6275(2)$ & $0.3584(4)$ & 1.0177 (3) & $0.0803(15)$ \\
\hline $\mathrm{H} 26 \mathrm{~A}$ & 0.603155 & 0.298436 & 1.002082 & $0.096^{*}$ \\
\hline H26B & 0.647575 & 0.384807 & 1.063051 & $0.096^{*}$ \\
\hline $\mathrm{C} 2 \mathrm{~A}$ & $0.88188(18)$ & $0.4677(4)$ & 1.4885 (2) & $0.0629(11)$ \\
\hline $\mathrm{H} 2 \mathrm{~A}$ & 0.864179 & 0.396273 & 1.475090 & $0.075^{*}$ \\
\hline $\mathrm{C} 25 \mathrm{~A}$ & $0.75076(15)$ & $1.1213(4)$ & $1.31900(19)$ & 0.0539 (9) \\
\hline $\mathrm{H} 25 \mathrm{D}$ & 0.745740 & 1.039179 & 1.307845 & $0.081 *$ \\
\hline $\mathrm{H} 25 \mathrm{E}$ & 0.754971 & 1.162474 & 1.289665 & $0.081 *$ \\
\hline $\mathrm{H} 25 \mathrm{~F}$ & 0.720639 & 1.151590 & 1.312107 & $0.081 *$ \\
\hline $\mathrm{C} 29 \mathrm{~A}$ & $0.72250(19)$ & $1.3502(4)$ & $1.4089(2)$ & $0.0688(12)$ \\
\hline $\mathrm{H} 29 \mathrm{G}$ & 0.690344 & 1.324703 & 1.368485 & $0.083^{*}$ \\
\hline $\mathrm{H} 29 \mathrm{H}$ & 0.728891 & 1.429991 & 1.418558 & $0.083^{*}$ \\
\hline $\mathrm{C} 1 \mathrm{~A}$ & $0.9133(2)$ & 0.4819 (4) & $1.4740(2)$ & $0.0713(13)$ \\
\hline H1AA & 0.932211 & 0.551339 & 1.485989 & $0.086^{*}$ \\
\hline
\end{tabular}




\begin{tabular}{|c|c|c|c|c|c|}
\hline $\mathrm{H} 1 \mathrm{AB}$ & 0.916627 & 0.422565 & 1.451698 & $0.086^{*}$ & \\
\hline $\mathrm{C} 28 \mathrm{~B}$ & $0.7362(5)$ & $0.3341(10)$ & $1.0393(6)$ & 0.053 & $0.433(11)$ \\
\hline $\mathrm{H} 28 \mathrm{~B}$ & 0.748945 & 0.333574 & 1.084507 & $0.063 *$ & $0.433(11)$ \\
\hline $\mathrm{C} 29 \mathrm{~B}$ & 0.7354 (4) & $0.2378(9)$ & $1.0119(6)$ & 0.068 (3) & $0.433(11)$ \\
\hline $\mathrm{H} 29 \mathrm{~A}$ & 0.722700 & 0.237554 & 0.966661 & $0.082^{*}$ & $0.433(11)$ \\
\hline $\mathrm{H} 29 \mathrm{~B}$ & 0.747480 & 0.168589 & 1.037307 & $0.082 *$ & $0.433(11)$ \\
\hline C29C & 0.7995 (4) & $0.3647(9)$ & $1.0801(5)$ & $0.061(3)$ & 0.407 (11) \\
\hline $\mathrm{H} 29 \mathrm{C}$ & 0.819187 & 0.433649 & 1.096854 & $0.074 *$ & 0.407 (11) \\
\hline $\mathrm{H} 29 \mathrm{D}$ & 0.815993 & 0.293011 & 1.099933 & $0.074 *$ & $0.407(11)$ \\
\hline $\mathrm{C} 28 \mathrm{C}$ & $0.7499(5)$ & $0.3686(12)$ & $1.0298(8)$ & $0.067(4)$ & $0.407(11)$ \\
\hline $\mathrm{H} 28 \mathrm{C}$ & 0.732286 & 0.297083 & 1.015230 & $0.080 *$ & $0.407(11)$ \\
\hline C28D & $0.7607(9)$ & $0.485(2)$ & $1.0268(12)$ & $0.056(8)$ & $0.160(8)$ \\
\hline $\mathrm{H} 28 \mathrm{D}$ & 0.782925 & 0.477002 & 1.074562 & $0.068 *$ & $0.160(8)$ \\
\hline C29D & $0.7796(10)$ & $0.543(3)$ & $1.0034(15)$ & $0.074(10)$ & $0.160(8)$ \\
\hline $\mathrm{H} 29 \mathrm{E}$ & 0.759442 & 0.553874 & 0.956131 & $0.089^{*}$ & $0.160(8)$ \\
\hline $\mathrm{H} 29 \mathrm{~F}$ & 0.813387 & 0.574017 & 1.033854 & $0.089 *$ & $0.160(8)$ \\
\hline $\mathrm{C} 27 \mathrm{C}$ & $0.7164(11)$ & $0.477(2)$ & $0.9911(18)$ & 0.058 (3) & 0.407 (11) \\
\hline $\mathrm{H} 27 \mathrm{C}$ & 0.698679 & 0.468235 & 0.942390 & $0.070 *$ & 0.407 (11) \\
\hline H27D & 0.739214 & 0.545313 & 1.007828 & $0.070 *$ & $0.407(11)$ \\
\hline C27D & $0.7072(13)$ & $0.427(3)$ & $0.987(3)$ & $0.058(3)$ & $0.160(8)$ \\
\hline $\mathrm{H} 27 \mathrm{E}$ & 0.711306 & 0.346833 & 1.003533 & $0.070 *$ & $0.160(8)$ \\
\hline $\mathrm{H} 27 \mathrm{~F}$ & 0.689627 & 0.424056 & 0.938453 & $0.070 *$ & $0.160(8)$ \\
\hline
\end{tabular}


Table S10. Atomic displacement parameters $\left(\AA^{2}\right)$ for 21

\begin{tabular}{|c|c|c|c|c|c|c|}
\hline & $U^{11}$ & $U^{22}$ & $U^{33}$ & $U^{12}$ & $U^{13}$ & $U^{23}$ \\
\hline O3B & $0.0358(10)$ & 0.0377 (11) & $0.0271(10)$ & $0.0024(9)$ & $0.0228(9)$ & $-0.0014(8)$ \\
\hline O4B & $0.0264(10)$ & 0.0448 (12) & $0.0231(10)$ & $-0.0007(8)$ & $0.0152(9)$ & $-0.0048(9)$ \\
\hline $\mathrm{O} 1 \mathrm{~A}$ & $0.0359(11)$ & $0.0395(11)$ & $0.0332(11)$ & $0.0043(9)$ & $0.0240(10)$ & $0.0016(9)$ \\
\hline $\mathrm{O} 3 \mathrm{~A}$ & $0.0272(10)$ & 0.0415 (12) & $0.0330(11)$ & $0.0049(9)$ & $0.0179(9)$ & $0.0043(9)$ \\
\hline $\mathrm{O} 4 \mathrm{~A}$ & $0.0351(11)$ & 0.0385 (12) & $0.0231(11)$ & $-0.0070(9)$ & $0.0154(9)$ & $-0.0027(9)$ \\
\hline O1B & $0.0224(10)$ & 0.0533 (13) & $0.0322(11)$ & $0.0001(9)$ & $0.0144(9)$ & $0.0041(10)$ \\
\hline $\mathrm{O} 2 \mathrm{~A}$ & 0.0475 (13) & 0.0464 (13) & $0.0253(11)$ & $0.0061(11)$ & $0.0167(10)$ & $0.0088(10)$ \\
\hline $\mathrm{O} 2 \mathrm{~B}$ & $0.0575(14)$ & 0.0363 & $0.0260(11)$ & $-0.0051(11)$ & $0.0150(11)$ & $-0.0077(10)$ \\
\hline $\mathrm{N} 1 \mathrm{~N}$ & $0.0234(11)$ & $0.0370(13)$ & $0.0229(12)$ & $0.0010(10)$ & $0.0150(10)$ & $-0.0003(10)$ \\
\hline N1M & $0.0289(12)$ & $0.0322(13)$ & $0.0300(13)$ & $-0.0029(10)$ & $0.0183(11)$ & 0.0008 (11) \\
\hline $\mathrm{C} 21 \mathrm{~B}$ & 0.0225 & $0.0314(15)$ & $0.0220(14)$ & $0.0021(11)$ & $0.0128(12)$ & $-0.0002(12)$ \\
\hline $\mathrm{C} 7 \mathrm{~B}$ & $0.0239(14)$ & $0.0356(16)$ & $0.0244(14)$ & $0.0001(12)$ & $0.0149(12)$ & $0.0038(12)$ \\
\hline C19B & $0.0260(13)$ & 0.0298 (14) & $0.0245(14)$ & $0.0019(11)$ & $0.0163(12)$ & $0.0014(12)$ \\
\hline $\mathrm{C} 20 \mathrm{~B}$ & $0.0209(12)$ & $0.0354(15)$ & $0.0238(14)$ & $-0.0023(11)$ & $0.0142(11)$ & $-0.0015(12)$ \\
\hline C7A & $0.0311(14)$ & $0.0264(14)$ & $0.0281(15)$ & 0.0017 (11) & $0.0189(13)$ & $0.0057(12)$ \\
\hline $\mathrm{C} 20 \mathrm{~A}$ & $0.0335(15)$ & $0.0341(16)$ & $0.0272(15)$ & $-0.0013(12)$ & $0.0211(13)$ & $-0.0006(12)$ \\
\hline $\mathrm{C} 13 \mathrm{~A}$ & $0.0270(14)$ & 0.0357 (16) & $0.0254(15)$ & 0.0029 (12) & $0.0154(12)$ & $-0.0005(12)$ \\
\hline C18B & $0.0246(14)$ & $0.0431(17)$ & $0.0253(15)$ & $-0.0044(12)$ & $0.0145(12)$ & $-0.0050(13)$ \\
\hline C13B & 0.0245 (13) & $0.0370(16)$ & 0.0237 (14) & $-0.0006(12)$ & $0.0149(12)$ & $-0.0012(12)$ \\
\hline
\end{tabular}




\begin{tabular}{|c|c|c|c|c|c|c|}
\hline C14A & $0.0325(15)$ & $0.0356(17)$ & $0.0260(15)$ & $0.0015(12)$ & $0.0153(13)$ & $-0.0014(12)$ \\
\hline $\mathrm{C} 2 \mathrm{~B}$ & $0.0388(16)$ & $0.0340(16)$ & $0.0328(17)$ & $0.0039(13)$ & $0.0194(14)$ & $0.0067(13)$ \\
\hline $\mathrm{C} 6 \mathrm{~B}$ & $0.0234(13)$ & $0.0373(16)$ & $0.0242(14)$ & $0.0026(11)$ & $0.0162(12)$ & $0.0030(12)$ \\
\hline $\mathrm{C} 4 \mathrm{~N}$ & $0.0265(14)$ & $0.0424(17)$ & $0.0233(14)$ & $-0.0013(12)$ & $0.0164(12)$ & $-0.0026(12)$ \\
\hline $\mathrm{C} 12 \mathrm{~N}$ & $0.0372(15)$ & $0.0393(17)$ & $0.0274(15)$ & $0.0031(13)$ & $0.0220(14)$ & 0.0009 (13) \\
\hline $\mathrm{C} 7 \mathrm{~N}$ & $0.0285(14)$ & $0.0328(15)$ & $0.0259(15)$ & $0.0023(12)$ & $0.0172(12)$ & $0.0013(12)$ \\
\hline C19A & $0.0312(15)$ & $0.0256(14)$ & $0.0289(15)$ & $0.0003(11)$ & $0.0193(13)$ & 0.0004 (12) \\
\hline $\mathrm{C} 9 \mathrm{~B}$ & $0.0331(15)$ & $0.0324(16)$ & $0.0247(15)$ & $-0.0011(12)$ & $0.0134(13)$ & $0.0002(12)$ \\
\hline C14B & $0.0252(14)$ & $0.0386(17)$ & $0.0248(15)$ & $-0.0007(12)$ & $0.0139(12)$ & $-0.0038(13)$ \\
\hline $\mathrm{C} 21 \mathrm{~A}$ & $0.0330(14)$ & $0.0255(14)$ & $0.0240(15)$ & $-0.0039(12)$ & 0.0164 (13) & $-0.0005(11)$ \\
\hline $\mathrm{C} 8 \mathrm{~A}$ & $0.0287(14)$ & $0.0277(15)$ & $0.0234(14)$ & $0.0028(11)$ & $0.0158(12)$ & $0.0052(11)$ \\
\hline C15A & $0.0369(16)$ & $0.0333(17)$ & $0.0369(17)$ & 0.0041 (13) & $0.0208(15)$ & 0.0027 (13) \\
\hline $\mathrm{C} 22 \mathrm{~B}$ & $0.0321(15)$ & $0.053(2)$ & $0.0322(16)$ & 0.0079 (14) & $0.0243(14)$ & $0.0034(14)$ \\
\hline $\mathrm{C} 4 \mathrm{~B}$ & $0.0289(14)$ & $0.0337(16)$ & $0.0254(15)$ & $0.0006(12)$ & $0.0133(12)$ & $0.0035(12)$ \\
\hline $\mathrm{C} 5 \mathrm{~N}$ & $0.0250(14)$ & 0.0484 (19) & $0.0299(16)$ & $0.0006(13)$ & 0.0154 (13) & $-0.0023(14)$ \\
\hline $\mathrm{C} 12 \mathrm{~B}$ & $0.0315(15)$ & $0.0343(17)$ & $0.0225(15)$ & $-0.0041(13)$ & 0.0135 (13) & $-0.0026(13)$ \\
\hline $\mathrm{C} 8 \mathrm{~B}$ & $0.0241(13)$ & $0.0301(15)$ & $0.0219(14)$ & $-0.0003(11)$ & $0.0121(12)$ & $0.0002(11)$ \\
\hline C6A & $0.0264(14)$ & $0.0334(16)$ & $0.0269(14)$ & $-0.0015(12)$ & $0.0151(12)$ & $0.0012(12)$ \\
\hline C17B & $0.0305(15)$ & $0.0482(19)$ & $0.0240(15)$ & 0.0059 (14) & $0.0136(13)$ & 0.0029 (14) \\
\hline $\mathrm{C} 11 \mathrm{~N}$ & $0.0443(17)$ & $0.0383(18)$ & $0.0351(17)$ & $0.0022(14)$ & $0.0281(15)$ & $-0.0013(14)$ \\
\hline $\mathrm{C} 7 \mathrm{M}$ & $0.0332(15)$ & $0.0341(16)$ & $0.0355(17)$ & $-0.0053(13)$ & $0.0229(14)$ & $-0.0041(13)$ \\
\hline $\mathrm{C} 4 \mathrm{M}$ & $0.0355(16)$ & $0.0337(17)$ & $0.0407(18)$ & $-0.0040(13)$ & $0.0255(15)$ & $0.0031(14)$ \\
\hline
\end{tabular}




\begin{tabular}{|c|c|c|c|c|c|c|}
\hline C5B & $0.0282(14)$ & $0.0401(17)$ & $0.0282(15)$ & 0.0015 (12) & $0.0166(13)$ & $0.0051(13)$ \\
\hline C16B & 0.0356 (16) & $0.0402(18)$ & 0.0345 (17) & 0.0107 (14) & $0.0213(14)$ & $0.0078(14)$ \\
\hline $\mathrm{C} 12 \mathrm{~A}$ & $0.0317(15)$ & $0.0406(17)$ & $0.0253(16)$ & $0.0044(13)$ & $0.0180(13)$ & $0.0042(13)$ \\
\hline C18A & $0.0380(16)$ & 0.0457 (19) & $0.0288(16)$ & 0.0058 (14) & $0.0212(14)$ & $-0.0005(14)$ \\
\hline $\mathrm{C} 8 \mathrm{M}$ & 0.0397 (17) & $0.0377(17)$ & $0.0425(18)$ & $-0.0025(13)$ & $0.0289(15)$ & $-0.0015(14)$ \\
\hline $\mathrm{C} 12 \mathrm{M}$ & 0.0424 (17) & 0.0375 (17) & $0.0461(19)$ & $-0.0024(14)$ & $0.0294(16)$ & $0.0025(15)$ \\
\hline $\mathrm{C} 1 \mathrm{~B}$ & $0.0432(19)$ & 0.057 (2) & $0.040(2)$ & 0.0027 (16) & $0.0232(16)$ & 0.0098 (17) \\
\hline C9A & $0.0403(16)$ & $0.0255(15)$ & $0.0344(16)$ & $0.0036(13)$ & $0.0230(14)$ & $0.0083(12)$ \\
\hline $\mathrm{C} 3 \mathrm{~N}$ & $0.0323(15)$ & 0.0475 (19) & 0.0308 (16) & $0.0036(14)$ & $0.0196(14)$ & $-0.0057(14)$ \\
\hline C15B & $0.0307(15)$ & $0.0358(16)$ & 0.0347 (17) & $0.0021(12)$ & 0.0194 (14) & $0.0006(13)$ \\
\hline $\mathrm{C} 2 \mathrm{~N}$ & 0.0444 (19) & $0.054(2)$ & $0.0382(18)$ & $-0.0016(16)$ & $0.0249(16)$ & $-0.0139(16)$ \\
\hline C5A & $0.0343(16)$ & $0.0353(17)$ & $0.0333(16)$ & $-0.0074(12)$ & $0.0210(14)$ & $-0.0016(13)$ \\
\hline $\mathrm{C} 17 \mathrm{~A}$ & $0.0461(18)$ & 0.047 (2) & 0.0365 (18) & $-0.0006(16)$ & $0.0239(16)$ & $-0.0127(16)$ \\
\hline C10B & 0.0536 (19) & $0.0338(17)$ & $0.0351(18)$ & $0.0106(15)$ & $0.0245(16)$ & $-0.0002(14)$ \\
\hline $\mathrm{C} 3 \mathrm{~B}$ & 0.0411 (17) & $0.0342(17)$ & 0.0332 (17) & 0.0008 (14) & $0.0175(15)$ & $0.0056(14)$ \\
\hline $\mathrm{C} 8 \mathrm{~N}$ & 0.055 (2) & 0.0409 (18) & 0.0367 (17) & $-0.0043(15)$ & $0.0351(16)$ & $-0.0005(15)$ \\
\hline $\mathrm{C} 10 \mathrm{~N}$ & $0.061(2)$ & $0.0368(18)$ & $0.052(2)$ & $-0.0037(16)$ & 0.0429 (19) & $-0.0029(16)$ \\
\hline $\mathrm{C} 3 \mathrm{M}$ & $0.0371(16)$ & $0.0376(18)$ & $0.051(2)$ & $-0.0075(14)$ & $0.0274(16)$ & $-0.0067(15)$ \\
\hline $\mathrm{C} 5 \mathrm{M}$ & 0.0348 (16) & 0.0365 (18) & $0.0394(18)$ & $-0.0039(14)$ & $0.0175(15)$ & $0.0031(15)$ \\
\hline $\mathrm{C} 9 \mathrm{~N}$ & $0.082(3)$ & $0.038(2)$ & $0.054(2)$ & $-0.0039(17)$ & $0.053(2)$ & $0.0026(16)$ \\
\hline C23B & 0.0462 (19) & 0.058 (2) & $0.0356(18)$ & 0.0199 (17) & $0.0238(16)$ & $0.0054(16)$ \\
\hline $\mathrm{C} 23 \mathrm{~A}$ & $0.0329(16)$ & 0.0419 (19) & $0.0356(18)$ & 0.0061 (14) & $0.0138(15)$ & $0.0041(15)$ \\
\hline
\end{tabular}




\begin{tabular}{|c|c|c|c|c|c|c|}
\hline $\mathrm{C} 6 \mathrm{~N}$ & $0.0267(15)$ & $0.062(2)$ & $0.0303(16)$ & $-0.0050(15)$ & $0.0139(14)$ & $-0.0060(16)$ \\
\hline $\mathrm{C} 24 \mathrm{~A}$ & $0.0412(18)$ & $0.0388(18)$ & $0.050(2)$ & $0.0032(15)$ & $0.0283(16)$ & $-0.0030(16)$ \\
\hline $\mathrm{C} 4 \mathrm{~A}$ & $0.0450(17)$ & $0.0283(16)$ & $0.0402(18)$ & $-0.0011(13)$ & $0.0269(15)$ & $0.0016(13)$ \\
\hline C16A & $0.0410(17)$ & $0.0351(18)$ & $0.047(2)$ & $0.0012(14)$ & $0.0255(16)$ & $-0.0076(15)$ \\
\hline $\mathrm{C} 9 \mathrm{M}$ & $0.0476(18)$ & $0.050(2)$ & 0.055 (2) & $-0.0035(16)$ & $0.0394(18)$ & $-0.0101(17)$ \\
\hline $\mathrm{C} 1 \mathrm{~N}$ & 0.0416 (19) & 0.058 (2) & 0.0385 (19) & $-0.0093(16)$ & $0.0244(16)$ & $-0.0155(16)$ \\
\hline $\mathrm{C} 22 \mathrm{~A}$ & 0.0269 (14) & $0.0435(18)$ & 0.0325 (17) & 0.0009 (13) & $0.0146(13)$ & $0.0040(14)$ \\
\hline C24B & 0.069 (2) & $0.044(2)$ & $0.052(2)$ & $0.0196(17)$ & $0.044(2)$ & $0.0051(17)$ \\
\hline C11A & $0.0521(19)$ & $0.0386(18)$ & 0.0443 (19) & $0.0012(15)$ & $0.0338(17)$ & $0.0103(15)$ \\
\hline C11B & 0.0472 (19) & 0.0387 (19) & $0.0322(18)$ & $-0.0095(15)$ & $0.0142(15)$ & $0.0038(14)$ \\
\hline $\mathrm{C} 2 \mathrm{M}$ & $0.051(2)$ & $0.0387(19)$ & $0.067(2)$ & $-0.0112(16)$ & $0.036(2)$ & $-0.0036(18)$ \\
\hline $\mathrm{C} 10 \mathrm{~A}$ & $0.0474(18)$ & 0.0337 (17) & $0.0424(19)$ & $0.0081(14)$ & $0.0269(16)$ & $0.0099(15)$ \\
\hline $\mathrm{C} 10 \mathrm{M}$ & 0.055 (2) & $0.0357(18)$ & $0.074(3)$ & $-0.0067(15)$ & 0.048 (2) & $-0.0084(17)$ \\
\hline $\mathrm{C} 1 \mathrm{M}$ & $0.051(2)$ & $0.0360(19)$ & 0.067 (3) & $-0.0063(16)$ & $0.032(2)$ & $0.0079(17)$ \\
\hline $\mathrm{C} 25 \mathrm{~B}$ & $0.062(2)$ & 0.055 (2) & $0.044(2)$ & $0.0161(19)$ & 0.0313 (19) & $0.0033(18)$ \\
\hline $\mathrm{C} 11 \mathrm{M}$ & $0.056(2)$ & 0.0355 (18) & $0.067(2)$ & $-0.0035(15)$ & $0.044(2)$ & $0.0004(16)$ \\
\hline C6M & 0.049 (2) & $0.044(2)$ & $0.056(2)$ & $-0.0034(16)$ & $0.0240(18)$ & $0.0139(18)$ \\
\hline $\mathrm{C} 26 \mathrm{~A}$ & $0.050(2)$ & $0.044(2)$ & $0.063(2)$ & $0.0028(17)$ & $0.034(2)$ & $0.0021(18)$ \\
\hline $\mathrm{C} 27 \mathrm{~B}$ & $0.040(4)$ & $0.040(11)$ & $0.081(7)$ & $0.012(6)$ & $0.033(4)$ & $-0.005(6)$ \\
\hline $\mathrm{C} 28 \mathrm{~A}$ & $0.066(2)$ & $0.065(3)$ & $0.046(2)$ & $0.021(2)$ & $0.035(2)$ & 0.007 (2) \\
\hline $\mathrm{C} 3 \mathrm{~A}$ & 0.059 (2) & $0.0322(18)$ & 0.059 (2) & $-0.0027(16)$ & $0.034(2)$ & 0.0017 (16) \\
\hline C27A & 0.054 (2) & 0.063 (3) & $0.046(2)$ & $0.0100(19)$ & $0.0334(18)$ & $0.0042(18)$ \\
\hline
\end{tabular}




\begin{tabular}{|l|l|l|l|l|l|l|}
\hline C26B & $0.142(5)$ & $0.051(3)$ & $0.079(3)$ & $0.000(3)$ & $0.085(3)$ & $-0.006(2)$ \\
\hline C2A & $0.074(3)$ & $0.040(2)$ & $0.055(2)$ & $0.0019(19)$ & $0.032(2)$ & $0.0024(18)$ \\
\hline C25A & $0.058(2)$ & $0.059(2)$ & $0.044(2)$ & $-0.0048(18)$ & $0.0321(19)$ & $0.0004(17)$ \\
\hline C29A & $0.071(3)$ & $0.069(3)$ & $0.073(3)$ & $0.018(2)$ & $0.049(2)$ & $0.011(2)$ \\
\hline C1A & $0.098(3)$ & $0.042(2)$ & $0.062(3)$ & $0.018(2)$ & $0.046(3)$ & $0.002(2)$ \\
\hline C28B & $0.048(6)$ & $0.048(7)$ & $0.048(6)$ & $0.013(5)$ & $0.024(5)$ & $0.000(5)$ \\
\hline C29B & $0.070(7)$ & $0.058(7)$ & $0.074(7)$ & $0.020(5)$ & $0.046(6)$ & $0.003(5)$ \\
\hline C29C & $0.088(9)$ & $0.050(6)$ & $0.071(7)$ & $0.023(5)$ & $0.063(7)$ & $0.006(5)$ \\
\hline C28C & $0.055(8)$ & $0.037(7)$ & $0.117(12)$ & $0.004(5)$ & $0.059(8)$ & $-0.006(6)$ \\
\hline C28D & $0.048(15)$ & $0.070(18)$ & $0.052(14)$ & $0.015(12)$ & $0.032(13)$ & $-0.018(12)$ \\
\hline C29D & $0.039(13)$ & $0.13(3)$ & $0.069(18)$ & $0.008(15)$ & $0.040(14)$ & $-0.011(17)$ \\
\hline C27C & $0.040(4)$ & $0.040(11)$ & $0.081(7)$ & $0.012(6)$ & $0.033(4)$ & $-0.005(6)$ \\
\hline C27D & $0.040(4)$ & $0.040(11)$ & $0.081(7)$ & $0.012(6)$ & $0.033(4)$ & $-0.005(6)$ \\
\hline
\end{tabular}

Table S11. Geometric parameters $\left(\AA{ }^{\circ},{ }^{\circ}\right)$ for 21

\begin{tabular}{|l|l|l|l|}
\hline O3B-C19B & $1.259(3)$ & $\mathrm{C} 5 \mathrm{~N}-\mathrm{C} 6 \mathrm{~N}$ & $1.529(5)$ \\
\hline $\mathrm{O} 4 \mathrm{~B}-\mathrm{C} 21 \mathrm{~B}$ & $1.256(3)$ & $\mathrm{C} 12 \mathrm{~B}-\mathrm{C} 8 \mathrm{~B}$ & $1.555(4)$ \\
\hline $\mathrm{O} 1 \mathrm{~A}-\mathrm{C} 7 \mathrm{~A}$ & $1.217(4)$ & $\mathrm{C} 6 \mathrm{~A}-\mathrm{C} 5 \mathrm{~A}$ & $1.542(4)$ \\
\hline O3A-C19A & $1.265(3)$ & $\mathrm{C} 6 \mathrm{~A}-\mathrm{C} 22 \mathrm{~A}$ & $1.532(4)$ \\
\hline O4A-C21A & $1.262(3)$ & $\mathrm{C} 17 \mathrm{~B}-\mathrm{C} 16 \mathrm{~B}$ & $1.377(5)$ \\
\hline $\mathrm{O} 1 \mathrm{~B}-\mathrm{C} 7 \mathrm{~B}$ & $1.216(3)$ & $\mathrm{C} 11 \mathrm{~N}-\mathrm{C} 10 \mathrm{~N}$ & $1.522(5)$ \\
\hline O2A-C12A & $1.222(4)$ & $\mathrm{C} 7 \mathrm{M}-\mathrm{C} 8 \mathrm{M}$ & $1.522(4)$ \\
\hline
\end{tabular}




\begin{tabular}{|c|c|c|c|}
\hline $\mathrm{O} 2 \mathrm{~B}-\mathrm{C} 12 \mathrm{~B}$ & $1.212(4)$ & $\mathrm{C} 7 \mathrm{M}-\mathrm{C} 12 \mathrm{M}$ & $1.524(5)$ \\
\hline $\mathrm{N} 1 \mathrm{~N}-\mathrm{C} 4 \mathrm{~N}$ & $1.503(4)$ & $\mathrm{C} 4 \mathrm{M}-\mathrm{C} 3 \mathrm{M}$ & $1.514(5)$ \\
\hline $\mathrm{N} 1 \mathrm{~N}-\mathrm{C} 7 \mathrm{~N}$ & $1.497(4)$ & $\mathrm{C} 4 \mathrm{M}-\mathrm{C} 5 \mathrm{M}$ & $1.518(4)$ \\
\hline $\mathrm{N} 1 \mathrm{M}-\mathrm{C} 7 \mathrm{M}$ & $1.496(4)$ & $\mathrm{C} 16 \mathrm{~B}-\mathrm{C} 15 \mathrm{~B}$ & $1.381(5)$ \\
\hline $\mathrm{N} 1 \mathrm{M}-\mathrm{C} 4 \mathrm{M}$ & $1.495(4)$ & $\mathrm{C} 18 \mathrm{~A}-\mathrm{C} 17 \mathrm{~A}$ & $1.383(5)$ \\
\hline $\mathrm{C} 21 \mathrm{~B}-\mathrm{C} 20 \mathrm{~B}$ & $1.402(4)$ & $\mathrm{C} 8 \mathrm{M}-\mathrm{C} 9 \mathrm{M}$ & $1.524(5)$ \\
\hline $\mathrm{C} 21 \mathrm{~B}-\mathrm{C} 6 \mathrm{~B}$ & $1.531(4)$ & $\mathrm{C} 12 \mathrm{M}-\mathrm{C} 11 \mathrm{M}$ & $1.523(5)$ \\
\hline $\mathrm{C} 7 \mathrm{~B}-\mathrm{C} 6 \mathrm{~B}$ & $1.520(4)$ & $\mathrm{C} 9 \mathrm{~A}-\mathrm{C} 4 \mathrm{~A}$ & $1.565(5)$ \\
\hline $\mathrm{C} 7 \mathrm{~B}-\mathrm{C} 8 \mathrm{~B}$ & $1.513(4)$ & $\mathrm{C} 9 \mathrm{~A}-\mathrm{C} 11 \mathrm{~A}$ & $1.527(4)$ \\
\hline $\mathrm{C} 19 \mathrm{~B}-\mathrm{C} 20 \mathrm{~B}$ & $1.388(4)$ & $\mathrm{C} 9 \mathrm{~A}-\mathrm{C} 10 \mathrm{~A}$ & $1.534(4)$ \\
\hline $\mathrm{C} 19 \mathrm{~B}-\mathrm{C} 8 \mathrm{~B}$ & $1.543(4)$ & $\mathrm{C} 3 \mathrm{~N}-\mathrm{C} 2 \mathrm{~N}$ & $1.532(4)$ \\
\hline $\mathrm{C} 7 \mathrm{~A}-\mathrm{C} 8 \mathrm{~A}$ & $1.513(4)$ & $\mathrm{C} 2 \mathrm{~N}-\mathrm{C} 1 \mathrm{~N}$ & $1.515(5)$ \\
\hline $\mathrm{C} 7 \mathrm{~A}-\mathrm{C} 6 \mathrm{~A}$ & $1.518(4)$ & $\mathrm{C} 5 \mathrm{~A}-\mathrm{C} 4 \mathrm{~A}$ & $1.528(4)$ \\
\hline $\mathrm{C} 20 \mathrm{~A}-\mathrm{C} 19 \mathrm{~A}$ & $1.398(4)$ & $\mathrm{C} 17 \mathrm{~A}-\mathrm{C} 16 \mathrm{~A}$ & $1.378(5)$ \\
\hline $\mathrm{C} 20 \mathrm{~A}-\mathrm{C} 21 \mathrm{~A}$ & $1.400(4)$ & $\mathrm{C} 8 \mathrm{~N}-\mathrm{C} 9 \mathrm{~N}$ & $1.521(5)$ \\
\hline $\mathrm{C} 13 \mathrm{~A}-\mathrm{C} 14 \mathrm{~A}$ & $1.398(4)$ & $\mathrm{C} 10 \mathrm{~N}-\mathrm{C} 9 \mathrm{~N}$ & $1.517(5)$ \\
\hline $\mathrm{C} 13 \mathrm{~A}-\mathrm{C} 12 \mathrm{~A}$ & $1.502(4)$ & $\mathrm{C} 3 \mathrm{M}-\mathrm{C} 2 \mathrm{M}$ & $1.518(5)$ \\
\hline $\mathrm{C} 13 \mathrm{~A}-\mathrm{C} 18 \mathrm{~A}$ & $1.390(4)$ & $\mathrm{C} 5 \mathrm{M}-\mathrm{C} 6 \mathrm{M}$ & $1.522(5)$ \\
\hline $\mathrm{C} 18 \mathrm{~B}-\mathrm{C} 13 \mathrm{~B}$ & $1.399(4)$ & $\mathrm{C} 23 \mathrm{~B}-\mathrm{C} 24 \mathrm{~B}$ & $1.498(6)$ \\
\hline $\mathrm{C} 18 \mathrm{~B}-\mathrm{C} 17 \mathrm{~B}$ & $1.384(5)$ & $\mathrm{C} 23 \mathrm{~B}-\mathrm{C} 27 \mathrm{~B}$ & $1.541(12)$ \\
\hline $\mathrm{C} 13 \mathrm{~B}-\mathrm{C} 14 \mathrm{~B}$ & $1.395(4)$ & $\mathrm{C} 23 \mathrm{~B}-\mathrm{C} 27 \mathrm{C}$ & $1.569(14)$ \\
\hline $\mathrm{C} 13 \mathrm{~B}-\mathrm{C} 12 \mathrm{~B}$ & $1.501(4)$ & $\mathrm{C} 23 \mathrm{~B}-\mathrm{C} 27 \mathrm{D}$ & $1.560(17)$ \\
\hline
\end{tabular}




\begin{tabular}{|c|c|c|c|}
\hline $\mathrm{C} 14 \mathrm{~A}-\mathrm{C} 15 \mathrm{~A}$ & $1.384(5)$ & $\mathrm{C} 23 \mathrm{~A}-\mathrm{C} 24 \mathrm{~A}$ & $1.484(5)$ \\
\hline $\mathrm{C} 2 \mathrm{~B}-\mathrm{C} 1 \mathrm{~B}$ & $1.304(5)$ & $\mathrm{C} 23 \mathrm{~A}-\mathrm{C} 22 \mathrm{~A}$ & $1.542(5)$ \\
\hline $\mathrm{C} 2 \mathrm{~B}-\mathrm{C} 3 \mathrm{~B}$ & 1.499 (4) & $\mathrm{C} 23 \mathrm{~A}-\mathrm{C} 27 \mathrm{~A}$ & $1.570(5)$ \\
\hline $\mathrm{C} 6 \mathrm{~B}-\mathrm{C} 22 \mathrm{~B}$ & $1.526(4)$ & $\mathrm{C} 6 \mathrm{~N}-\mathrm{C} 1 \mathrm{~N}$ & $1.520(5)$ \\
\hline $\mathrm{C} 6 \mathrm{~B}-\mathrm{C} 5 \mathrm{~B}$ & $1.555(4)$ & $\mathrm{C} 24 \mathrm{~A}-\mathrm{C} 26 \mathrm{~A}$ & $1.321(5)$ \\
\hline $\mathrm{C} 4 \mathrm{~N}-\mathrm{C} 5 \mathrm{~N}$ & $1.523(4)$ & $\mathrm{C} 24 \mathrm{~A}-\mathrm{C} 25 \mathrm{~A}$ & $1.509(5)$ \\
\hline $\mathrm{C} 4 \mathrm{~N}-\mathrm{C} 3 \mathrm{~N}$ & $1.512(5)$ & $\mathrm{C} 4 \mathrm{~A}-\mathrm{C} 3 \mathrm{~A}$ & $1.541(5)$ \\
\hline $\mathrm{C} 12 \mathrm{~N}-\mathrm{C} 7 \mathrm{~N}$ & $1.529(4)$ & $\mathrm{C} 9 \mathrm{M}-\mathrm{C} 10 \mathrm{M}$ & $1.523(5)$ \\
\hline $\mathrm{C} 12 \mathrm{~N}-\mathrm{C} 11 \mathrm{~N}$ & $1.522(4)$ & $\mathrm{C} 24 \mathrm{~B}-\mathrm{C} 25 \mathrm{~B}$ & $1.497(5)$ \\
\hline $\mathrm{C} 7 \mathrm{~N}-\mathrm{C} 8 \mathrm{~N}$ & $1.517(4)$ & $\mathrm{C} 24 \mathrm{~B}-\mathrm{C} 26 \mathrm{~B}$ & $1.328(6)$ \\
\hline $\mathrm{C} 19 \mathrm{~A}-\mathrm{C} 8 \mathrm{~A}$ & $1.536(4)$ & $\mathrm{C} 2 \mathrm{M}-\mathrm{C} 1 \mathrm{M}$ & $1.527(5)$ \\
\hline $\mathrm{C} 9 \mathrm{~B}-\mathrm{C} 4 \mathrm{~B}$ & $1.573(4)$ & $\mathrm{C} 10 \mathrm{M}-\mathrm{C} 11 \mathrm{M}$ & $1.505(5)$ \\
\hline $\mathrm{C} 9 \mathrm{~B}-\mathrm{C} 8 \mathrm{~B}$ & $1.600(4)$ & $\mathrm{C} 1 \mathrm{M}-\mathrm{C} 6 \mathrm{M}$ & $1.521(6)$ \\
\hline $\mathrm{C} 9 \mathrm{~B}-\mathrm{C} 10 \mathrm{~B}$ & $1.533(5)$ & $\mathrm{C} 27 \mathrm{~B}-\mathrm{C} 28 \mathrm{~B}$ & $1.513(15)$ \\
\hline $\mathrm{C} 9 \mathrm{~B}-\mathrm{C} 11 \mathrm{~B}$ & $1.528(5)$ & $\mathrm{C} 28 \mathrm{~A}-\mathrm{C} 27 \mathrm{~A}$ & $1.470(6)$ \\
\hline $\mathrm{C} 14 \mathrm{~B}-\mathrm{C} 15 \mathrm{~B}$ & $1.384(5)$ & $\mathrm{C} 28 \mathrm{~A}-\mathrm{C} 29 \mathrm{~A}$ & $1.325(6)$ \\
\hline $\mathrm{C} 21 \mathrm{~A}-\mathrm{C} 6 \mathrm{~A}$ & $1.548(4)$ & $\mathrm{C} 3 \mathrm{~A}-\mathrm{C} 2 \mathrm{~A}$ & $1.471(6)$ \\
\hline $\mathrm{C} 8 \mathrm{~A}-\mathrm{C} 12 \mathrm{~A}$ & $1.549(4)$ & $\mathrm{C} 2 \mathrm{~A}-\mathrm{C} 1 \mathrm{~A}$ & $1.318(7)$ \\
\hline $\mathrm{C} 8 \mathrm{~A}-\mathrm{C} 9 \mathrm{~A}$ & $1.604(4)$ & $\mathrm{C} 28 \mathrm{~B}-\mathrm{C} 29 \mathrm{~B}$ & $1.287(12)$ \\
\hline $\mathrm{C} 15 \mathrm{~A}-\mathrm{C} 16 \mathrm{~A}$ & $1.378(5)$ & $\mathrm{C} 29 \mathrm{C}-\mathrm{C} 28 \mathrm{C}$ & $1.279(13)$ \\
\hline $\mathrm{C} 22 \mathrm{~B}-\mathrm{C} 23 \mathrm{~B}$ & $1.538(5)$ & $\mathrm{C} 28 \mathrm{C}-\mathrm{C} 27 \mathrm{C}$ & $1.526(15)$ \\
\hline $\mathrm{C} 4 \mathrm{~B}-\mathrm{C} 5 \mathrm{~B}$ & $1.524(4)$ & $\mathrm{C} 28 \mathrm{D}-\mathrm{C} 29 \mathrm{D}$ & $1.286(18)$ \\
\hline
\end{tabular}




\begin{tabular}{|c|c|c|c|}
\hline $\mathrm{C} 4 \mathrm{~B}-\mathrm{C} 3 \mathrm{~B}$ & $1.540(4)$ & $\mathrm{C} 28 \mathrm{D}-\mathrm{C} 27 \mathrm{D}$ & $1.516(18)$ \\
\hline $\mathrm{C} 7 \mathrm{~N}-\mathrm{N} 1 \mathrm{~N}-\mathrm{C} 4 \mathrm{~N}$ & $117.7(2)$ & $\mathrm{C} 5 \mathrm{~A}-\mathrm{C} 6 \mathrm{~A}-\mathrm{C} 21 \mathrm{~A}$ & $106.8(2)$ \\
\hline $\mathrm{C} 4 \mathrm{M}-\mathrm{N} 1 \mathrm{M}-\mathrm{C} 7 \mathrm{M}$ & $117.9(2)$ & $\mathrm{C} 22 \mathrm{~A}-\mathrm{C} 6 \mathrm{~A}-\mathrm{C} 21 \mathrm{~A}$ & $113.8(2)$ \\
\hline $\mathrm{O} 4 \mathrm{~B}-\mathrm{C} 21 \mathrm{~B}-\mathrm{C} 20 \mathrm{~B}$ & $124.2(2)$ & $\mathrm{C} 22 \mathrm{~A}-\mathrm{C} 6 \mathrm{~A}-\mathrm{C} 5 \mathrm{~A}$ & $108.5(2)$ \\
\hline $\mathrm{O} 4 \mathrm{~B}-\mathrm{C} 21 \mathrm{~B}-\mathrm{C} 6 \mathrm{~B}$ & $116.3(2)$ & $\mathrm{C} 16 \mathrm{~B}-\mathrm{C} 17 \mathrm{~B}-\mathrm{C} 18 \mathrm{~B}$ & $120.9(3)$ \\
\hline $\mathrm{C} 20 \mathrm{~B}-\mathrm{C} 21 \mathrm{~B}-\mathrm{C} 6 \mathrm{~B}$ & $119.4(2)$ & $\mathrm{C} 12 \mathrm{~N}-\mathrm{C} 11 \mathrm{~N}-\mathrm{C} 10 \mathrm{~N}$ & 110.3 \\
\hline $\mathrm{O} 1 \mathrm{~B}-\mathrm{C} 7 \mathrm{~B}-\mathrm{C} 6 \mathrm{~B}$ & $123.7(2)$ & $\mathrm{N} 1 \mathrm{M}-\mathrm{C} 7 \mathrm{M}-\mathrm{C} 8 \mathrm{M}$ & $111.7(2)$ \\
\hline $\mathrm{O} 1 \mathrm{~B}-\mathrm{C} 7 \mathrm{~B}-\mathrm{C} 8 \mathrm{~B}$ & $121.5(3)$ & $\mathrm{N} 1 \mathrm{M}-\mathrm{C} 7 \mathrm{M}-\mathrm{C} 12 \mathrm{M}$ & $107.3(2)$ \\
\hline $\mathrm{C} 8 \mathrm{~B}-\mathrm{C} 7 \mathrm{~B}-\mathrm{C} 6 \mathrm{~B}$ & $114.1(2)$ & $\mathrm{C} 8 \mathrm{M}-\mathrm{C} 7 \mathrm{M}-\mathrm{C} 12 \mathrm{M}$ & $111.6(3)$ \\
\hline $\mathrm{O} 3 \mathrm{~B}-\mathrm{C} 19 \mathrm{~B}-\mathrm{C} 20 \mathrm{~B}$ & $125.3(3)$ & $\mathrm{N} 1 \mathrm{M}-\mathrm{C} 4 \mathrm{M}-\mathrm{C} 3 \mathrm{M}$ & $108.2(3)$ \\
\hline $\mathrm{O} 3 \mathrm{~B}-\mathrm{C} 19 \mathrm{~B}-\mathrm{C} 8 \mathrm{~B}$ & $115.3(2)$ & $\mathrm{N} 1 \mathrm{M}-\mathrm{C} 4 \mathrm{M}-\mathrm{C} 5 \mathrm{M}$ & $111.5(2)$ \\
\hline $\mathrm{C} 20 \mathrm{~B}-\mathrm{C} 19 \mathrm{~B}-\mathrm{C} 8 \mathrm{~B}$ & $119.4(2)$ & $\mathrm{C} 3 \mathrm{M}-\mathrm{C} 4 \mathrm{M}-\mathrm{C} 5 \mathrm{M}$ & $112.1(3)$ \\
\hline $\mathrm{C} 19 \mathrm{~B}-\mathrm{C} 20 \mathrm{~B}-\mathrm{C} 21 \mathrm{~B}$ & $123.9(2)$ & $\mathrm{C} 4 \mathrm{~B}-\mathrm{C} 5 \mathrm{~B}-\mathrm{C} 6 \mathrm{~B}$ & $113.8(2)$ \\
\hline $\mathrm{O} 1 \mathrm{~A}-\mathrm{C} 7 \mathrm{~A}-\mathrm{C} 8 \mathrm{~A}$ & $121.7(3)$ & $\mathrm{C} 17 \mathrm{~B}-\mathrm{C} 16 \mathrm{~B}-\mathrm{C} 15 \mathrm{~B}$ & $119.6(3)$ \\
\hline $\mathrm{O} 1 \mathrm{~A}-\mathrm{C} 7 \mathrm{~A}-\mathrm{C} 6 \mathrm{~A}$ & $123.2(3)$ & $\mathrm{O} 2 \mathrm{~A}-\mathrm{C} 12 \mathrm{~A}-\mathrm{C} 13 \mathrm{~A}$ & $118.6(3)$ \\
\hline $\mathrm{C} 8 \mathrm{~A}-\mathrm{C} 7 \mathrm{~A}-\mathrm{C} 6 \mathrm{~A}$ & $114.6(2)$ & $\mathrm{O} 2 \mathrm{~A}-\mathrm{C} 12 \mathrm{~A}-\mathrm{C} 8 \mathrm{~A}$ & $122.1(3)$ \\
\hline $\mathrm{C} 19 \mathrm{~A}-\mathrm{C} 20 \mathrm{~A}-\mathrm{C} 21 \mathrm{~A}$ & $124.1(3)$ & $\mathrm{C} 13 \mathrm{~A}-\mathrm{C} 12 \mathrm{~A}-\mathrm{C} 8 \mathrm{~A}$ & $119.3(2)$ \\
\hline $\mathrm{C} 14 \mathrm{~A}-\mathrm{C} 13 \mathrm{~A}-\mathrm{C} 12 \mathrm{~A}$ & $123.8(3)$ & $\mathrm{C} 17 \mathrm{~A}-\mathrm{C} 18 \mathrm{~A}-\mathrm{C} 13 \mathrm{~A}$ & $120.3(3)$ \\
\hline $\mathrm{C} 18 \mathrm{~A}-\mathrm{C} 13 \mathrm{~A}-\mathrm{C} 14 \mathrm{~A}$ & $118.8(3)$ & $\mathrm{C} 7 \mathrm{M}-\mathrm{C} 8 \mathrm{M}-\mathrm{C} 9 \mathrm{M}$ & $110.6(3)$ \\
\hline $\mathrm{C} 18 \mathrm{~A}-\mathrm{C} 13 \mathrm{~A}-\mathrm{C} 12 \mathrm{~A}$ & $117.4(3)$ & $\mathrm{C} 11 \mathrm{M}-\mathrm{C} 12 \mathrm{M}-\mathrm{C} 7 \mathrm{M}$ & $110.8(3)$ \\
\hline $\mathrm{C} 17 \mathrm{~B}-\mathrm{C} 18 \mathrm{~B}-\mathrm{C} 13 \mathrm{~B}$ & $120.1(3)$ & $\mathrm{C} 4 \mathrm{~A}-\mathrm{C} 9 \mathrm{~A}-\mathrm{C} 8 \mathrm{~A}$ & $108.0(2)$ \\
\hline
\end{tabular}




\begin{tabular}{|c|c|c|c|}
\hline $\mathrm{C} 18 \mathrm{~B}-\mathrm{C} 13 \mathrm{~B}-\mathrm{C} 12 \mathrm{~B}$ & $117.2(3)$ & $\mathrm{C} 11 \mathrm{~A}-\mathrm{C} 9 \mathrm{~A}-\mathrm{C} 8 \mathrm{~A}$ & $109.7(3)$ \\
\hline $\mathrm{C} 14 \mathrm{~B}-\mathrm{C} 13 \mathrm{~B}-\mathrm{C} 18 \mathrm{~B}$ & $118.4(3)$ & $\mathrm{C} 11 \mathrm{~A}-\mathrm{C} 9 \mathrm{~A}-\mathrm{C} 4 \mathrm{~A}$ & $111.0(3)$ \\
\hline $\mathrm{C} 14 \mathrm{~B}-\mathrm{C} 13 \mathrm{~B}-\mathrm{C} 12 \mathrm{~B}$ & $124.4(3)$ & $\mathrm{C} 11 \mathrm{~A}-\mathrm{C} 9 \mathrm{~A}-\mathrm{C} 10 \mathrm{~A}$ & $109.5(3)$ \\
\hline $\mathrm{C} 15 \mathrm{~A}-\mathrm{C} 14 \mathrm{~A}-\mathrm{C} 13 \mathrm{~A}$ & $120.2(3)$ & $\mathrm{C} 10 \mathrm{~A}-\mathrm{C} 9 \mathrm{~A}-\mathrm{C} 8 \mathrm{~A}$ & $110.2(2)$ \\
\hline $\mathrm{C} 1 \mathrm{~B}-\mathrm{C} 2 \mathrm{~B}-\mathrm{C} 3 \mathrm{~B}$ & $125.8(3)$ & $\mathrm{C} 10 \mathrm{~A}-\mathrm{C} 9 \mathrm{~A}-\mathrm{C} 4 \mathrm{~A}$ & $108.5(3)$ \\
\hline $\mathrm{C} 21 \mathrm{~B}-\mathrm{C} 6 \mathrm{~B}-\mathrm{C} 5 \mathrm{~B}$ & $105.3(2)$ & $\mathrm{C} 4 \mathrm{~N}-\mathrm{C} 3 \mathrm{~N}-\mathrm{C} 2 \mathrm{~N}$ & $111.6(3)$ \\
\hline $\mathrm{C} 7 \mathrm{~B}-\mathrm{C} 6 \mathrm{~B}-\mathrm{C} 21 \mathrm{~B}$ & $113.7(2)$ & $\mathrm{C} 16 \mathrm{~B}-\mathrm{C} 15 \mathrm{~B}-\mathrm{C} 14 \mathrm{~B}$ & $120.1(3)$ \\
\hline $\mathrm{C} 7 \mathrm{~B}-\mathrm{C} 6 \mathrm{~B}-\mathrm{C} 22 \mathrm{~B}$ & $112.0(2)$ & $\mathrm{C} 1 \mathrm{~N}-\mathrm{C} 2 \mathrm{~N}-\mathrm{C} 3 \mathrm{~N}$ & $111.3(3)$ \\
\hline $\mathrm{C} 7 \mathrm{~B}-\mathrm{C} 6 \mathrm{~B}-\mathrm{C} 5 \mathrm{~B}$ & $101.1(2)$ & $\mathrm{C} 4 \mathrm{~A}-\mathrm{C} 5 \mathrm{~A}-\mathrm{C} 6 \mathrm{~A}$ & $113.4(2)$ \\
\hline $\mathrm{C} 22 \mathrm{~B}-\mathrm{C} 6 \mathrm{~B}-\mathrm{C} 21 \mathrm{~B}$ & $112.3(2)$ & $\mathrm{C} 16 \mathrm{~A}-\mathrm{C} 17 \mathrm{~A}-\mathrm{C} 18 \mathrm{~A}$ & $120.5(3)$ \\
\hline $\mathrm{C} 22 \mathrm{~B}-\mathrm{C} 6 \mathrm{~B}-\mathrm{C} 5 \mathrm{~B}$ & $111.7(2)$ & $\mathrm{C} 2 \mathrm{~B}-\mathrm{C} 3 \mathrm{~B}-\mathrm{C} 4 \mathrm{~B}$ & $112.2(3)$ \\
\hline $\mathrm{N} 1 \mathrm{~N}-\mathrm{C} 4 \mathrm{~N}-\mathrm{C} 5 \mathrm{~N}$ & $111.5(2)$ & $\mathrm{C} 7 \mathrm{~N}-\mathrm{C} 8 \mathrm{~N}-\mathrm{C} 9 \mathrm{~N}$ & $110.3(3)$ \\
\hline $\mathrm{N} 1 \mathrm{~N}-\mathrm{C} 4 \mathrm{~N}-\mathrm{C} 3 \mathrm{~N}$ & $107.5(2)$ & $\mathrm{C} 9 \mathrm{~N}-\mathrm{C} 10 \mathrm{~N}-\mathrm{C} 11 \mathrm{~N}$ & $110.6(3)$ \\
\hline $\mathrm{C} 3 \mathrm{~N}-\mathrm{C} 4 \mathrm{~N}-\mathrm{C} 5 \mathrm{~N}$ & $111.8(3)$ & $\mathrm{C} 4 \mathrm{M}-\mathrm{C} 3 \mathrm{M}-\mathrm{C} 2 \mathrm{M}$ & $110.9(3)$ \\
\hline $\mathrm{C} 11 \mathrm{~N}-\mathrm{C} 12 \mathrm{~N}-\mathrm{C} 7 \mathrm{~N}$ & $110.8(3)$ & $\mathrm{C} 4 \mathrm{M}-\mathrm{C} 5 \mathrm{M}-\mathrm{C} 6 \mathrm{M}$ & $109.9(3)$ \\
\hline $\mathrm{N} 1 \mathrm{~N}-\mathrm{C} 7 \mathrm{~N}-\mathrm{C} 12 \mathrm{~N}$ & $108.5(2)$ & $\mathrm{C} 10 \mathrm{~N}-\mathrm{C} 9 \mathrm{~N}-\mathrm{C} 8 \mathrm{~N}$ & $111.6(3)$ \\
\hline $\mathrm{N} 1 \mathrm{~N}-\mathrm{C} 7 \mathrm{~N}-\mathrm{C} 8 \mathrm{~N}$ & $111.9(2)$ & $\mathrm{C} 22 \mathrm{~B}-\mathrm{C} 23 \mathrm{~B}-\mathrm{C} 27 \mathrm{~B}$ & $114.4(7)$ \\
\hline $\mathrm{C} 8 \mathrm{~N}-\mathrm{C} 7 \mathrm{~N}-\mathrm{C} 12 \mathrm{~N}$ & $111.1(2)$ & $\mathrm{C} 22 \mathrm{~B}-\mathrm{C} 23 \mathrm{~B}-\mathrm{C} 27 \mathrm{C}$ & $100.3(7)$ \\
\hline $\mathrm{O} 3 \mathrm{~A}-\mathrm{C} 19 \mathrm{~A}-\mathrm{C} 20 \mathrm{~A}$ & $124.7(3)$ & $\mathrm{C} 22 \mathrm{~B}-\mathrm{C} 23 \mathrm{~B}-\mathrm{C} 27 \mathrm{D}$ & $118(2)$ \\
\hline $\mathrm{O} 3 \mathrm{~A}-\mathrm{C} 19 \mathrm{~A}-\mathrm{C} 8 \mathrm{~A}$ & $116.1(2)$ & $\mathrm{C} 24 \mathrm{~B}-\mathrm{C} 23 \mathrm{~B}-\mathrm{C} 22 \mathrm{~B}$ & $116.1(3)$ \\
\hline $\mathrm{C} 20 \mathrm{~A}-\mathrm{C} 19 \mathrm{~A}-\mathrm{C} 8 \mathrm{~A}$ & $119.2(2)$ & $\mathrm{C} 24 \mathrm{~B}-\mathrm{C} 23 \mathrm{~B}-\mathrm{C} 27 \mathrm{~B}$ & $108.0(10)$ \\
\hline $\mathrm{C} 4 \mathrm{~B}-\mathrm{C} 9 \mathrm{~B}-\mathrm{C} 8 \mathrm{~B}$ & $108.8(2)$ & $\mathrm{C} 24 \mathrm{~B}-\mathrm{C} 23 \mathrm{~B}-\mathrm{C} 27 \mathrm{C}$ & $118.1(13)$ \\
\hline
\end{tabular}




\begin{tabular}{|c|c|c|c|}
\hline $\mathrm{C} 10 \mathrm{~B}-\mathrm{C} 9 \mathrm{~B}-\mathrm{C} 4 \mathrm{~B}$ & $108.6(2)$ & $\mathrm{C} 24 \mathrm{~B}-\mathrm{C} 23 \mathrm{~B}-\mathrm{C} 27 \mathrm{D}$ & $95.6(11)$ \\
\hline $\mathrm{C} 10 \mathrm{~B}-\mathrm{C} 9 \mathrm{~B}-\mathrm{C} 8 \mathrm{~B}$ & $109.5(2)$ & $\mathrm{C} 24 \mathrm{~A}-\mathrm{C} 23 \mathrm{~A}-\mathrm{C} 22 \mathrm{~A}$ & $113.9(3)$ \\
\hline $\mathrm{C} 11 \mathrm{~B}-\mathrm{C} 9 \mathrm{~B}-\mathrm{C} 4 \mathrm{~B}$ & $109.5(3)$ & $\mathrm{C} 24 \mathrm{~A}-\mathrm{C} 23 \mathrm{~A}-\mathrm{C} 27 \mathrm{~A}$ & $110.7(3)$ \\
\hline $\mathrm{C} 11 \mathrm{~B}-\mathrm{C} 9 \mathrm{~B}-\mathrm{C} 8 \mathrm{~B}$ & $110.6(2)$ & $\mathrm{C} 22 \mathrm{~A}-\mathrm{C} 23 \mathrm{~A}-\mathrm{C} 27 \mathrm{~A}$ & $110.4(3)$ \\
\hline $\mathrm{C} 11 \mathrm{~B}-\mathrm{C} 9 \mathrm{~B}-\mathrm{C} 10 \mathrm{~B}$ & $109.8(3)$ & $\mathrm{C} 1 \mathrm{~N}-\mathrm{C} 6 \mathrm{~N}-\mathrm{C} 5 \mathrm{~N}$ & $111.5(3)$ \\
\hline $\mathrm{C} 15 \mathrm{~B}-\mathrm{C} 14 \mathrm{~B}-\mathrm{C} 13 \mathrm{~B}$ & $120.8(3)$ & $\mathrm{C} 23 \mathrm{~A}-\mathrm{C} 24 \mathrm{~A}-\mathrm{C} 25 \mathrm{~A}$ & $118.2(3)$ \\
\hline $\mathrm{O} 4 \mathrm{~A}-\mathrm{C} 21 \mathrm{~A}-\mathrm{C} 20 \mathrm{~A}$ & $124.2(3)$ & $\mathrm{C} 26 \mathrm{~A}-\mathrm{C} 24 \mathrm{~A}-\mathrm{C} 23 \mathrm{~A}$ & $119.4(3)$ \\
\hline $\mathrm{O} 4 \mathrm{~A}-\mathrm{C} 21 \mathrm{~A}-\mathrm{C} 6 \mathrm{~A}$ & $116.3(2)$ & $\mathrm{C} 26 \mathrm{~A}-\mathrm{C} 24 \mathrm{~A}-\mathrm{C} 25 \mathrm{~A}$ & $122.4(4)$ \\
\hline $\mathrm{C} 20 \mathrm{~A}-\mathrm{C} 21 \mathrm{~A}-\mathrm{C} 6 \mathrm{~A}$ & $119.4(2)$ & $\mathrm{C} 5 \mathrm{~A}-\mathrm{C} 4 \mathrm{~A}-\mathrm{C} 9 \mathrm{~A}$ & $113.0(3)$ \\
\hline $\mathrm{C} 7 \mathrm{~A}-\mathrm{C} 8 \mathrm{~A}-\mathrm{C} 19 \mathrm{~A}$ & $111.5(2)$ & $\mathrm{C} 5 \mathrm{~A}-\mathrm{C} 4 \mathrm{~A}-\mathrm{C} 3 \mathrm{~A}$ & $109.2(3)$ \\
\hline $\mathrm{C} 7 \mathrm{~A}-\mathrm{C} 8 \mathrm{~A}-\mathrm{C} 12 \mathrm{~A}$ & $109.7(2)$ & $\mathrm{C} 3 \mathrm{~A}-\mathrm{C} 4 \mathrm{~A}-\mathrm{C} 9 \mathrm{~A}$ & $113.8(3)$ \\
\hline $\mathrm{C} 7 \mathrm{~A}-\mathrm{C} 8 \mathrm{~A}-\mathrm{C} 9 \mathrm{~A}$ & $104.3(2)$ & $\mathrm{C} 17 \mathrm{~A}-\mathrm{C} 16 \mathrm{~A}-\mathrm{C} 15 \mathrm{~A}$ & $119.8(3)$ \\
\hline $\mathrm{C} 19 \mathrm{~A}-\mathrm{C} 8 \mathrm{~A}-\mathrm{C} 12 \mathrm{~A}$ & $106.0(2)$ & $\mathrm{C} 10 \mathrm{M}-\mathrm{C} 9 \mathrm{M}-\mathrm{C} 8 \mathrm{M}$ & $111.2(3)$ \\
\hline $\mathrm{C} 19 \mathrm{~A}-\mathrm{C} 8 \mathrm{~A}-\mathrm{C} 9 \mathrm{~A}$ & $110.9(2)$ & $\mathrm{C} 2 \mathrm{~N}-\mathrm{C} 1 \mathrm{~N}-\mathrm{C} 6 \mathrm{~N}$ & $110.3(3)$ \\
\hline $\mathrm{C} 12 \mathrm{~A}-\mathrm{C} 8 \mathrm{~A}-\mathrm{C} 9 \mathrm{~A}$ & $114.6(2)$ & $\mathrm{C} 6 \mathrm{~A}-\mathrm{C} 22 \mathrm{~A}-\mathrm{C} 23 \mathrm{~A}$ & 116.7 (3) \\
\hline $\mathrm{C} 16 \mathrm{~A}-\mathrm{C} 15 \mathrm{~A}-\mathrm{C} 14 \mathrm{~A}$ & $120.4(3)$ & $\mathrm{C} 25 \mathrm{~B}-\mathrm{C} 24 \mathrm{~B}-\mathrm{C} 23 \mathrm{~B}$ & $118.4(3)$ \\
\hline $\mathrm{C} 6 \mathrm{~B}-\mathrm{C} 22 \mathrm{~B}-\mathrm{C} 23 \mathrm{~B}$ & $116.2(2)$ & $\mathrm{C} 26 \mathrm{~B}-\mathrm{C} 24 \mathrm{~B}-\mathrm{C} 23 \mathrm{~B}$ & $121.6(4)$ \\
\hline $\mathrm{C} 5 \mathrm{~B}-\mathrm{C} 4 \mathrm{~B}-\mathrm{C} 9 \mathrm{~B}$ & $113.2(2)$ & $\mathrm{C} 26 \mathrm{~B}-\mathrm{C} 24 \mathrm{~B}-\mathrm{C} 25 \mathrm{~B}$ & $120.0(4)$ \\
\hline $\mathrm{C} 5 \mathrm{~B}-\mathrm{C} 4 \mathrm{~B}-\mathrm{C} 3 \mathrm{~B}$ & $108.9(2)$ & $\mathrm{C} 3 \mathrm{M}-\mathrm{C} 2 \mathrm{M}-\mathrm{C} 1 \mathrm{M}$ & $112.2(3)$ \\
\hline $\mathrm{C} 3 \mathrm{~B}-\mathrm{C} 4 \mathrm{~B}-\mathrm{C} 9 \mathrm{~B}$ & $113.0(3)$ & $\mathrm{C} 11 \mathrm{M}-\mathrm{C} 10 \mathrm{M}-\mathrm{C} 9 \mathrm{M}$ & $110.5(3)$ \\
\hline $\mathrm{C} 4 \mathrm{~N}-\mathrm{C} 5 \mathrm{~N}-\mathrm{C} 6 \mathrm{~N}$ & $111.1(2)$ & $\mathrm{C} 6 \mathrm{M}-\mathrm{C} 1 \mathrm{M}-\mathrm{C} 2 \mathrm{M}$ & 110.7 (3) \\
\hline $\mathrm{O} 2 \mathrm{~B}-\mathrm{C} 12 \mathrm{~B}-\mathrm{C} 13 \mathrm{~B}$ & $118.5(3)$ & $\mathrm{C} 10 \mathrm{M}-\mathrm{C} 11 \mathrm{M}-\mathrm{C} 12 \mathrm{M}$ & $111.2(3)$ \\
\hline
\end{tabular}




\begin{tabular}{|l|l|l|l|}
\hline $\mathrm{O} 2 \mathrm{~B}-\mathrm{C} 12 \mathrm{~B}-\mathrm{C} 8 \mathrm{~B}$ & $122.0(3)$ & $\mathrm{C} 1 \mathrm{M}-\mathrm{C} 6 \mathrm{M}-\mathrm{C} 5 \mathrm{M}$ & $111.0(3)$ \\
\hline $\mathrm{C} 13 \mathrm{~B}-\mathrm{C} 12 \mathrm{~B}-\mathrm{C} 8 \mathrm{~B}$ & $119.4(2)$ & $\mathrm{C} 28 \mathrm{~B}-\mathrm{C} 27 \mathrm{~B}-\mathrm{C} 23 \mathrm{~B}$ & $108.7(11)$ \\
\hline $\mathrm{C} 7 \mathrm{~B}-\mathrm{C} 8 \mathrm{~B}-\mathrm{C} 19 \mathrm{~B}$ & $110.8(2)$ & $\mathrm{C} 29 \mathrm{~A}-\mathrm{C} 28 \mathrm{~A}-\mathrm{C} 27 \mathrm{~A}$ & $125.7(5)$ \\
\hline $\mathrm{C} 7 \mathrm{~B}-\mathrm{C} 8 \mathrm{~B}-\mathrm{C} 9 \mathrm{~B}$ & $105.9(2)$ & $\mathrm{C} 2 \mathrm{~A}-\mathrm{C} 3 \mathrm{~A}-\mathrm{C} 4 \mathrm{~A}$ & $116.9(3)$ \\
\hline $\mathrm{C} 7 \mathrm{~B}-\mathrm{C} 8 \mathrm{~B}-\mathrm{C} 12 \mathrm{~B}$ & $109.4(2)$ & $\mathrm{C} 28 \mathrm{~A}-\mathrm{C} 27 \mathrm{~A}-\mathrm{C} 23 \mathrm{~A}$ & $112.8(3)$ \\
\hline $\mathrm{C} 19 \mathrm{~B}-\mathrm{C} 8 \mathrm{~B}-\mathrm{C} 9 \mathrm{~B}$ & $110.1(2)$ & $\mathrm{C} 1 \mathrm{~A}-\mathrm{C} 2 \mathrm{~A}-\mathrm{C} 3 \mathrm{~A}$ & $128.8(4)$ \\
\hline $\mathrm{C} 19 \mathrm{~B}-\mathrm{C} 8 \mathrm{~B}-\mathrm{C} 12 \mathrm{~B}$ & $106.4(2)$ & $\mathrm{C} 29 \mathrm{~B}-\mathrm{C} 28 \mathrm{~B}-\mathrm{C} 27 \mathrm{~B}$ & $121.1(15)$ \\
\hline $\mathrm{C} 12 \mathrm{~B}-\mathrm{C} 8 \mathrm{~B}-\mathrm{C} 9 \mathrm{~B}$ & $114.4(2)$ & $\mathrm{C} 29 \mathrm{C}-\mathrm{C} 28 \mathrm{C}-\mathrm{C} 27 \mathrm{C}$ & $127.4(18)$ \\
\hline $\mathrm{C} 7 \mathrm{~A}-\mathrm{C} 6 \mathrm{~A}-\mathrm{C} 21 \mathrm{~A}$ & $111.9(2)$ & $\mathrm{C} 29 \mathrm{D}-\mathrm{C} 28 \mathrm{D}-\mathrm{C} 27 \mathrm{D}$ & $129(3)$ \\
\hline $\mathrm{C} 7 \mathrm{~A}-\mathrm{C} 6 \mathrm{~A}-\mathrm{C} 5 \mathrm{~A}$ & $102.5(2)$ & $\mathrm{C} 28 \mathrm{C}-\mathrm{C} 27 \mathrm{C}-\mathrm{C} 23 \mathrm{~B}$ & $113.1(13)$ \\
\hline $\mathrm{C7A}-\mathrm{C} 6 \mathrm{~A}-\mathrm{C} 22 \mathrm{~A}$ & $112.5(2)$ & $\mathrm{C} 28 \mathrm{D}-\mathrm{C} 27 \mathrm{D}-\mathrm{C} 23 \mathrm{~B}$ & $108.1(16)$ \\
\hline
\end{tabular}

\section{References}

S1 Wang, W.; Cencic, R.; Whitesell, L.; Pelletier, J.; Porco, J. A., Jr. Chem. Eur. J. 2016, 22, 12006-12010.

S2 Boyce, J. H.; Porco, J. A., Jr. Angew. Chem., Int. Ed. 2014, 53, 7832-7837.

S3 Boyce, J. H.; Eschenbrenner-Lux, V.; Porco, J. A., Jr. J. Am. Chem. Soc. 2016, 138, 14789-14797.

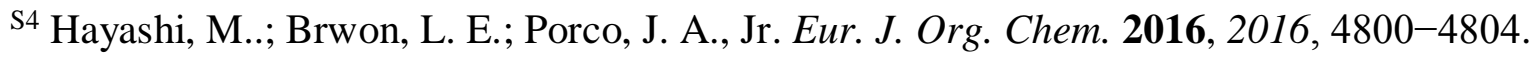

S5 Iyer, A.; Clay, A.; Jockusch, S.; Sivaguru, J. J. Phys. Org. Chem. 2017, 30, 3738-3742.

S6 Tsukano, C.; Siegel, D. R.; Danishefsky, S. J. Angew. Chem., Int. Ed. 2007, 46, 8840-8844. 
V. Select NMR Spectra 


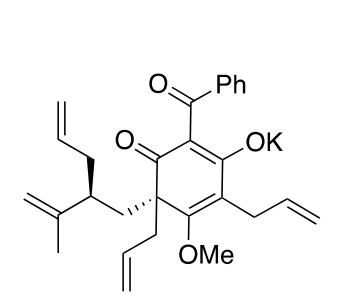

$(-)-(S, S)-5-K$

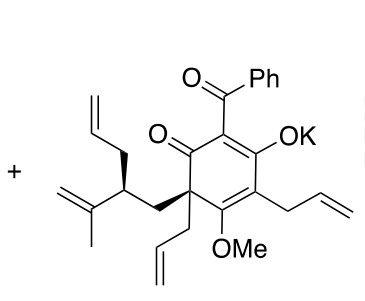

$(+)-(S, R)-5-\mathrm{K}$

ratio $=1.3: 1$

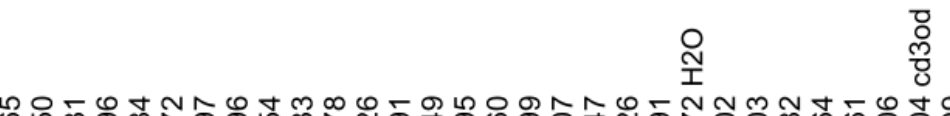

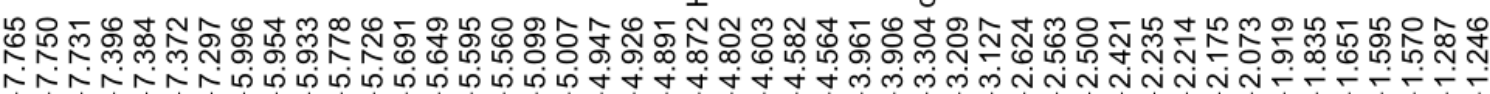

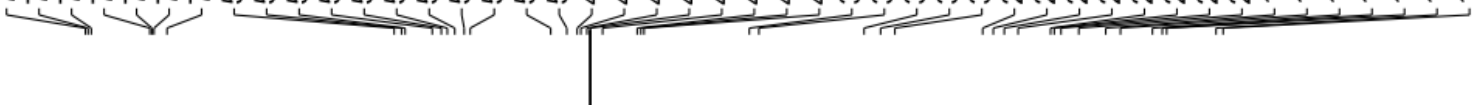

$400 \mathrm{MHz}, \mathrm{CD}_{3} \mathrm{OD}$
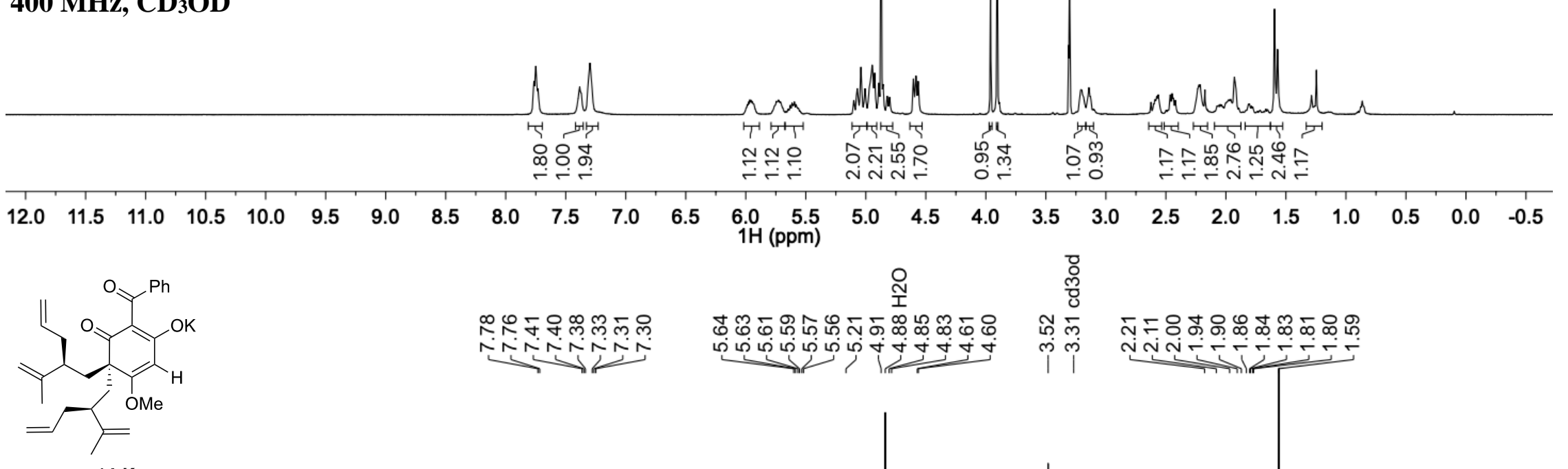

4-K

$500 \mathrm{MHz}, \mathrm{CD}_{3} \mathrm{OD}$

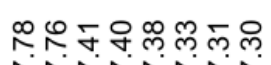
inivinising

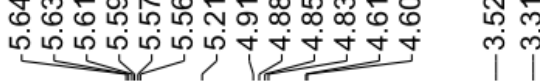

我
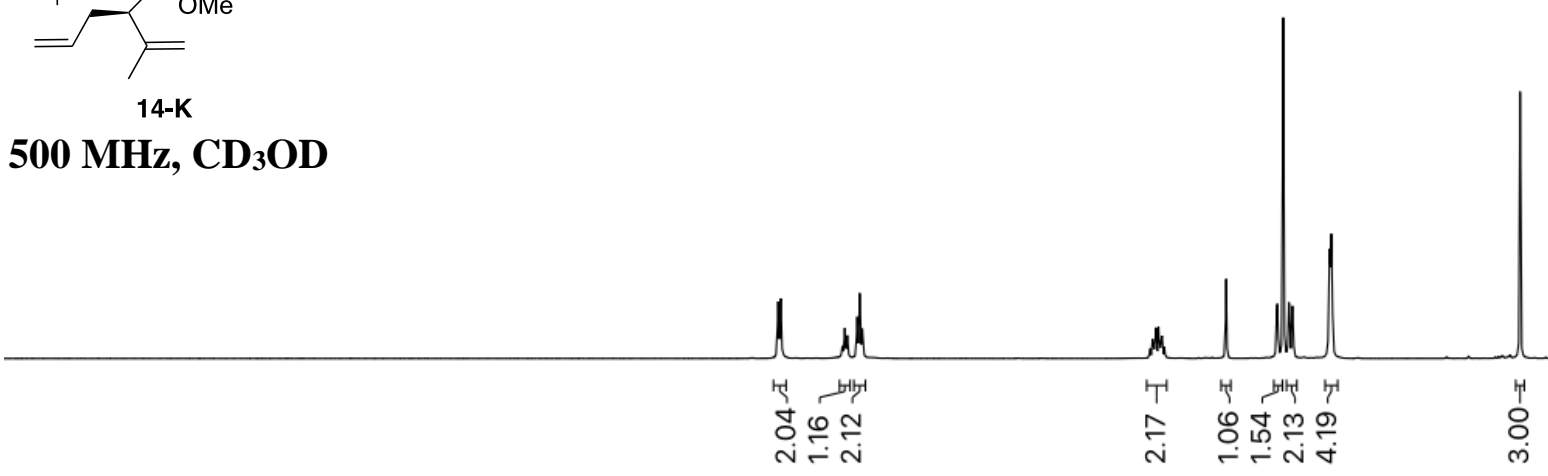

$\begin{array}{llllllllllllll}12.0 & 11.5 & 11.0 & 10.5 & 10.0 & 9.5 & 9.0 & 8.5 & 8.0 & 7.5 & 7.0 & 6.5 & 6.0 & 5.5 \\ 1 \mathrm{H}(\mathrm{ppm})\end{array}$ 

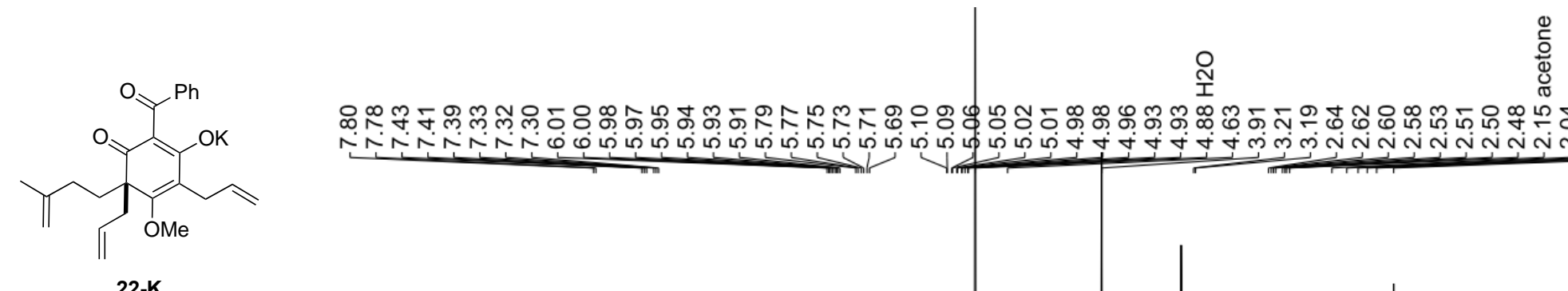

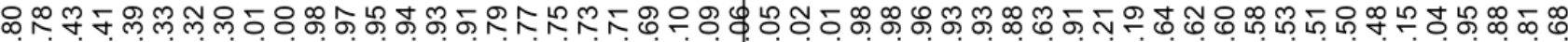

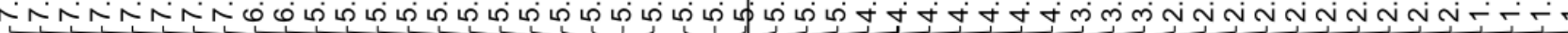

22-K

$400 \mathrm{MHz}, \mathrm{CD}_{3} \mathrm{OD}$

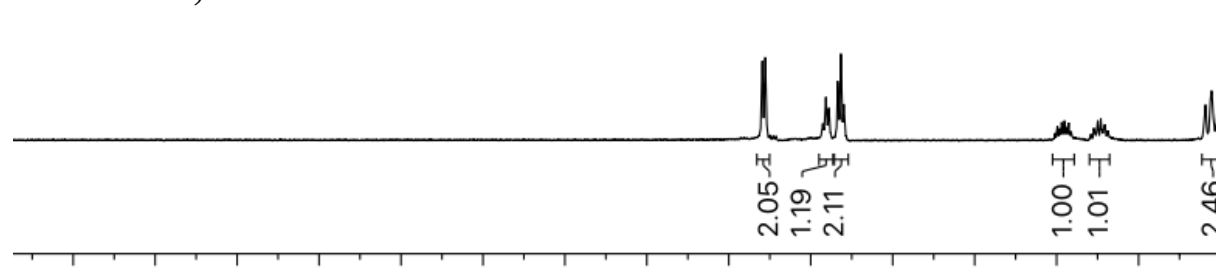

Ninil

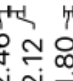

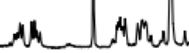

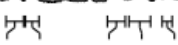

\begin{tabular}{|c|c|}
\hline Oㅇ & กิ \\
\hline
\end{tabular}

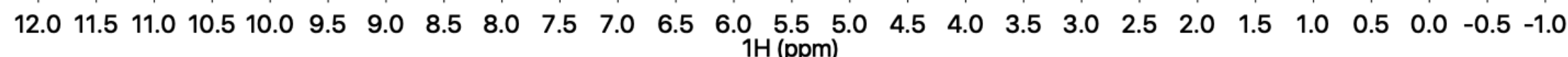

г্ণ

$101 \mathrm{MHz}, \mathrm{CD}_{3} \mathrm{OD}$

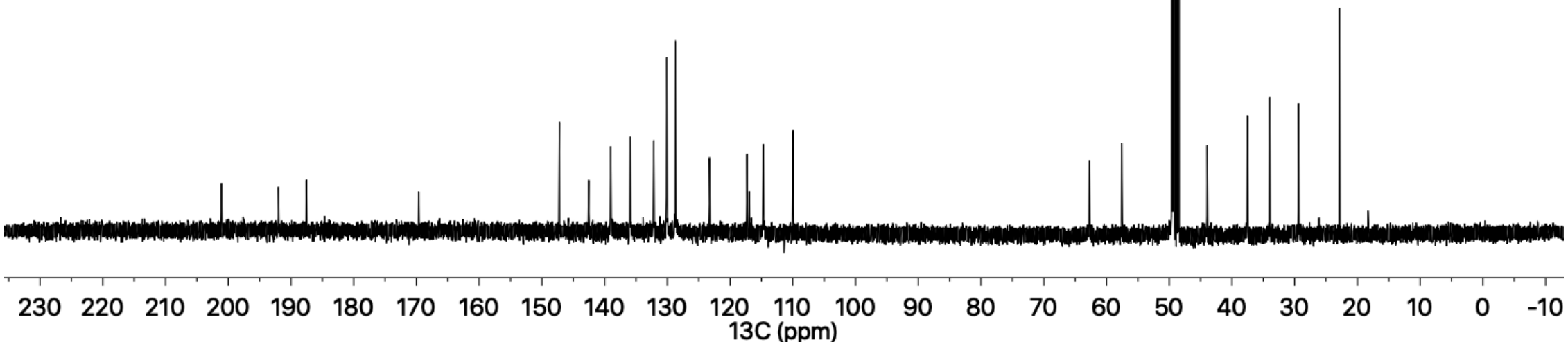




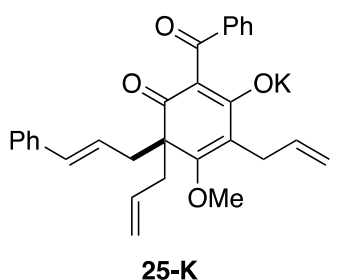

$500 \mathrm{MHz}, \mathrm{CD}_{3} \mathrm{OD}$

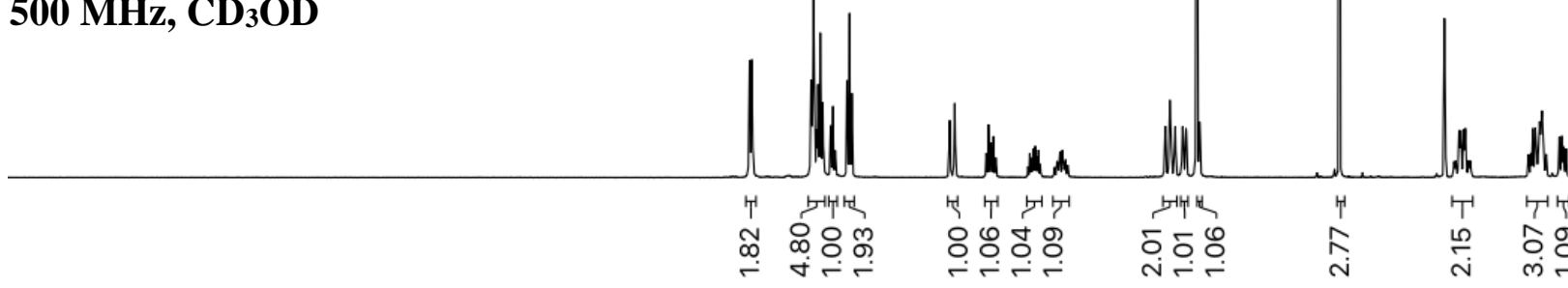

$\begin{array}{llllllllllllllllllllllllllllllllll}12.0 & 11.5 & 11.0 & 10.5 & 10.0 & 9.5 & 9.0 & 8.5 & 8.0 & 7.5 & 7.0 & 6.5 & 6.0 & 5.5 & 5.0 & 4.5 & 4.0 & 3.5 & 3.0 & 2.5 & 2.0 & 1.5 & 1.0 & 0.5 & 0.0 & -0.5 & -1.0\end{array}$

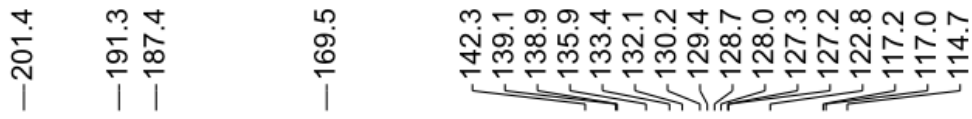

$126 \mathrm{MHz}, \mathrm{CD}_{3} \mathrm{OD}$

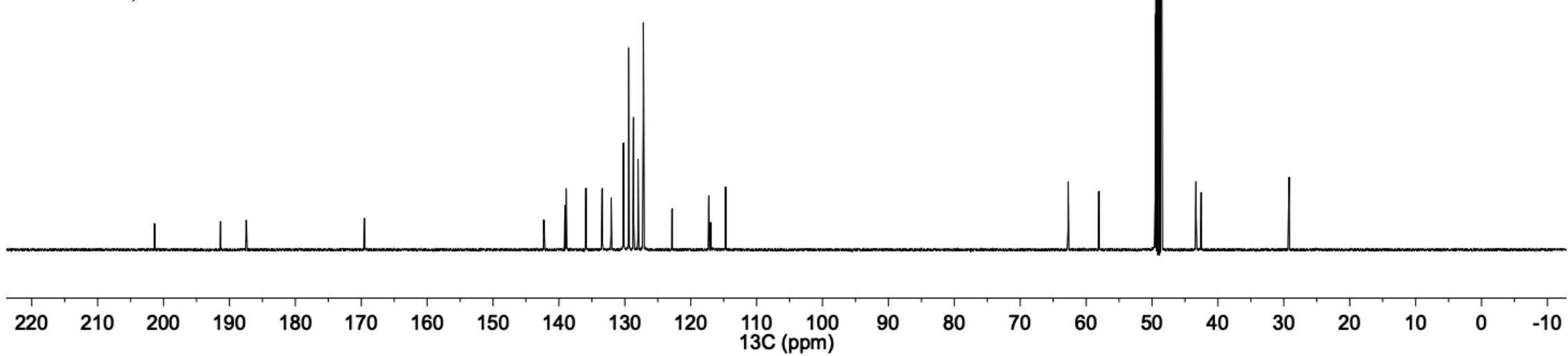




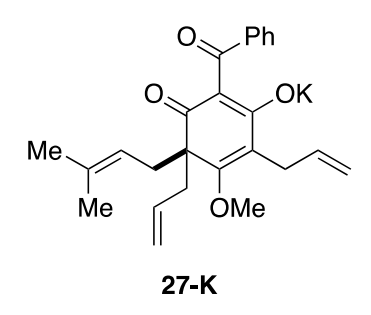

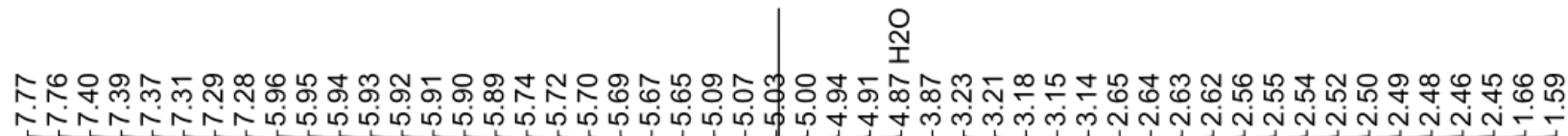

$500 \mathrm{MHz}, \mathrm{CD}_{3} \mathrm{OD}$
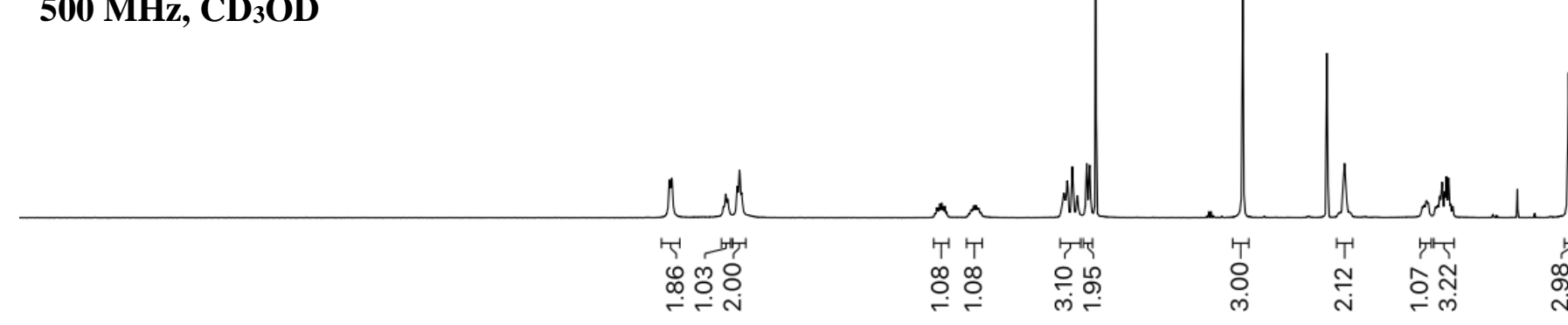

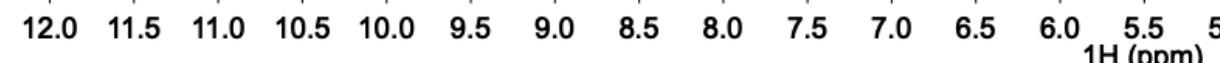

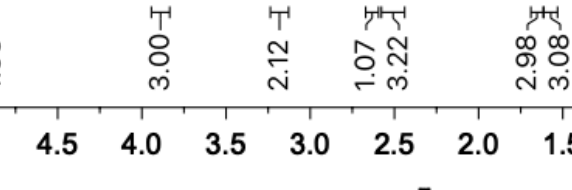

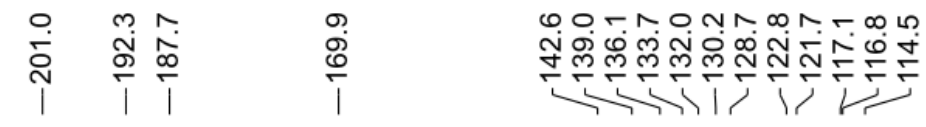
$126 \mathrm{MHz}, \mathrm{CD}_{3} \mathrm{OD}$

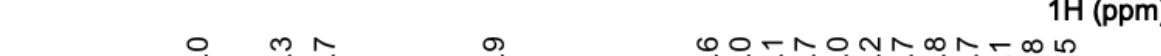
-




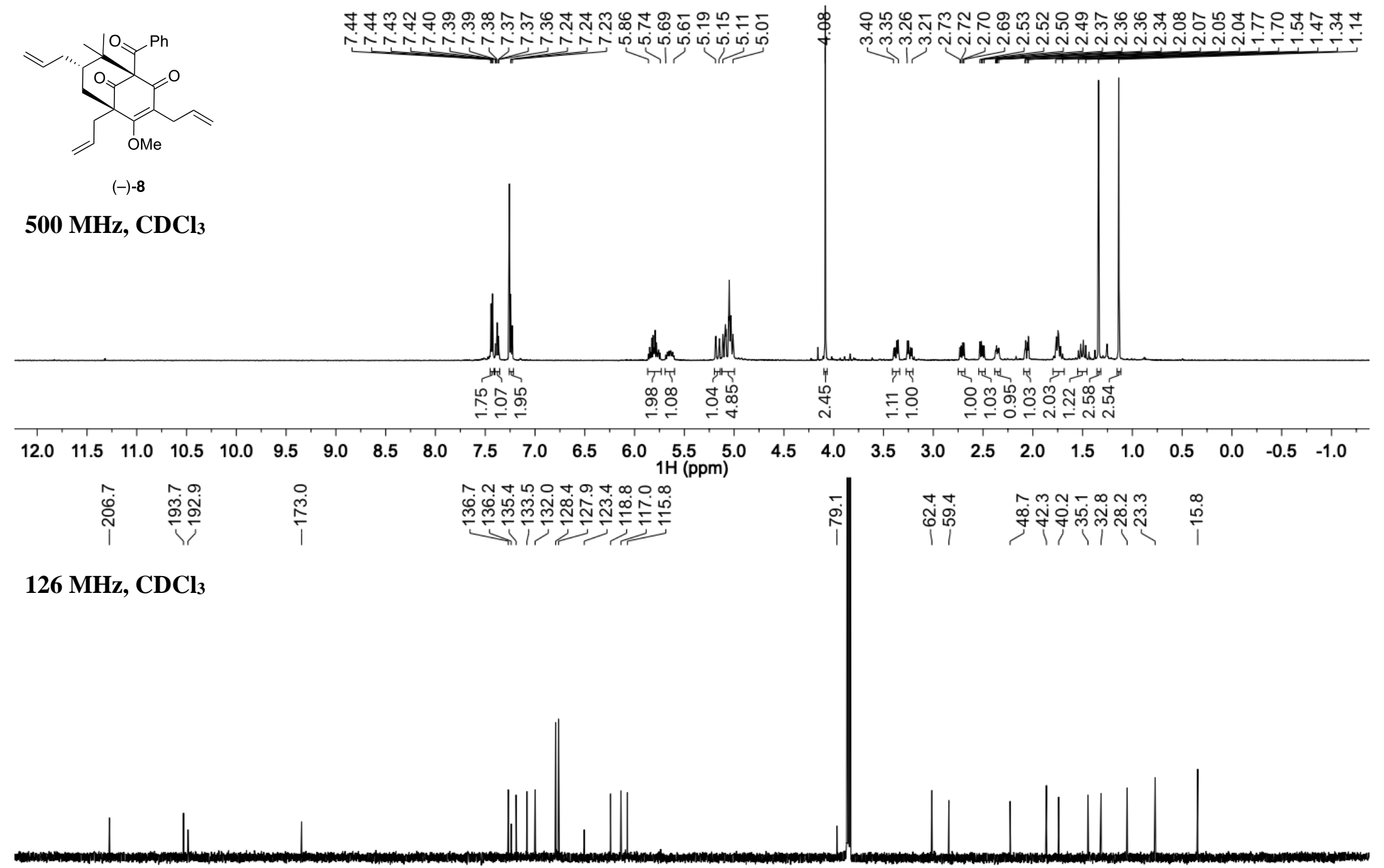

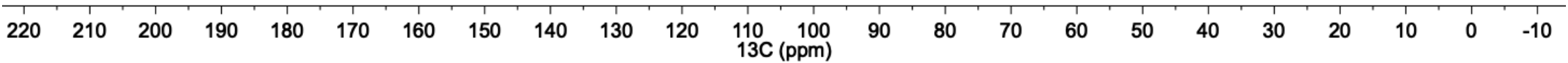


Partial NOESY Spectrum of (-)-8 (500 MHz, benzene- $d 6)$, Key ${ }^{1} \mathrm{H}-{ }^{1} \mathrm{H}$ Correlation Highlighted

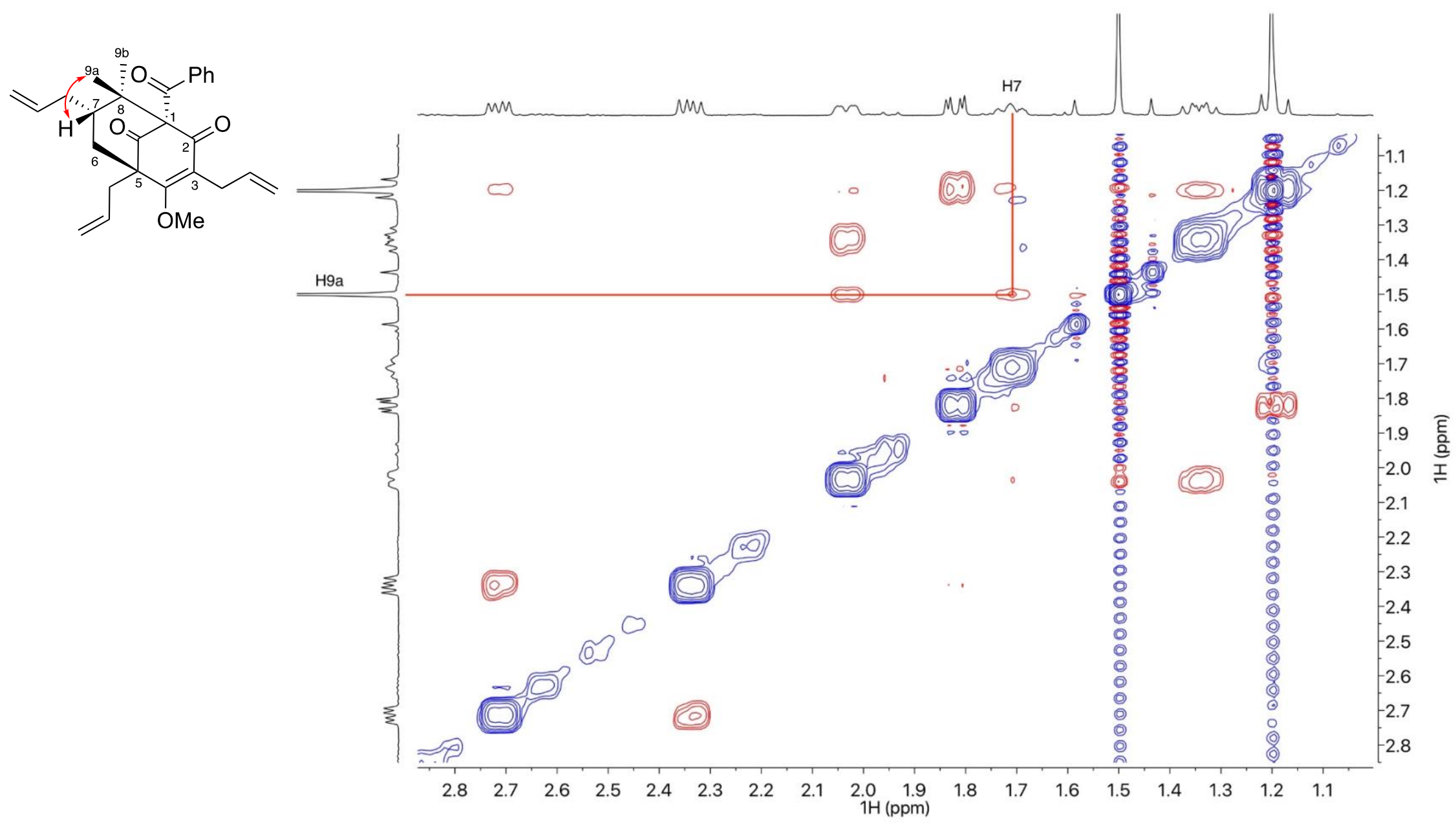




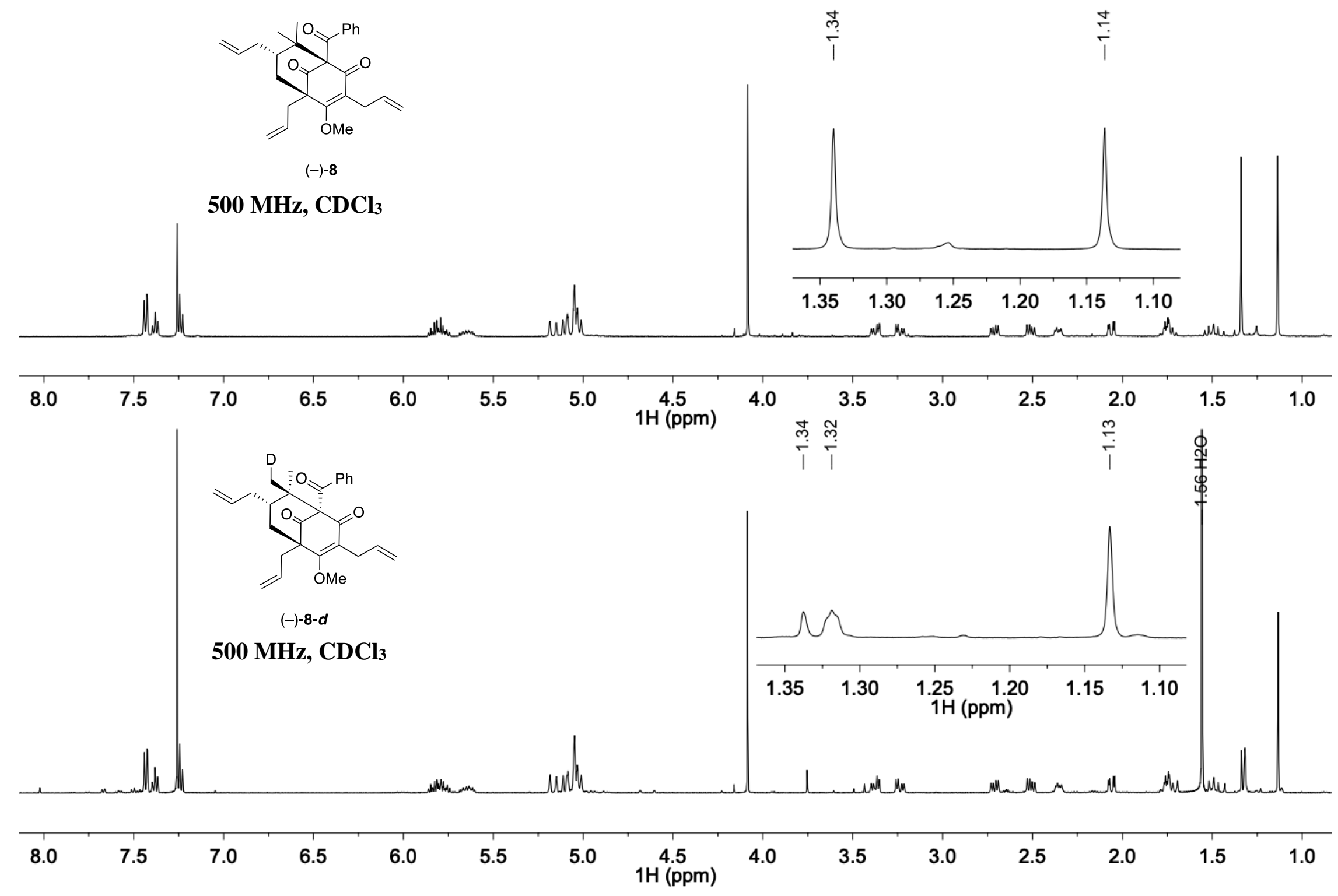




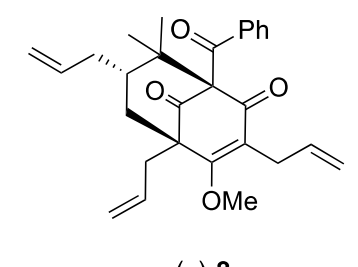

$(-)-8$

$126 \mathrm{MHz}, \mathrm{CDCl}_{3}$
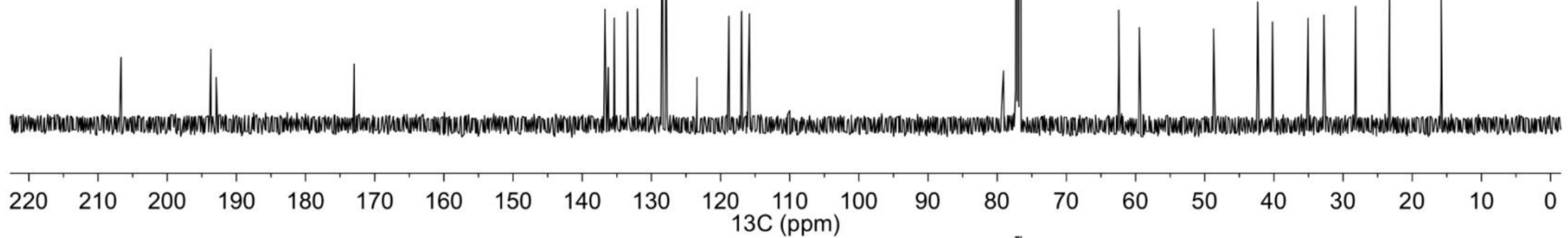

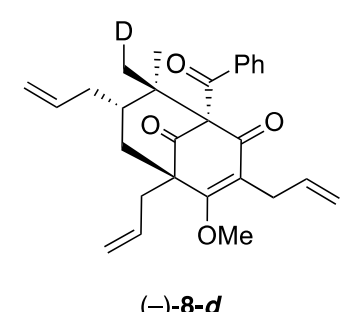

$126 \mathrm{MHz}, \mathrm{CDCl}_{3}$

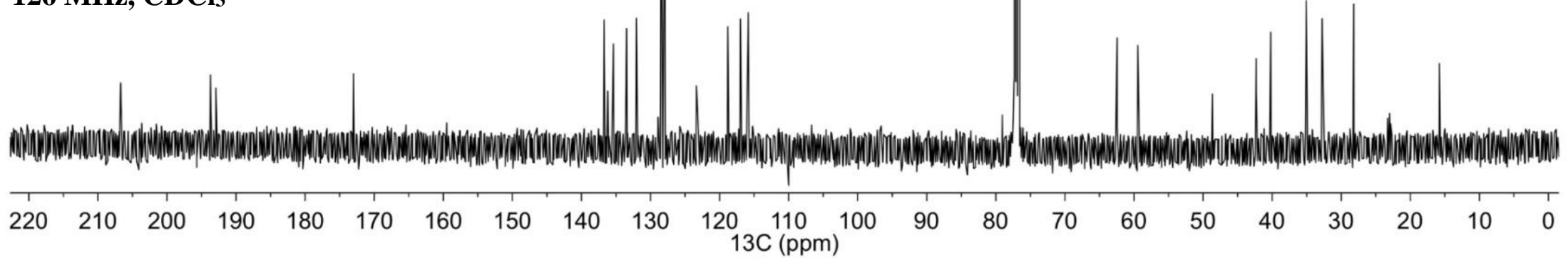


Partial gHSQC Spectrum of (-)-8-d (500 MHz), Key ${ }^{1} \mathrm{H}^{-13} \mathrm{C}$ Correlations Highlighted

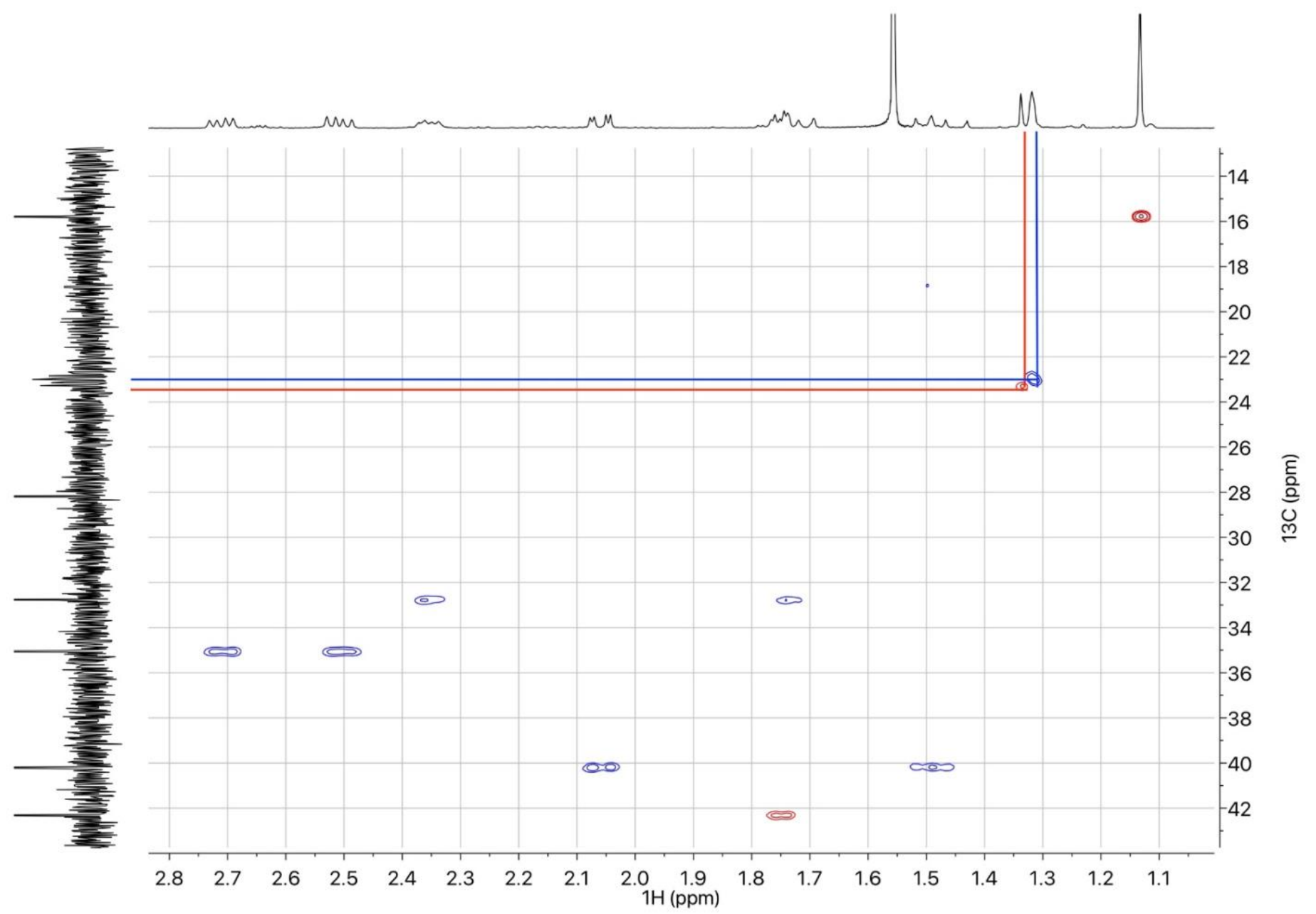




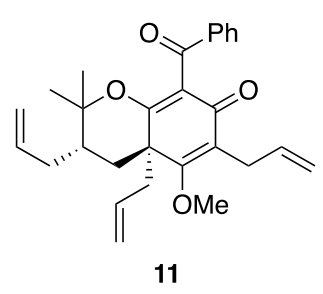

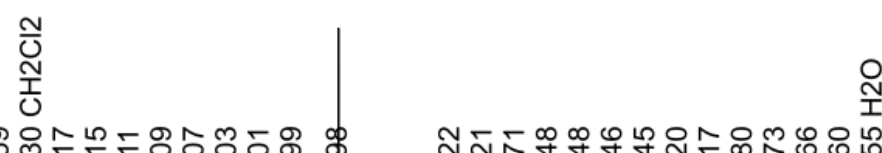
œ

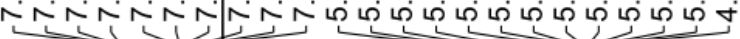

สุติ

$400 \mathrm{MHz}, \mathrm{CDCl}_{3}$

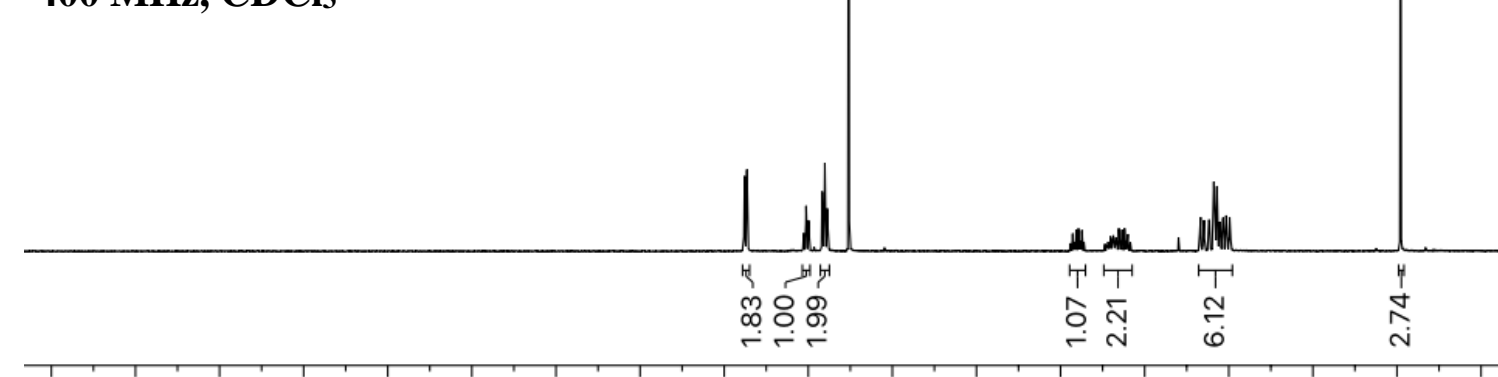

$\begin{array}{llllll}12.0 & 11.5 & 11.0 & 10.5 & 10.0 & 9.5\end{array}$

i

$\begin{array}{lllllll}8.5 & 8.0 & 7.5 & 7.0 & 6.5 & 6.0 \underset{1 H}{5}(\mathrm{ppm}) & 5.5\end{array}$

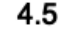

4.0

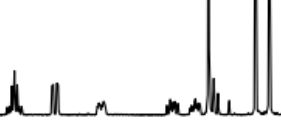

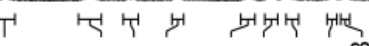

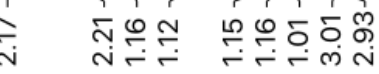

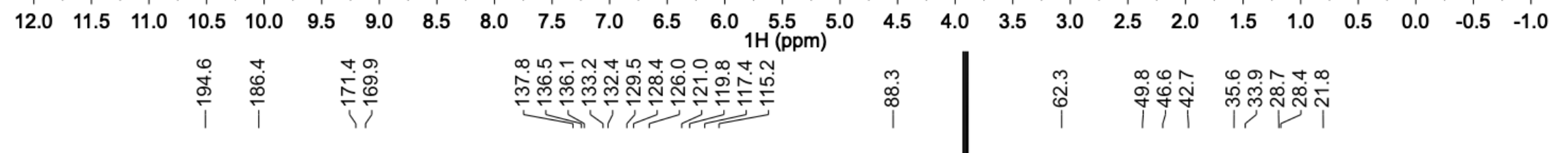

$101 \mathrm{MHz}, \mathrm{CDCl}_{3}$

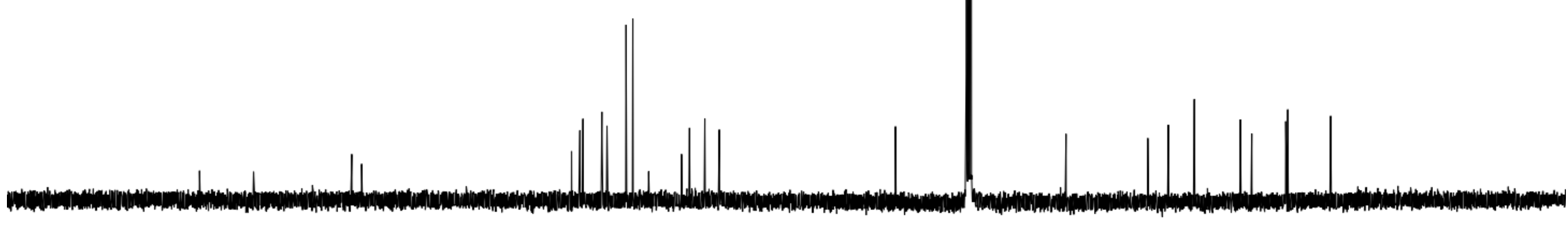

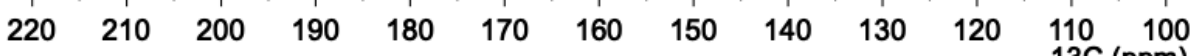

$90 \quad 80 \quad 70 \quad 60$

50

$40 \quad 30$

2010

$0-10$ 


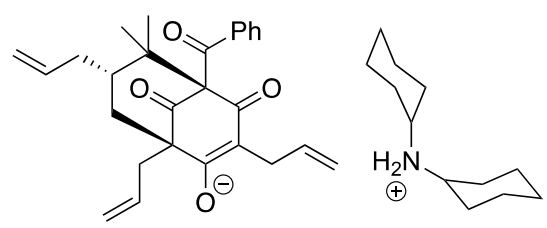

$(-)-12$

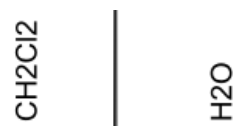

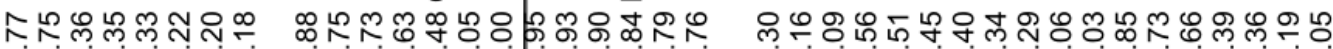
ن

$400 \mathrm{MHz}, \mathrm{CD}_{3} \mathrm{OD}$

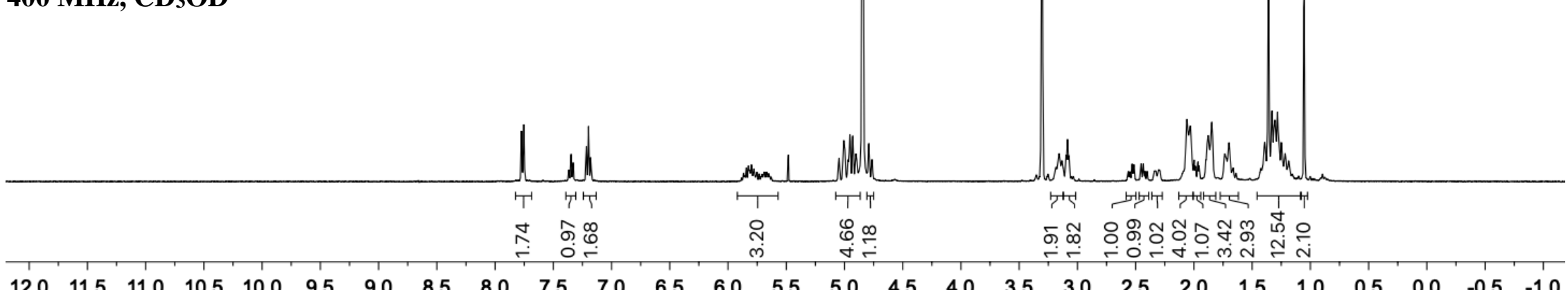

$\begin{array}{llllllllllllll}12.0 & 11.5 & 11.0 & 10.5 & 10.0 & 9.5 & 9.0 & 8.5 & 8.0 & 7.5 & 7.0 & 6.5 & 6.0 & \begin{array}{l}5.5 \\ (\mathrm{ppm})\end{array}\end{array}$

$\stackrel{m}{\stackrel{n}{i}} \quad \stackrel{m}{\infty} \frac{\infty}{i}$

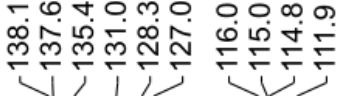

$101 \mathrm{MHz}, \mathrm{CD}_{3} \mathrm{OD}$

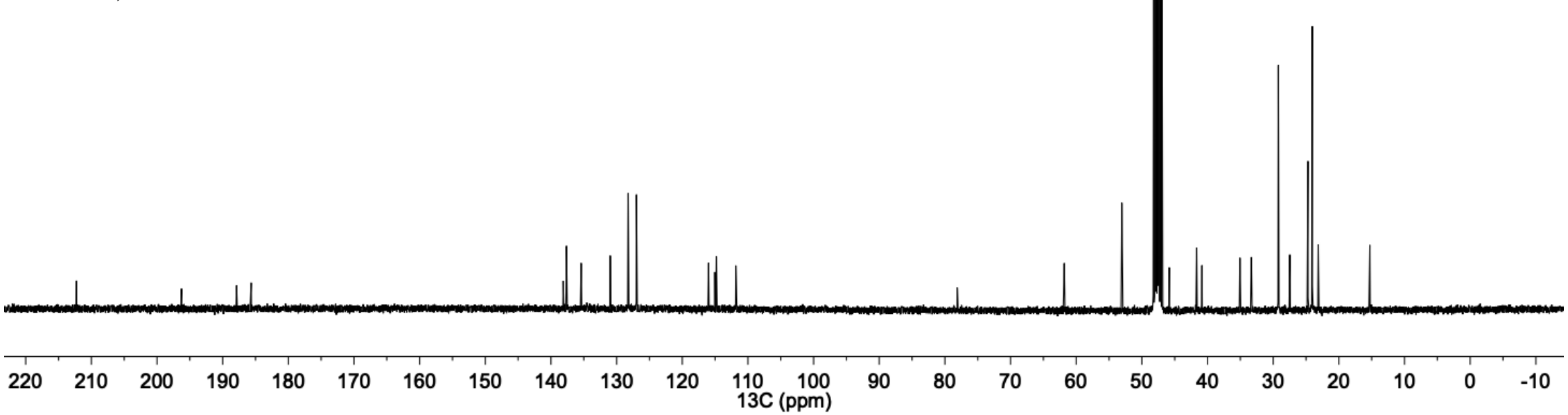




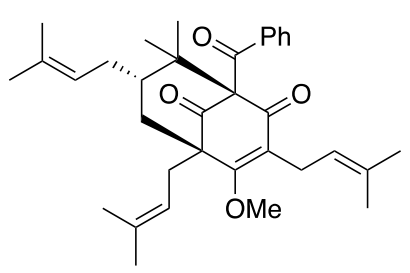

$(-)-13$

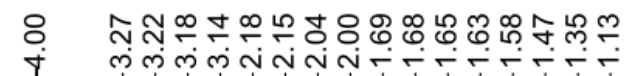

पi

$500 \mathrm{MHz}, \mathrm{CDCl}_{3}$

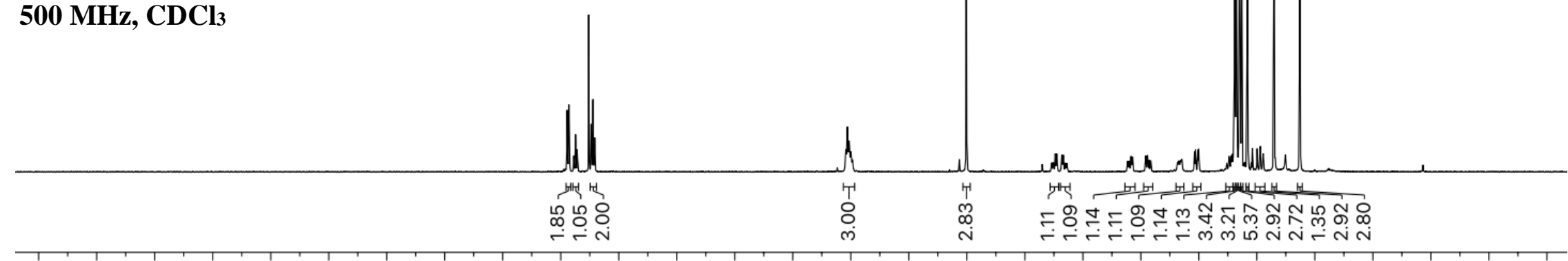

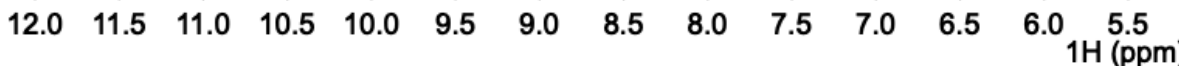

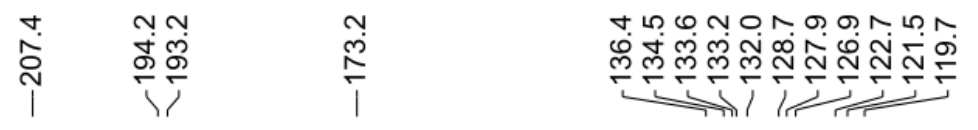

$126 \mathrm{MHz} \mathrm{CDCl}_{3}$

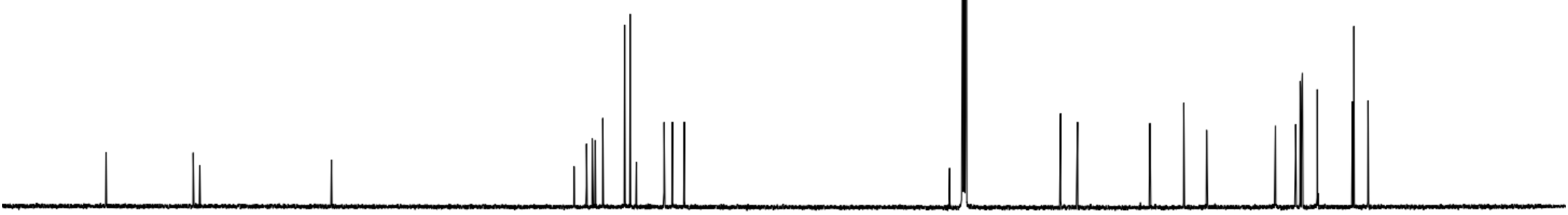

$\begin{array}{llllllllllll}220 & 210 & 200 & 190 & 180 & 170 & 160 & 150 & 140 & 130 & 120 & \begin{array}{c}110 \\ 13 \mathrm{C}(\mathrm{ppm})\end{array}\end{array}$

9080

$70 \quad 60$

50

$40 \quad 30$

20

$10 \quad 0$

$-10$ 

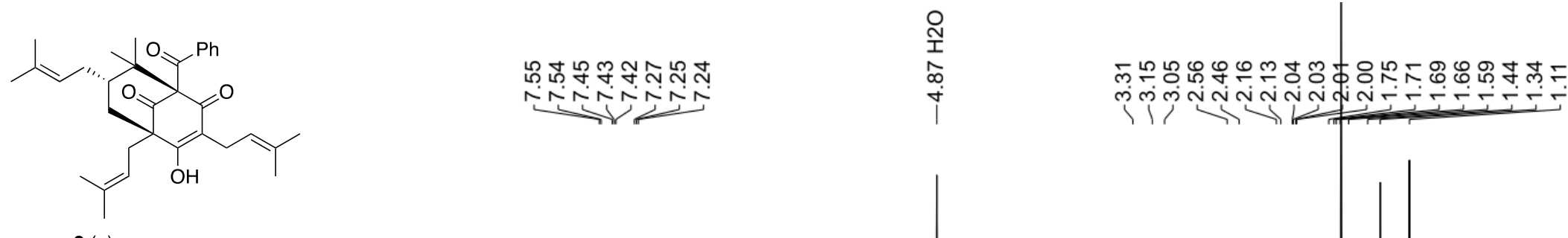

2 (-)-nemorosone $500 \mathrm{MHz}, \mathrm{CD}_{3} \mathrm{OH}$
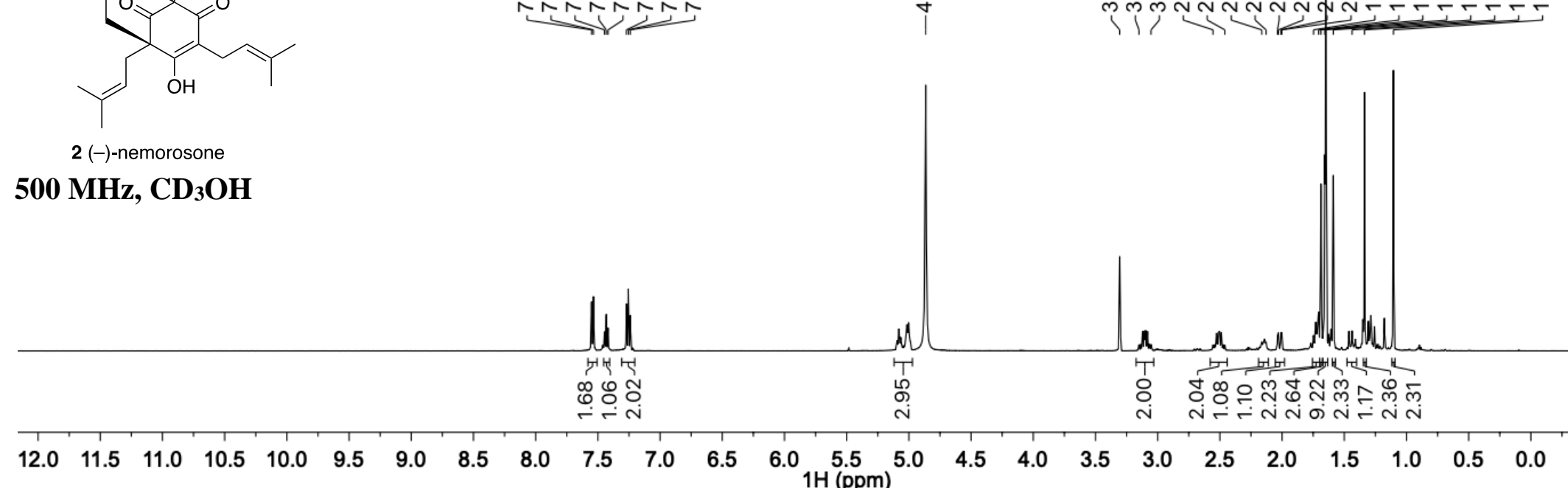

กั

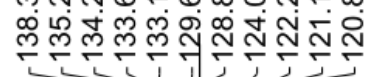

$126 \mathrm{MHz}, \mathrm{CD}_{3} \mathrm{OH}$

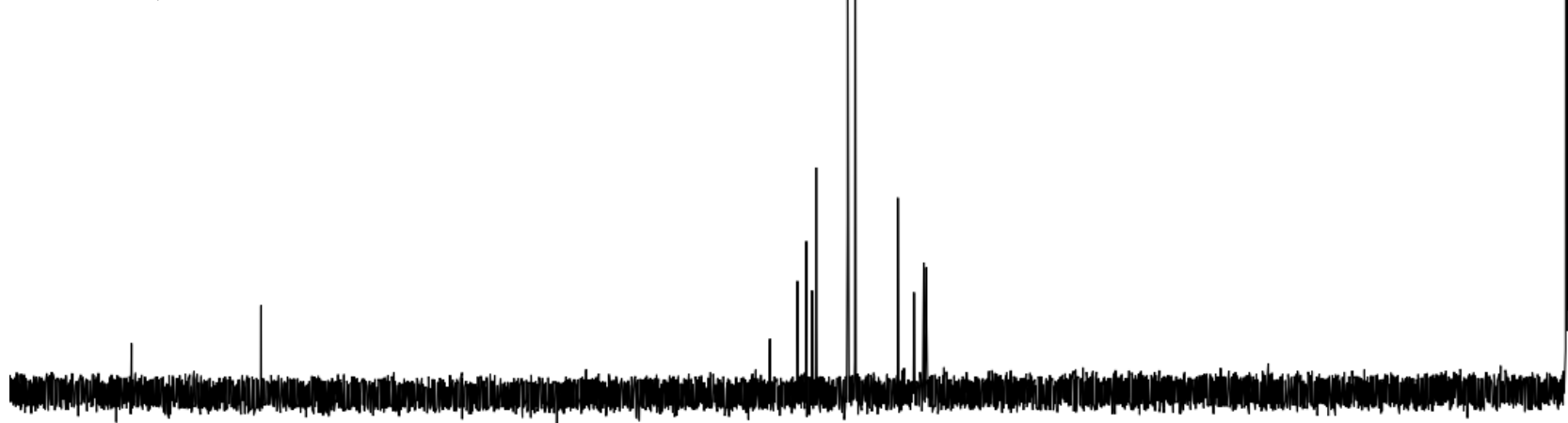

$\begin{array}{llllll}1.5 & 1.0 & 0.5 & 0.0 & -0.5 & -1.0\end{array}$

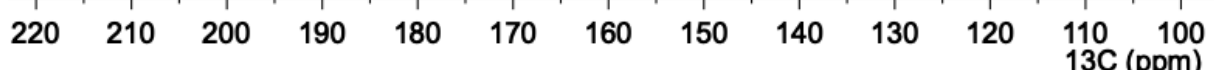

90

8070

60

$50 \quad 40$

30

$20 \quad 10$

$0 \quad-10$ 


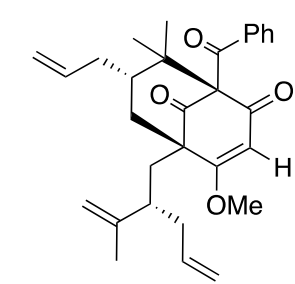

$(-)-15$

$500 \mathrm{MHz}, \mathrm{CDCl}_{3}$

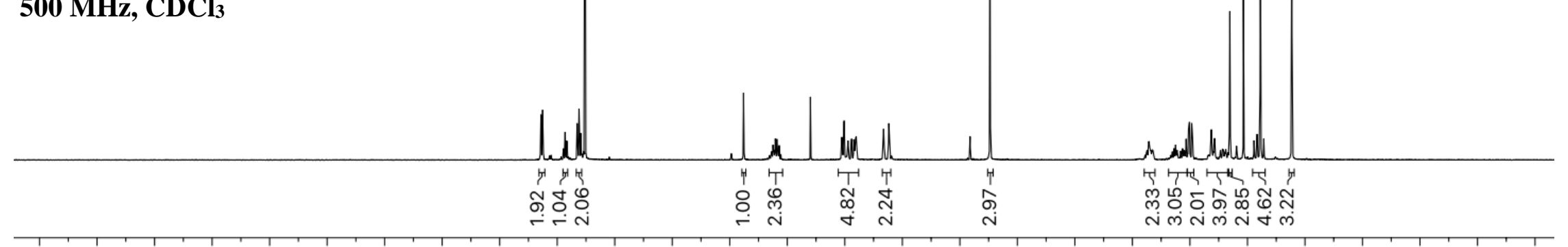

$\begin{array}{llllllllllllllllllllllllllllllllll}12.0 & 11.5 & 11.0 & 10.5 & 10.0 & 9.5 & 9.0 & 8.5 & 8.0 & 7.5 & 7.0 & 6.5 & 6.0 & \mathbf{5} .5 & 5.0 & 4.5 & 4.0 & 3.5 & 3.0 & 2.5 & 2.0 & 1.5 & 1.0 & 0.5 & 0.0 & -0.5 & -1.0\end{array}$ in

$126 \mathrm{MHz}, \mathrm{CDCl}_{3}$
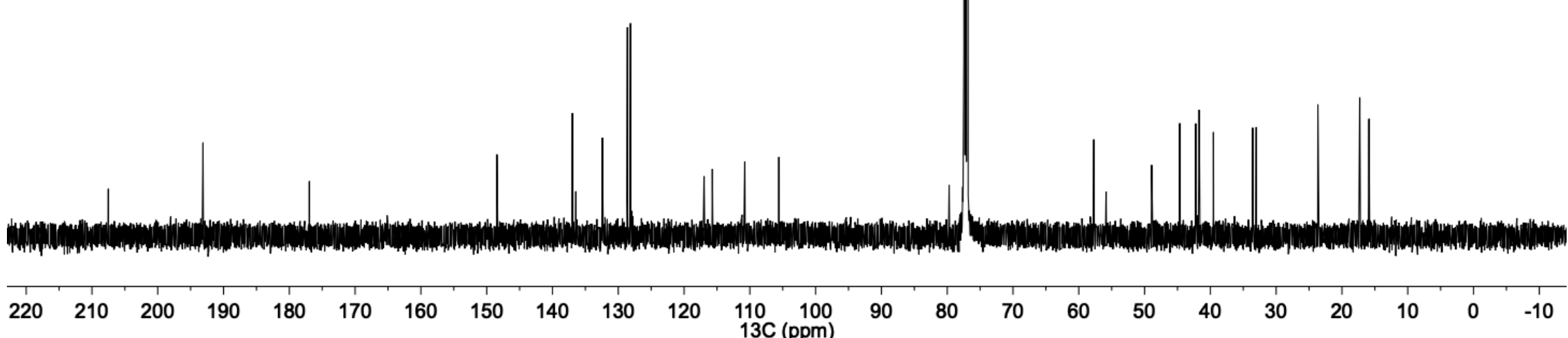


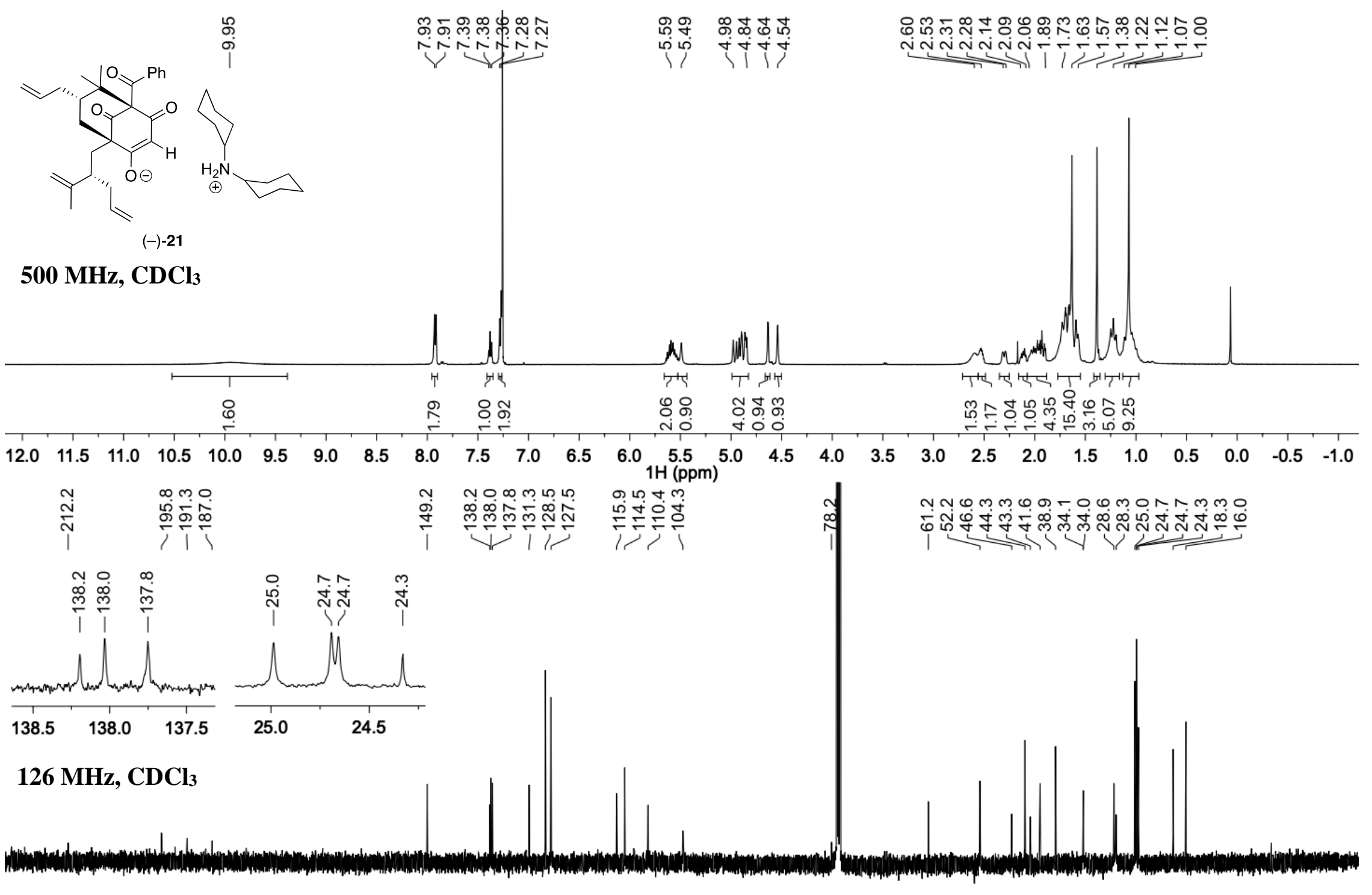

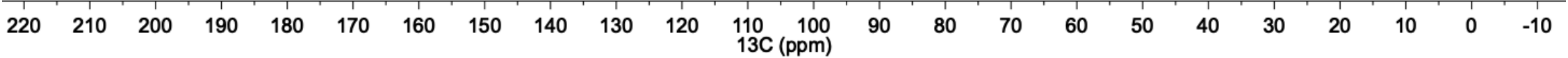




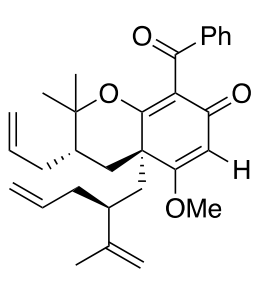

16

$500 \mathrm{MHz}, \mathrm{CDCl}_{3}$

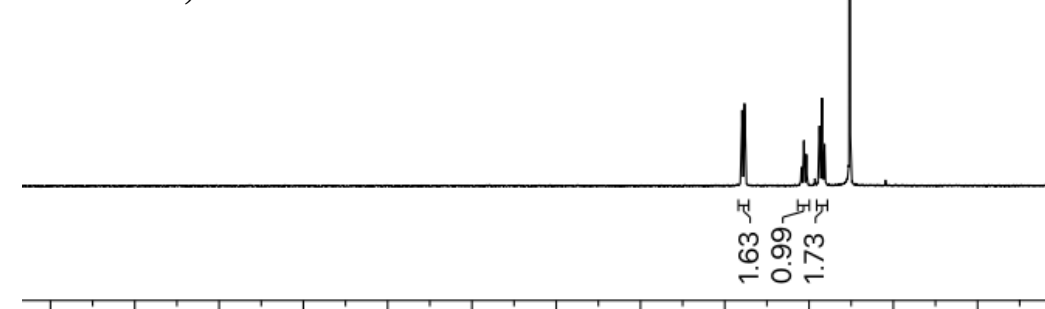

ภ요 NNNNNNNNNN

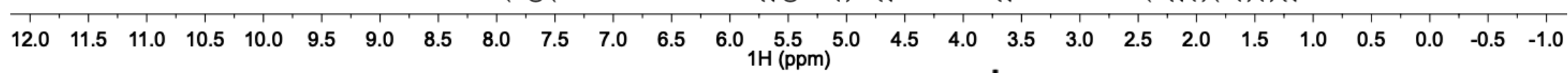

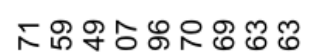

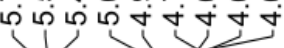

芒

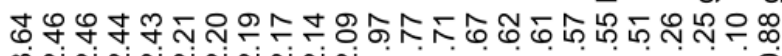
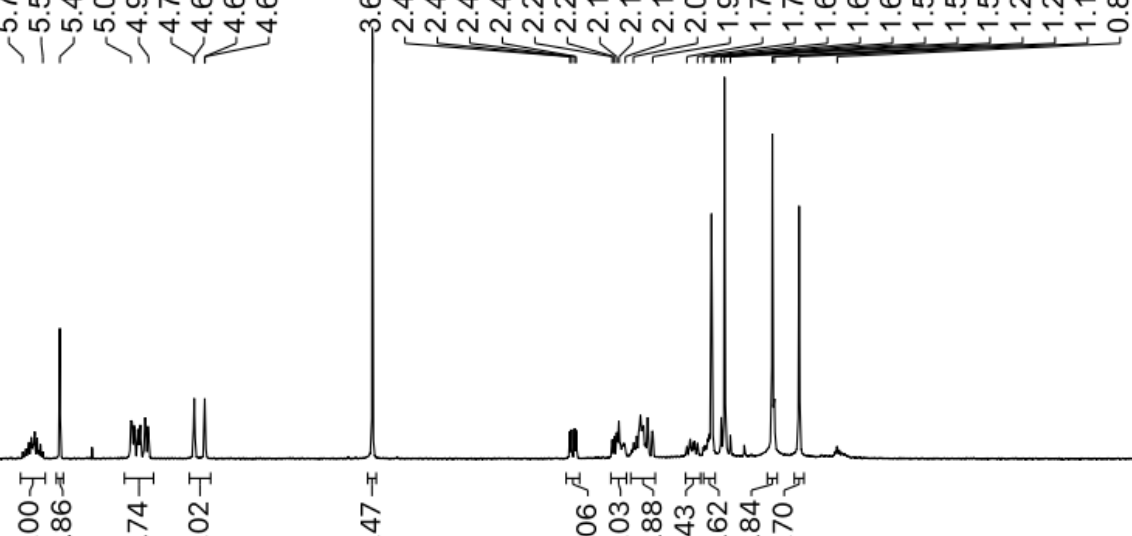
iํ ले

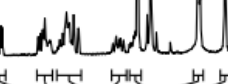

त श्ञात

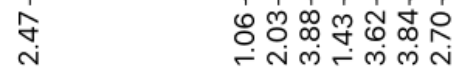
li

$126 \mathrm{MHz}, \mathrm{CDCl}_{3}$

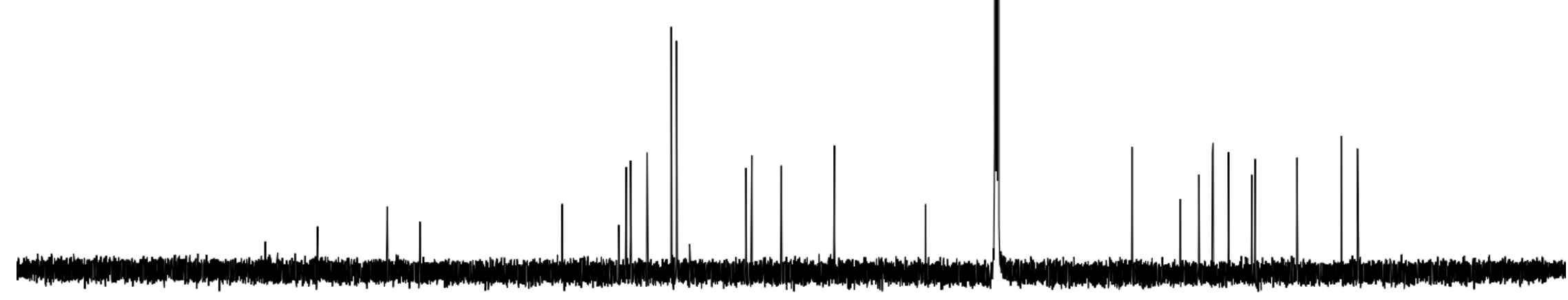

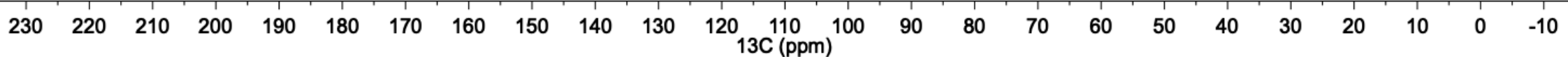




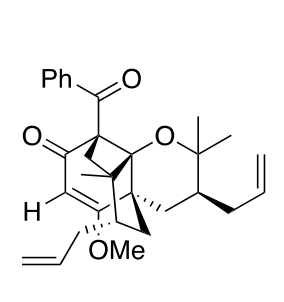

17

$500 \mathrm{MHz}, \mathrm{CDCl}_{3}$

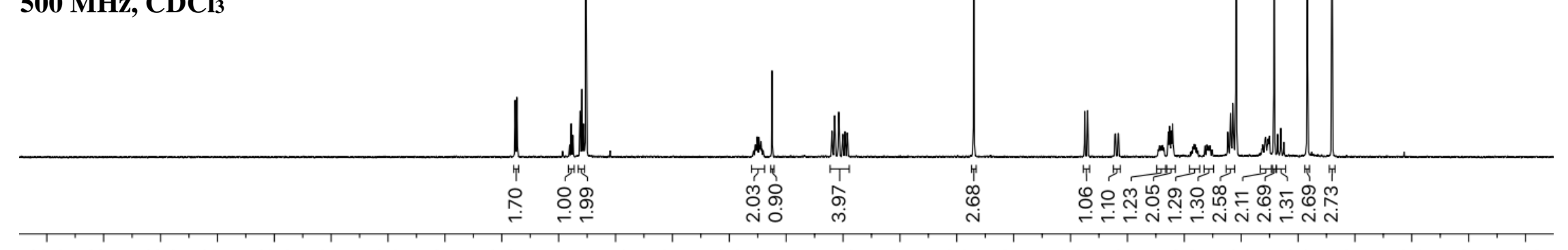

$\begin{array}{lllllllllllllllllllllllllll}12.0 & 11.5 & 11.0 & 10.5 & 10.0 & 9.5 & 9.0 & 8.5 & 8.0 & 7.5 & 7.0 & 6.5 & 6.0 & \begin{array}{l}5.5 \\ (\mathrm{ppm})\end{array} & 5.0 & 4.5 & 4.0 & 3.5 & 3.0 & 2.5 & 2.0 & 1.5 & 1.0 & 0.5 & 0.0 & -0.5 & -1.0\end{array}$

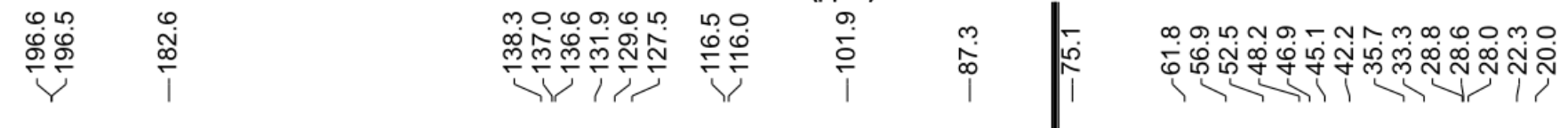

$126 \mathrm{MHz}, \mathrm{CDCl}_{3}$

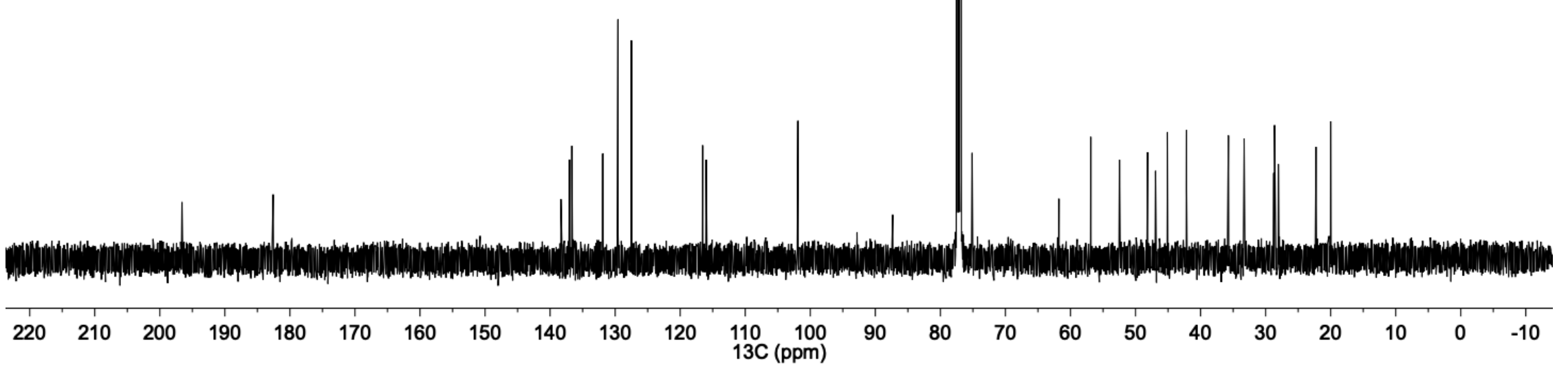


Partial gHMBC Spectrum of 17 (500/126 MHz), Key ${ }^{1} \mathrm{H}^{-13} \mathrm{C}$ Correlations Highlighted
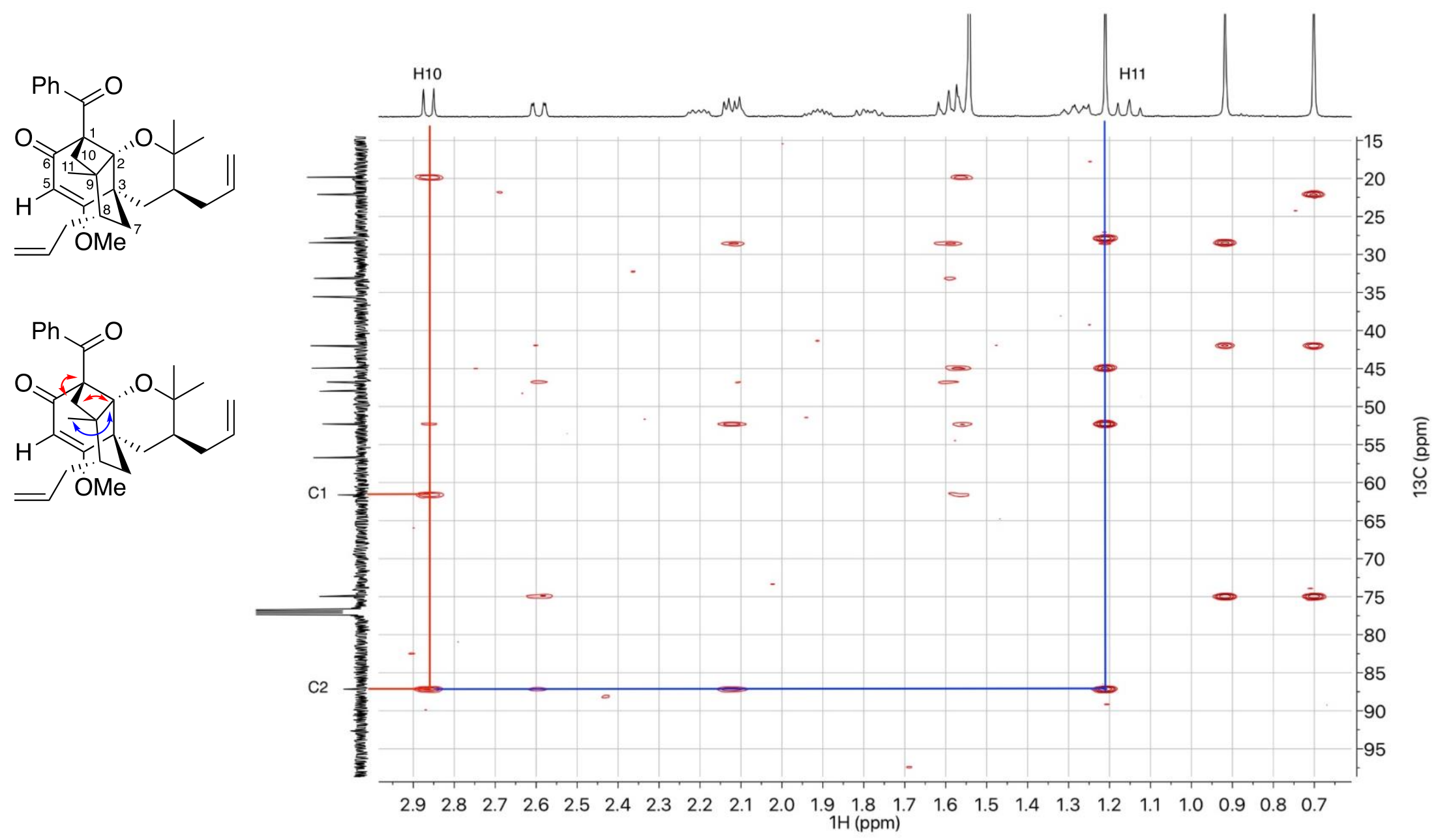
Partial NOESY Spectrum of 17 (500 MHz), Key ${ }^{1} \mathrm{H}_{-}{ }^{13} \mathrm{C}$ Correlations Highlighted

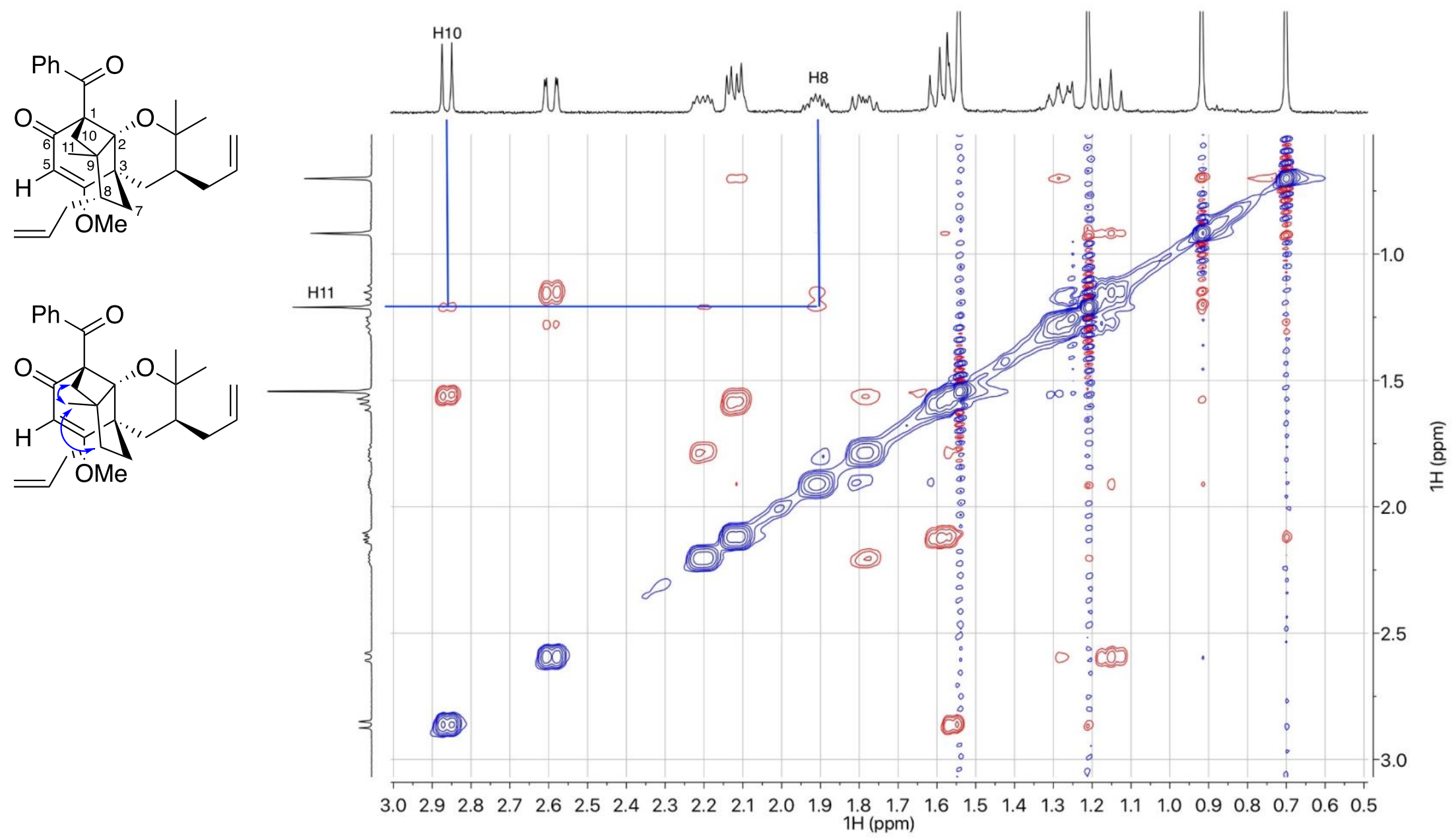




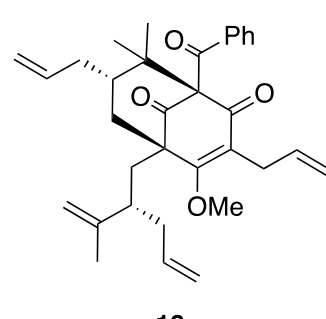

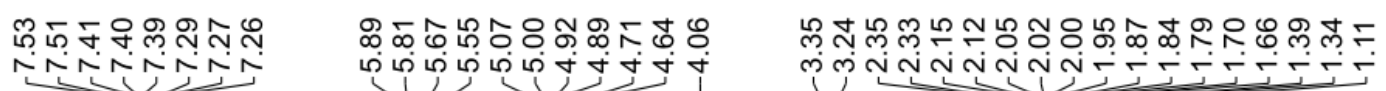

18

$500 \mathrm{MHz}^{\mathrm{CDCl}_{3}}$

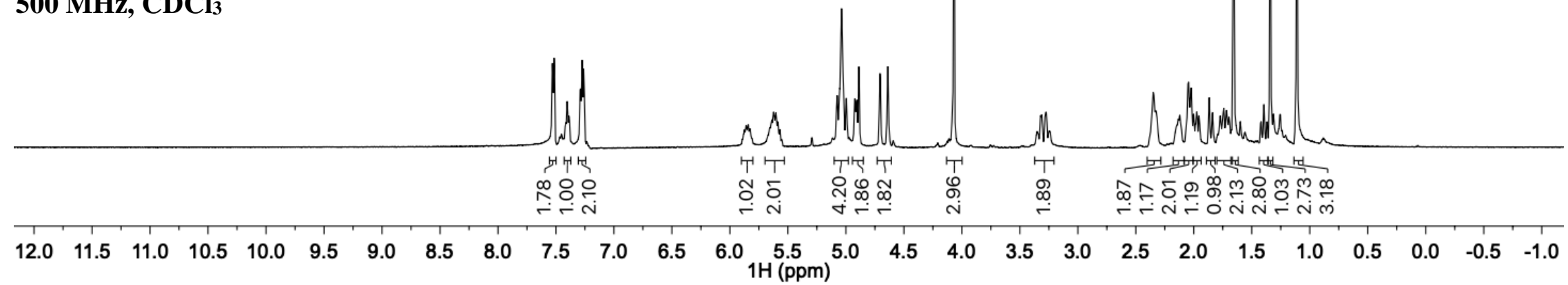
î.

$126 \mathrm{MHz}^{\mathrm{CDCl}} 3$

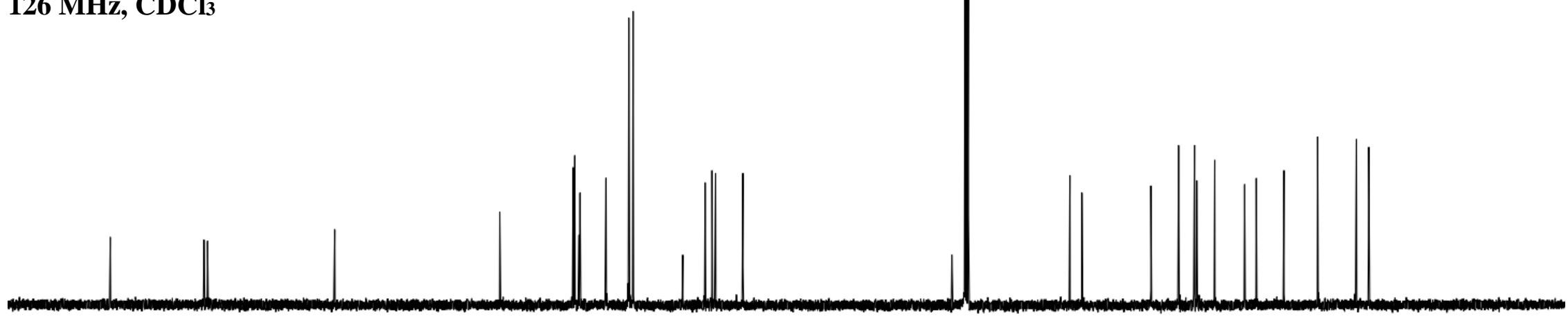

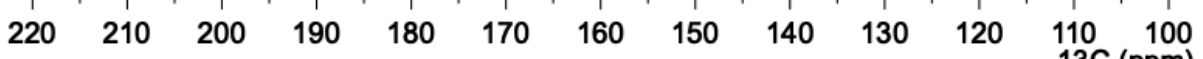
$13 \mathrm{C}(\mathrm{ppm})$

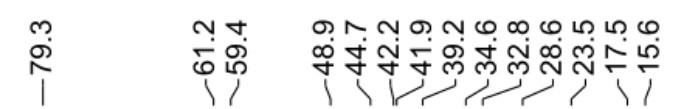

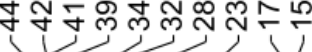




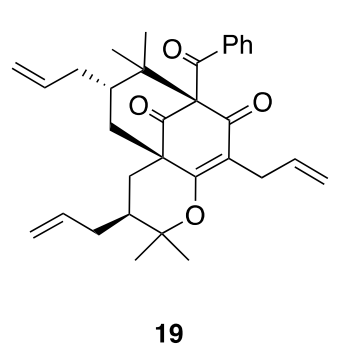

$500 \mathrm{MHz}, \mathrm{CDCl}_{3}$
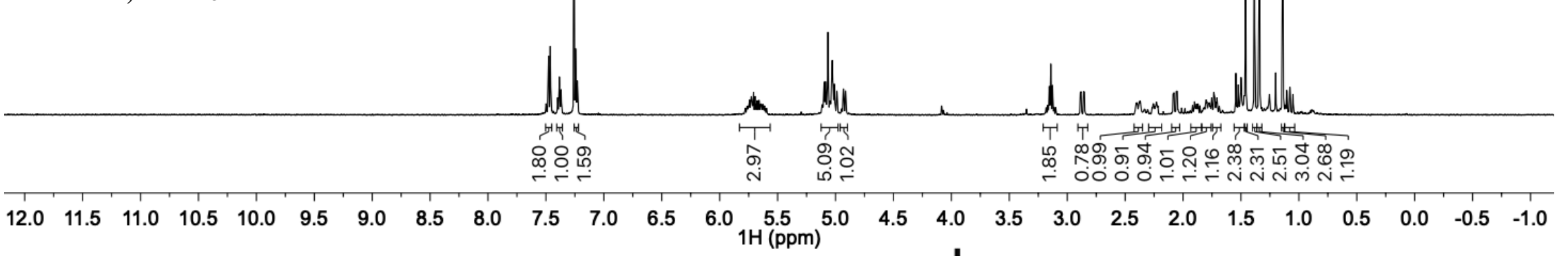
후
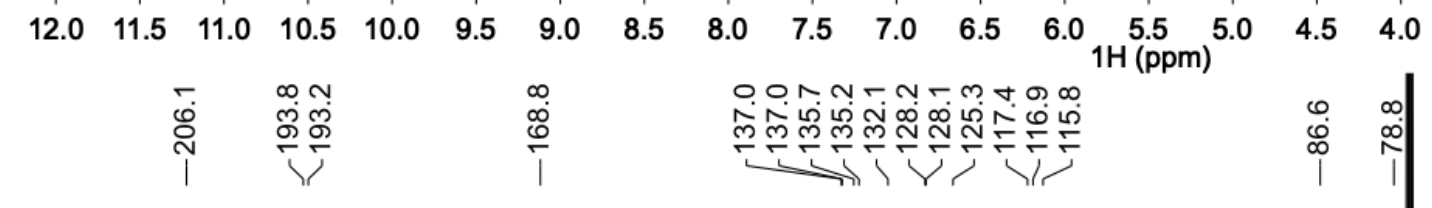

$126 \mathrm{MHz}, \mathrm{CDCl}_{3}$

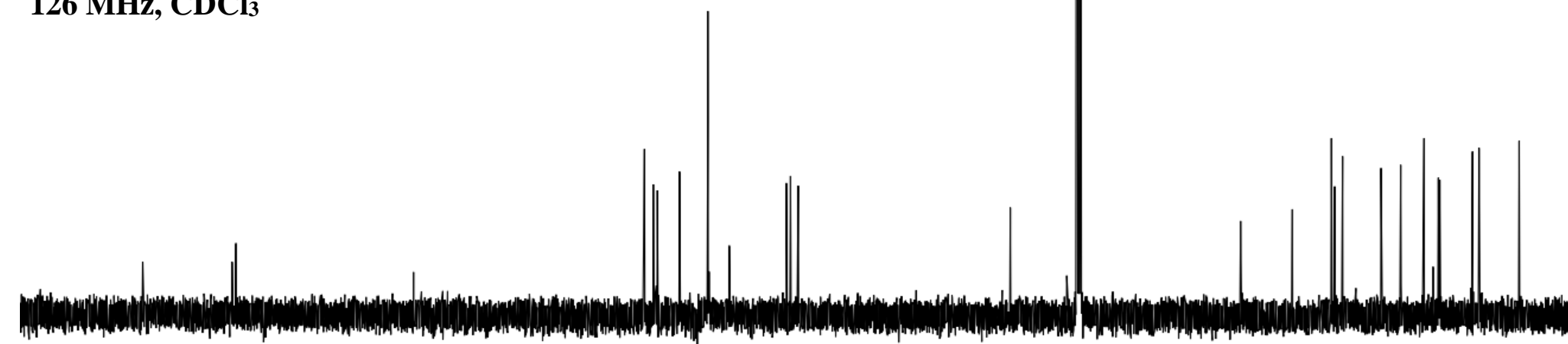

$\begin{array}{llllllllllll}220 & 210 & 200 & 190 & 180 & 170 & 160 & 150 & 140 & 130 & 120 & 110 \\ 13 \mathrm{C}(\mathrm{ppm})\end{array}$

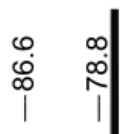

क त

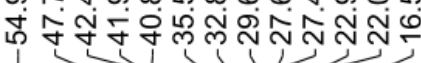

$1<1<111$

$90 \quad 80$

50

$40 \quad 30$

20

$10 \quad 0$

$0 \quad-10$ 


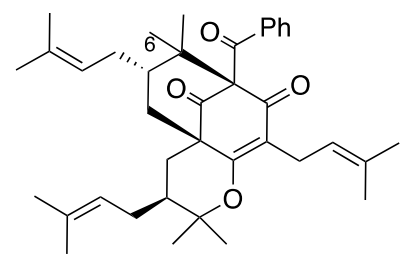

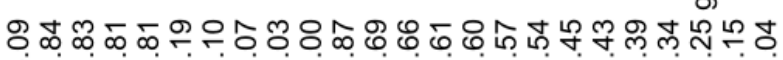

20 (-)-6-epi-garcimultiflorone A

$500 \mathrm{MHz}, \mathrm{CDCl}_{3}$

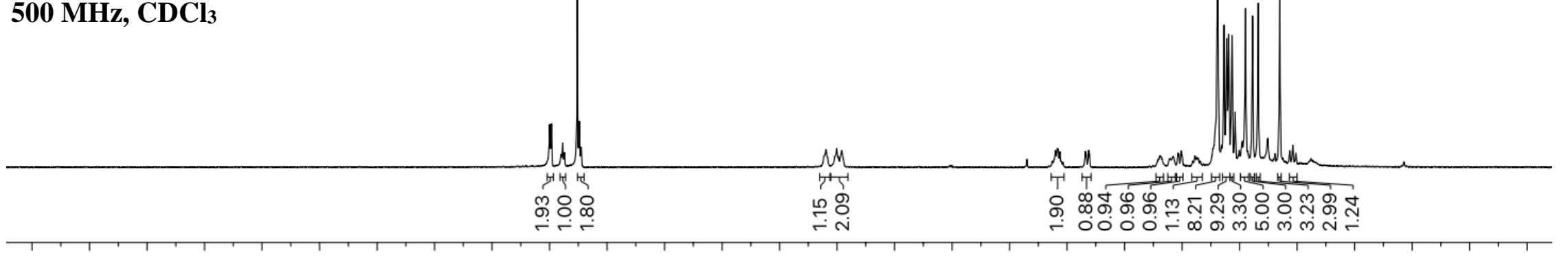

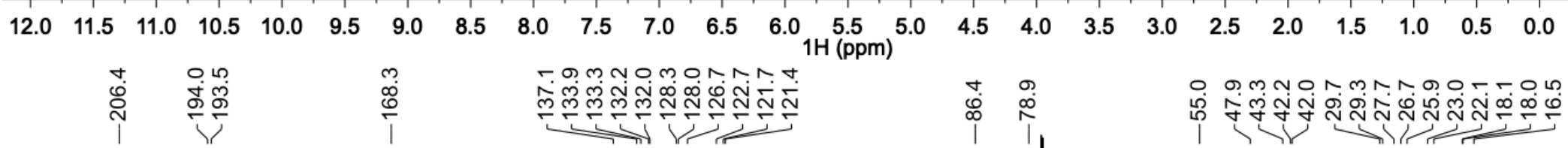

$126 \mathrm{MHz}, \mathrm{CDCl}_{3}$

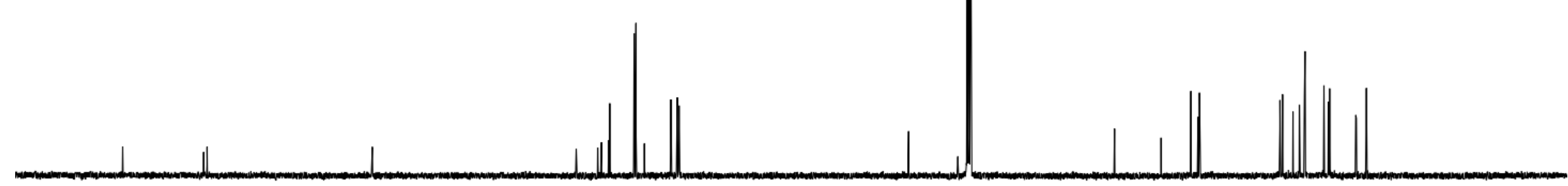

190

80

160

150

$140 \quad 130$

$\begin{array}{llr}120 & 110 & 100 \\ 13 \mathrm{C} & (\mathrm{ppm})\end{array}$

$90 \quad 80$

50

40

30

20

100

$0 \quad-10$ 
Partial gHMBC Spectrum of 20 (500/126 MHz), Key ${ }^{1} \mathrm{H}-{ }^{13} \mathrm{C}$ Correlations Highlighted

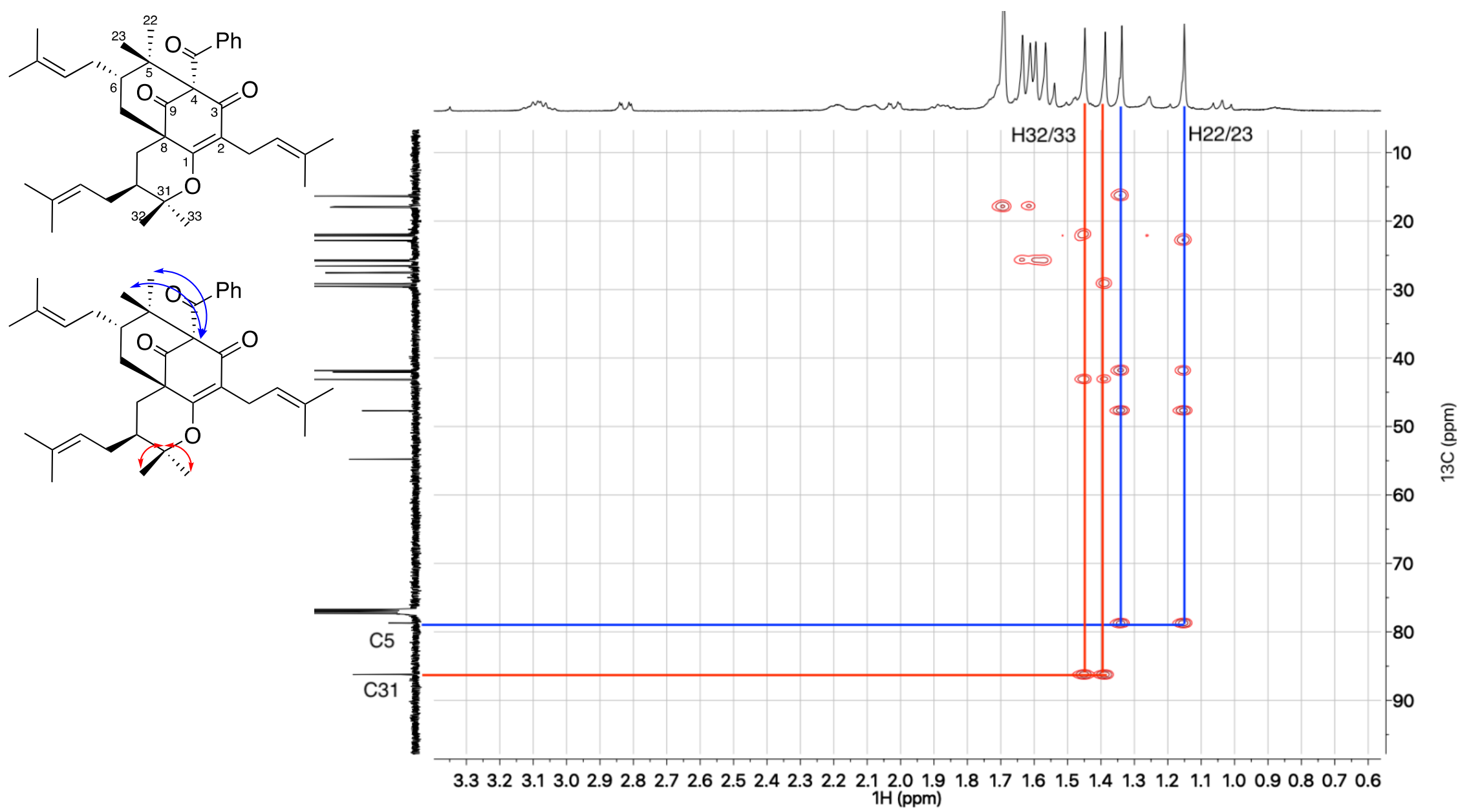



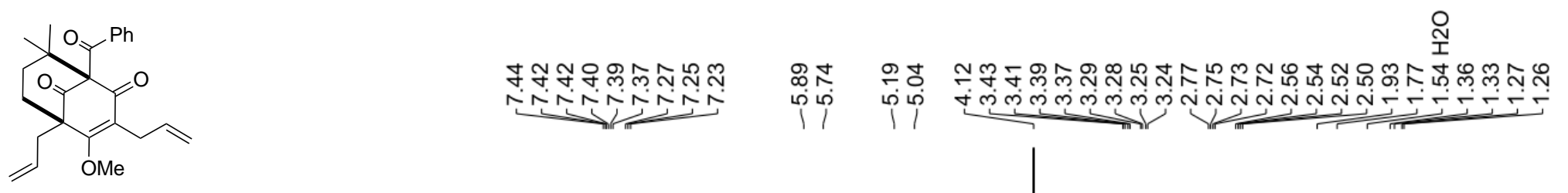

23

$400 \mathrm{MHz}, \mathrm{CDCl}_{3}$

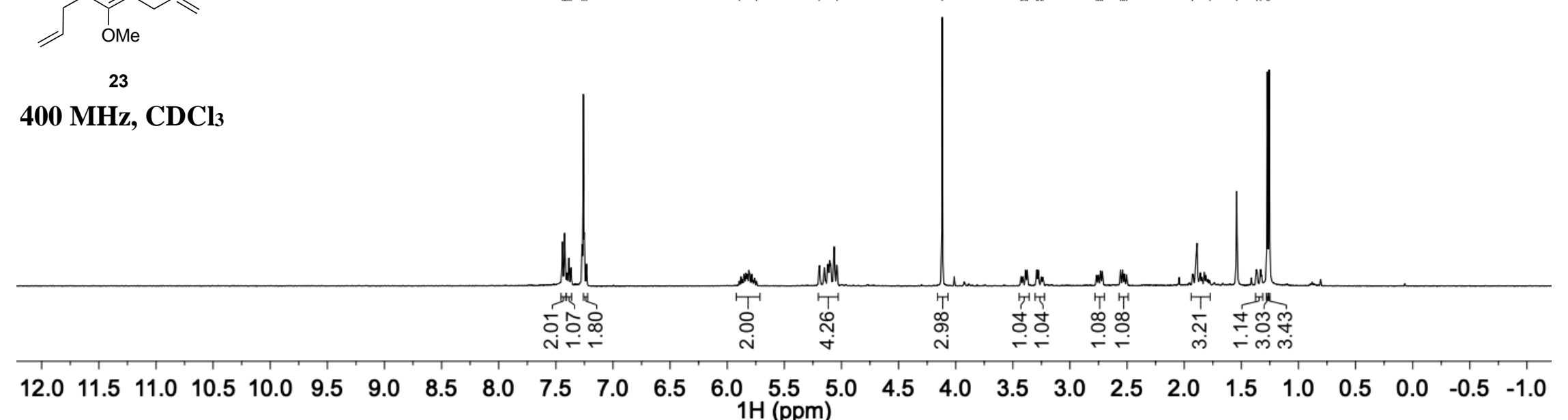

$$
\text { ஸें }
$$

$101 \mathrm{MHz}, \mathrm{CDCl}_{3}$

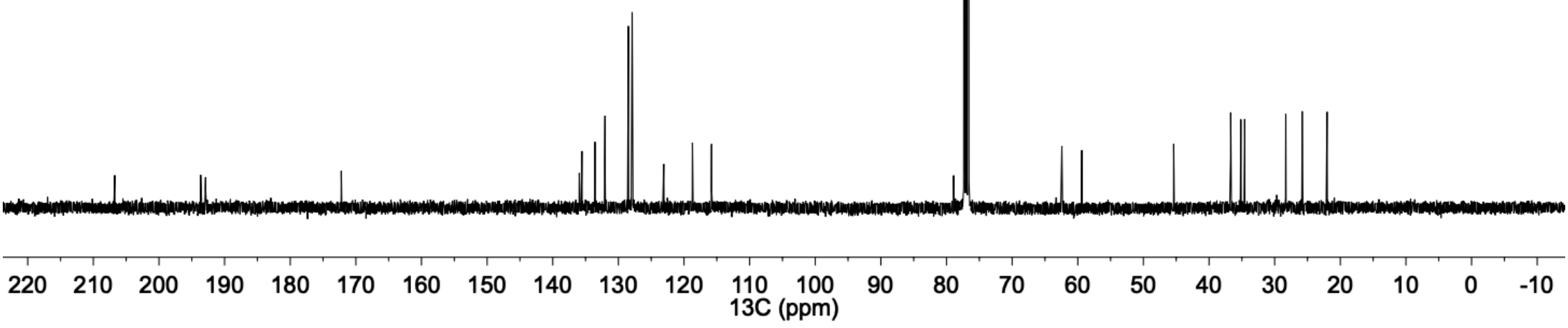




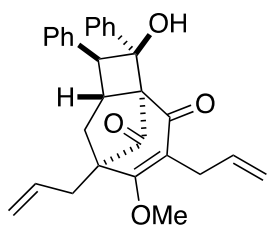

26

$500 \mathrm{MHz}, \mathrm{CDCl}_{3}$

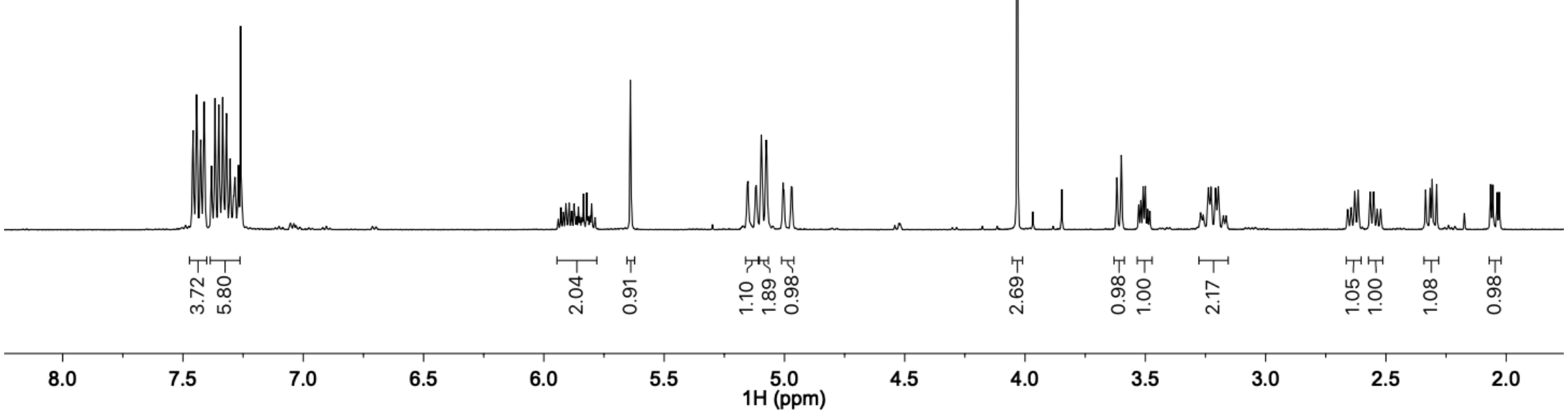




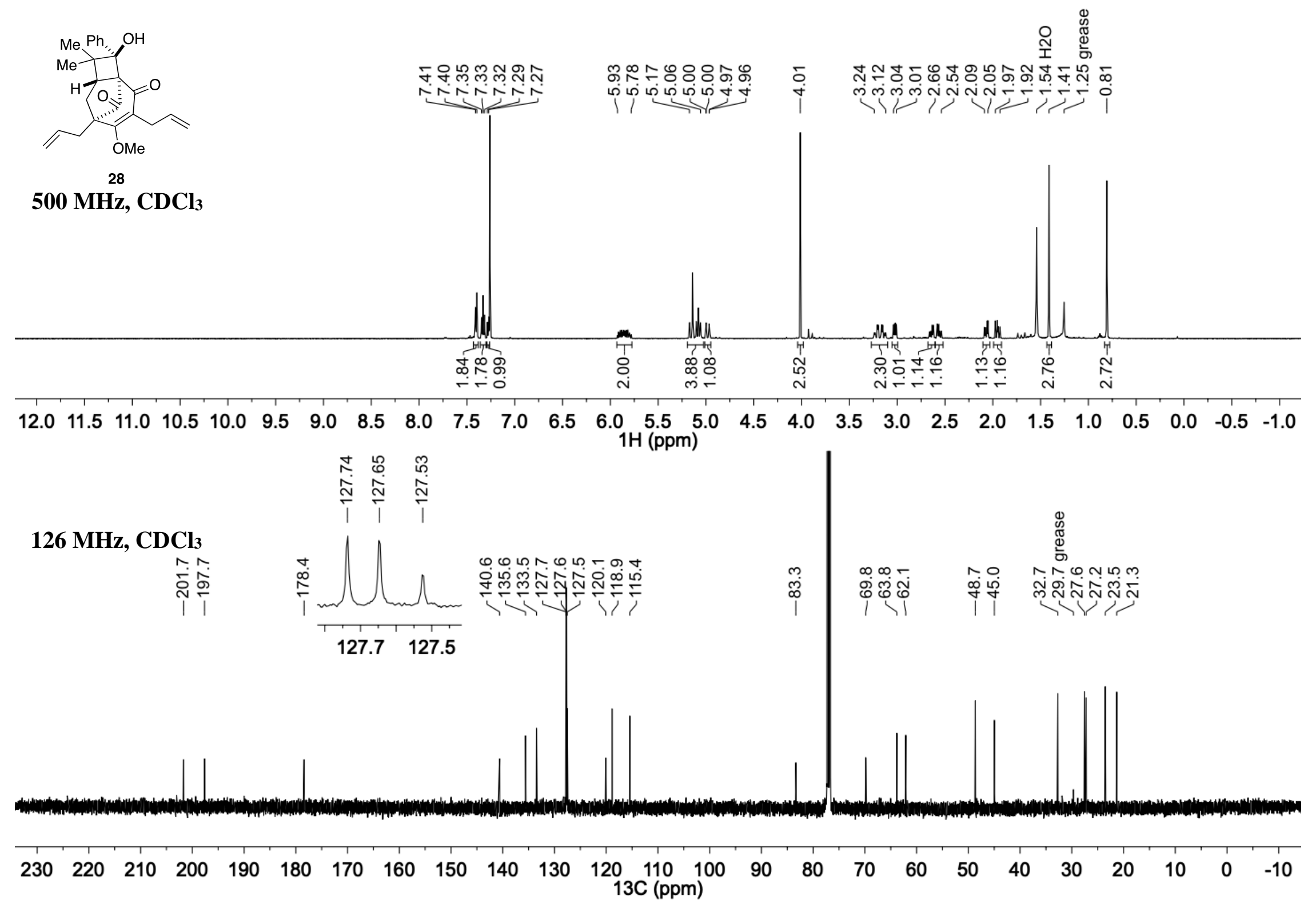


Partial gHMBC Spectrum of 28 (500/126 MHz), Key ${ }^{1} \mathrm{H}^{13} \mathrm{C}^{13}$ Correlations Highlighted

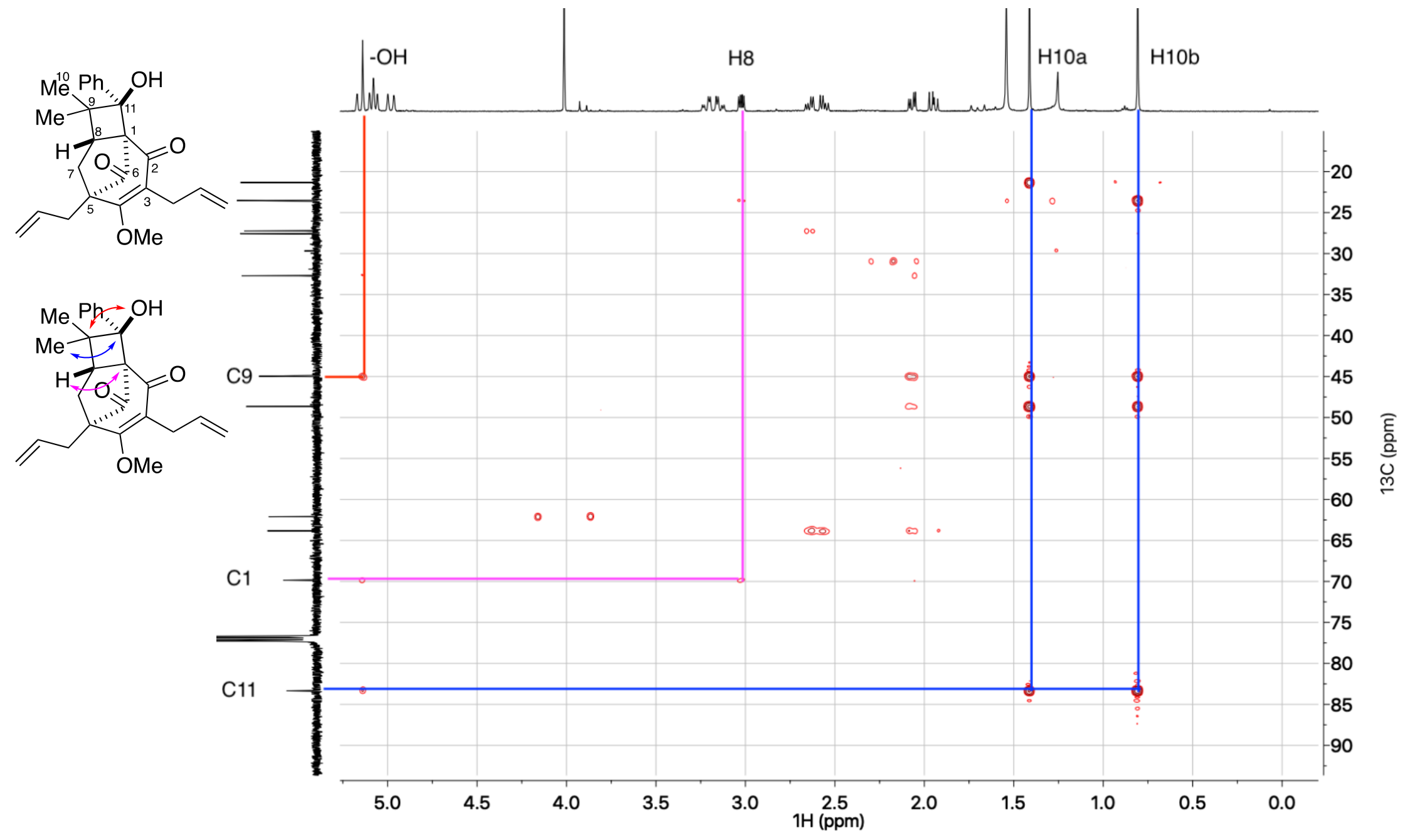




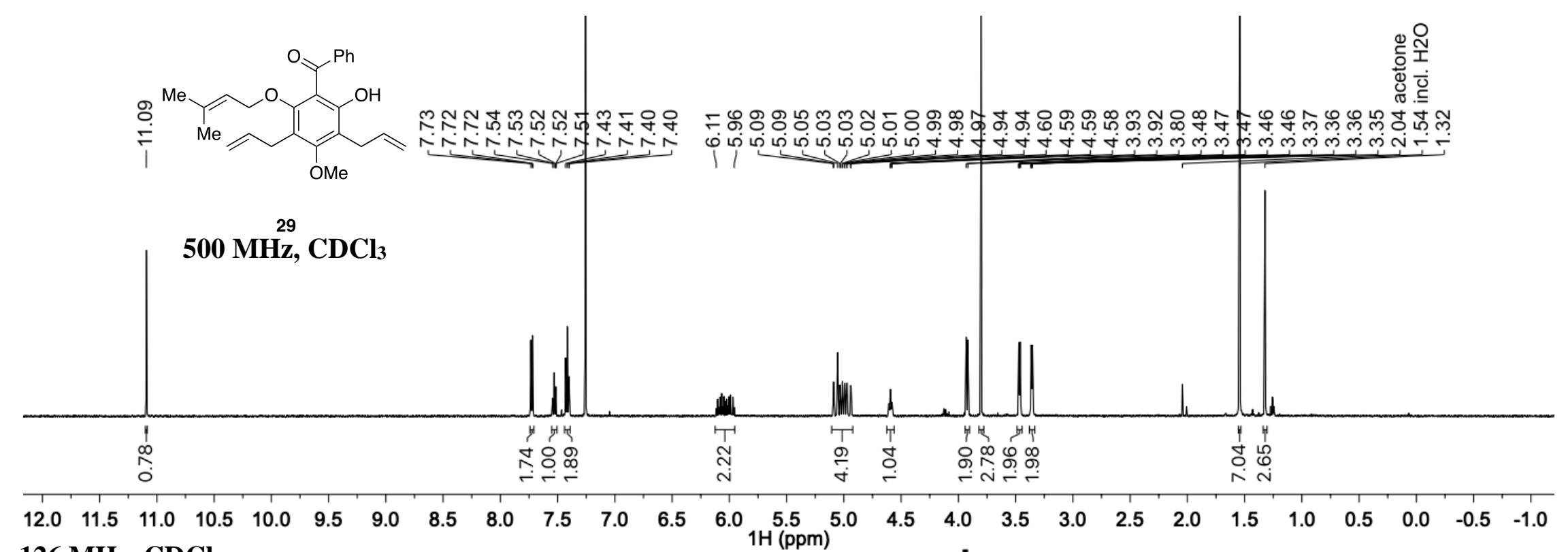

$126 \mathrm{MHz}, \mathrm{CDCl}_{3}$
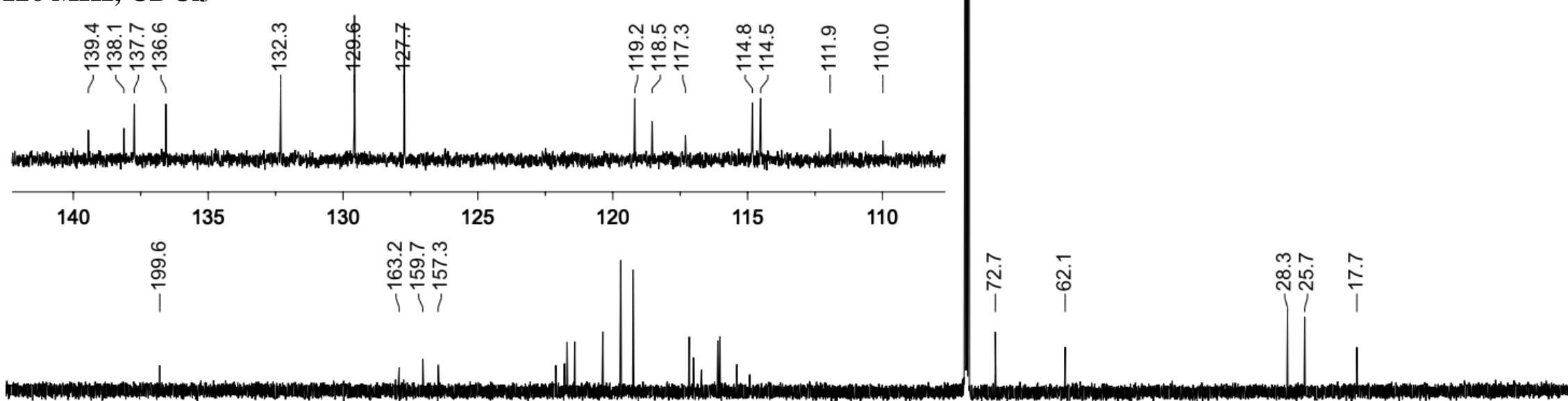

\begin{tabular}{lllllllllllllllllllllllll}
\hline 220 & 210 & 200 & 190 & 180 & 170 & 160 & 150 & 140 & 130 & 120 & 110 & 100 & 90 & 80 & 70 & 60 & 50 & 40 & 30 & 20 & 10 & 0 & -10
\end{tabular}




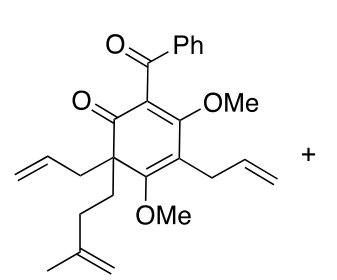

24

$400 \mathrm{MHz}, \mathrm{CDCl}_{3}$

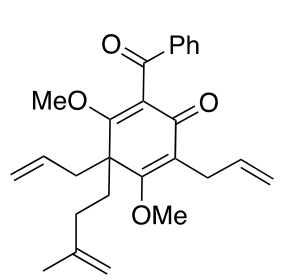

24'

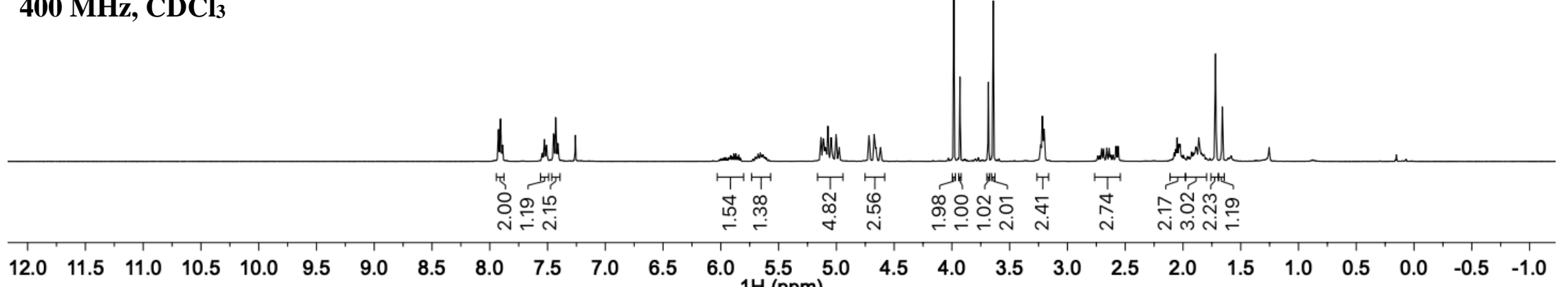

$\begin{array}{llllllllllllll}12.0 & 11.5 & 11.0 & 10.5 & 10.0 & 9.5 & 9.0 & 8.5 & 8.0 & 7.5 & 7.0 & 6.5 & 6.0 & \mathbf{1 H}(\mathrm{ppm})\end{array}$

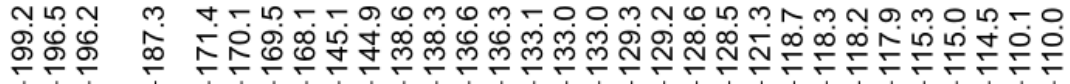

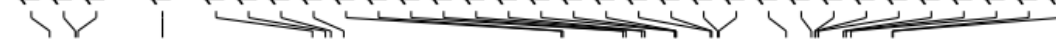

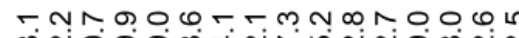

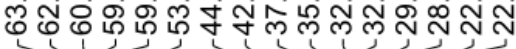

$101 \mathrm{MHz}, \mathrm{CDCl}_{3}$

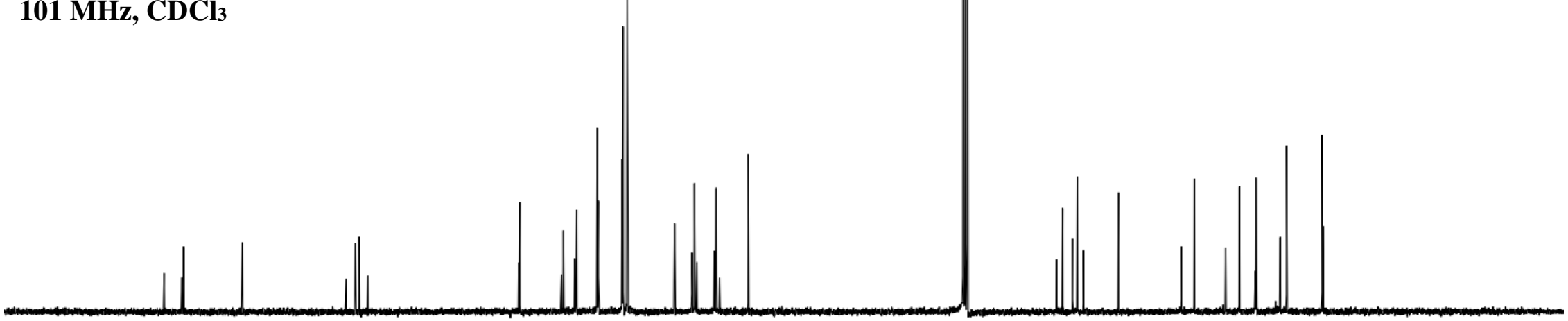

190

180

$170 \quad 160$

150

$140 \quad 130$

120

$\begin{array}{lr}110 & 100 \\ 13 \mathrm{C} & (\mathrm{ppm})\end{array}$

$\begin{array}{llll}90 & 80 & 70 & 60\end{array}$

50

30

20

100

$-10$ 